\title{
Valoriser le potentiel des mentores : recrutement, formation et soutien des mentores pour des programmes dédiés aux adolescentes
}

Miriam Temin

Population Council

Eva Roca

Follow this and additional works at: https://knowledgecommons.popcouncil.org/departments_sbsr-pgy

Part of the Family, Life Course, and Society Commons, and the Gender and Sexuality Commons How does access to this work benefit you? Let us know!

\section{Recommended Citation}

Temin, Miriam and Eva Roca. 2019. "Valoriser le potentiel des mentores : recrutement, formation et soutien des mentores pour des programmes dédiés aux adolescentes." New York: Population Council. 


\section{Valoriser le potentiel des mentores}

Recrutement, formation et soutien des mentores pour des programmes dédiés aux adolescentes

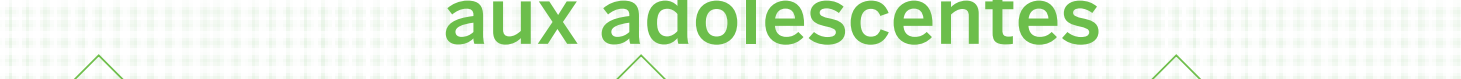


Le Population Council aide à renforcer les capacités des différents acteurs à concevoir, mettre en œuvre et évaluer des programmes efficaces et évolutifs qui visent à améliorer les conditions sanitaires, sociales, économiques et cognitives des adolescents. Nous travaillons avec des institutions allant des organisations multilatérales et bilatérales, des gouvernements aux petites organisations communautaires et réseaux nationaux.

Le Population Council s'est engagé à élargir les programmes dédiés aux filles sur le terrain, ancrée sur des données probantes, en partenariat avec des experts communautaires et avec les besoins de segmentation spécifiques centrée sur les filles.

Retrouvez plus d'informations sur les approches factuelles de la programmation pour adolescentes et des liens vers des outils et des vidéos ici : popcouncil.org/girl-centered-program-resources. 


\section{Contenu}

Remerciements

Page 2

Acronymes

3

Pourquoi les mentores sont-elles importantes pour les programmes dédiés aux adolescentes

$\begin{array}{ll}\text { Profil de la mentore, recrutement et rémunération } & 7\end{array}$

Formation de rétention des mentores

Former des groupes communautaires et offrir le contenu principal 61

Suivi et évaluation $\quad 87$

$\begin{array}{ll}\text { Annexes } & 103\end{array}$

$\begin{array}{ll}\text { Foire aux questions } & 104\end{array}$

Descriptions des programmes du Population Council 107

Liens vers les curricula complets des programmes inclus dans la 111

$\begin{array}{ll}\text { Boîte à outils de la mentore } & 113 \\ \text { Ressources et lectures supplémentaires } & \end{array}$ 


\section{Remerciements}

Cette boîte à outils de la mentore a été produite par Eva Roca et Miriam Temin, avec la contribution substantielle de Natalie Jackson et les contributions supplémentaires de Karen Austrian. Nos sincères remerciements au personnel des nombreux programmes du Population Council qui ont fourni des informations et la documentation pour sa réalisation. Les matériaux étaient à l'origine utilisés dans :

- Abriendo Oportunidades (Ouvrir des opportunités)

- Programme d'autonomisation des adolescentes (AGEP)

- Initiative pour les adolescentes - Kenya (AGI-K)

- Association bangladaise pour les compétences de vie, les revenus et les connaissances des adolescents (BALIKA)

- Biruh Tesfa (Brillant avenir)

- Filles Éveillées

- GirlsRead! (Les filles lisent)

- Nisikilize Tujengane (NISITU) (Écoutez-moi, grandissons ensemble)

- Économies sûres et intelligentes

Nos remerciements particuliers aux membres du Mentor Toolkit Advisory Group : Sigma Ainul, Sajeda Amin, Karen Austrian, Kamruzzaman Bhuiyan, Ludivine Cicolella, Annabel Erulkar, Cecilia Garces, Pamela Nyirenda, Mercy Nzioki et Silvia Tum. Nous remercions également les membres de la communauté de pratique de la programmation des adolescents ainsi que les nombreux contributeurs au développement des ressources dans cette boite à outils. 


\title{
Acronymes
}

\author{
AGI-K Initiative pour les adolescentes - Kenya \\ AJF Adolescentes et jeunes femmes \\ BALIKA Association bangladaise pour les compétences de vie, les revenus et les \\ connaissances des adolescents \\ ES Espace sûr \\ FF Formation des formateurs \\ IST Infections sexuellement transmissibles \\ FAWEZA Forum des éducatrices africaines de Zambie \\ NISITU Nisikilize Tujengane (Écoutez-moi, grandissons ensemble) \\ ONG Organisation non gouvernementale \\ PAA Programme d'autonomisation des adolescentes (AGEP) \\ SDSR Santé et droits sexuels et reproductifs \\ VBG Violence basée sur le genre \\ VIH/SIDA Virus de l'immunodéficience humaine/syndrome d'immunodéficience acquise
}




\section{Pourquoi les mentores sont-elles importantes pour les programmes dédiés aux adolescentes?}

Les programmes communautaires destinés aux adolescentes et jeunes femmes (AJF) sont de plus en plus centrés sur des clubs ou des groupes. Les programmes mis en œuvre selon cette approche, parfois appelée "espaces sûrs », font appel à des mentores pour aider les filles à développer des compétences ${ }^{1}-$ tels que des relations, des compétences et des connaissances précieuses - et pour les préparer à prendre des décisions concernant leur vie et à agir en conséquence. Des programmes effectifs ont mis les filles en contact avec des amis et des mentores, et leur ont permis d'accéder à des ressources.

Depuis des décennies, le Population Council mène et teste des programmes dans différentes régions en utilisant ce modèle. De plus en plus de preuves montrent que le développement de compétences peut accroître les chances des filles dans la vie en améliorant leur santé, leur niveau d'éducation, leurs moyens de subsistance et leur sécurité.

Les mentores sont une partie importante de nombreux programmes efficaces pour les filles, mais on dispose de peu d'informations sur les aspects pratiques du développement et du soutien d'un cadre de mentore efficace. Les perceptions sur les mentores de groupes de filles et de leurs rôles varient énormément. La définition du rôle d'une mentore dépendra des objectifs et les ambitions du programme. Certains programmes visent à fournir des compétences et des informations de base; les rôles des mentores dans de tels programmes peuvent être axés sur la création d'un environnement stimulant permettant aux participantes d'apprendre et d'appliquer le matériel. D'autres programmes s'efforcent de créer un changement de grande ampleur dans les normes communautaires concernant les filles et le genre. On peut s'attendre à ce que les mentores de ces programmes mènent un activisme et un engagement communautaire profond dans leur rôle d'agents du changement.

En réponse à l'évolution de la base de données factuelles et pour aider à éclairer les pratiques des programmes, le Population Council a développé des outils pour soutenir différents aspects des programmes de groupe pour les filles qui mettent un accent particulier sur le mentorat. De nombreuses recommandations de cette boîte à outils sont basées sur des stratégies ayant fait leurs preuves. Cependant, il convient de noter que si ces outils sont souvent utilisés dans le cadre d'études et d'évaluations, les évaluations d'impacts n'ont pas testé différentes approches de mentorat indépendamment des autres éléments du programme. Néanmoins, les aspects du mentorat décrits ici ont tous été présentés dans des programmes ayant fait l'objet d'évaluation d'impacts et se sont généralement avérés avoir des effets positifs sur les filles.

1 Les « compétences » sont les ressources humaines que les filles utilisent pour réduire les risques et élargir les opportunités. Les atouts sociaux comprennent des relations de confiance avec les autres; les atouts cognitifs comprennent les compétences en résolution de problèmes; les atouts économiques comprennent les compétences en matière de budgétisation; et les biens civiques comprennent les cartes d'identité. Une approche de création de compétences considère la fille dans son ensemble plutôt que de développer ses compétences dans un seul secteur. Pour plus d'informations : https://ssir.org/ articles/entry/how_to_give_adolescent_girls_voice_choice_and_control 
Le contenu de cette boîte à outils provient de divers programmes du Population Council. Il comprend des documents provenant de programmes de groupes communautaires destinés aux AJF âgées de 7 à 24 ans. Les ressources présentées ici ont été développées et adaptées pour une utilisation dans des pays du monde entier, notamment au Bangladesh, au Burkina Faso, en Éthiopie, au Guatemala, au Kenya, au Mexique et en Zambie; dans des contextes urbains et ruraux; et avec des filles scolarisées et non scolarisées, des filles mariées, des filles travaillant comme domestiques et autres. Des détails spécifiques sur les programmes et les liens vers les ressources sont fournis dans les annexes. Bien que ces programmes soient centrés sur les AJF qui étaient souvent mal servies par les programmes de «jeunes » dans leurs communautés, les outils peuvent également être utiles pour d'autres types de programmes, par exemple ceux qui travaillent avec des groupes de garçons et de filles qui utilisent des mentores.

Cette boîte à outils est conçue pour être pratique et conviviale pour les planificateurs de programmes, les praticiens, les formateurs des mentores et les mentores elles-mêmes. Elle comprend des informations tirées des années d'expérience du Population Council, et englobe des enseignements, des conseils et des outils spécifiques qui peuvent être adaptés et utilisés pour trouver, former, suivre, soutenir et évaluer des mentores. Dans cette boîte à outils, vous trouverez:

- Des expériences de décennies de travail du Population Council dans le domaine des programmes destinés aux adolescentes

- Des stratégies de recrutement et de formation des mentores

- Des exercices utilisés par les mentores pour animer les sessions du programme

- Des stratégies de rétention des mentores

- Des outils de suivi et d'évaluation

- Des modèles pour la durabilité

Les informations fournies dans cette boîte à outils sont regroupées en quatre catégories principales:

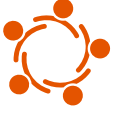

Les activités sont des actions, des présentations et des jeux qui peuvent être utilisés pendant une session de programme ou une formation de mentore.

EQ

Les études de cas décrivent comment les programmes du

Population Council utilisent les ressources des mentores.

Les guides fournissent les informations et le contexte.

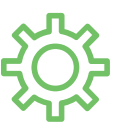

Les outills sont des formulaires, des agendas et des modèles qui peuvent être adaptés aux besoins d'un programme. 
Chaque ressource identifie également l'un des trois principaux utilisateurs : les planificateurs de programme, les superviseurs de mentore ou les mentores. Cependant, de nombreuses ressources seront applicables à plusieurs groupes d'utilisateurs. Le plus essentiel c'est que toutes les ressources contenues dans la boîte à outils sont destinées à être utilisées comme des exemples que les utilisateurs peuvent adapter au contexte dans lequel ils travaillent. Des adaptations aux besoins locaux, aux langues et aux exemples illustratifs peuvent également être justifiées. Une étape clé consiste pour les utilisateurs à comprendre la pertinence de chaque ressource, non seulement les objectifs de l'activité ou de l'outil spécifique, mais aussi la pertinence de celle-ci pour la vie des AJF.

Le but de cette boîte à outils est de partager des matériaux qui peuvent être adaptés pour répondre aux besoins de différents programmes. Nous encourageons les utilisateurs à photocopier des pages individuelles et à les modifier à la main si des modifications mineures sont nécessaires. Pour une personnalisation plus poussée, les utilisateurs peuvent copier le contenu et s'appuyer sur le document en ajoutant des logos du programme et en modifiant le texte pour répondre aux besoins des programmes spécifiques. Les fichiers modifiables des outils trouvés dans cette boîte à outils peuvent être téléchargés à : https://knowledgecommons.popcouncil.org/departments_sbsr-pgy/630/ Veuillez citer le Population Council lorsque vous utilisez l'un de ces matériaux.

Pour plus d'informations sur les approches basées sur les données probantes des programmes pour AJF et un aperçu et des liens vers des outils et des vidéos, veuillez visiter : popcouncil.org/girl-centered-program-resources. 


\section{CHAPITRE 1}

\section{Profil de la mentore, recrutement et rémunération}

$\begin{array}{llr}\text { GUIDE } & \text { Le modèle de mentore } & \text { Page } 10 \\ \text { GUIDE } & \text { Critères de sélection de la mentore } & 12 \\ \text { GUIDE } & \text { Recrutement de mentores } & 13 \\ \text { OUTIL } & \text { Annonce pour le recrutement des mentores } & 14 \\ \text { ÉTUDE DE CAS } & \begin{array}{l}\text { Programme d'autonomisation des filles adolescentes : } \\ \text { recrutement de mentores }\end{array} & 16 \\ \text { GUIDE } & \text { Recrutement : entretien et sélection } & 19 \\ \text { OUTIL } & \text { Fiche de résultat de l'entretien avec les mentores } \\ \text { OUTIL } & \text { Contrat de service de la mentore } & 20 \\ \text { GUIDE } & \text { Rémunération de la mentore } & 22 \\ & \end{array}$




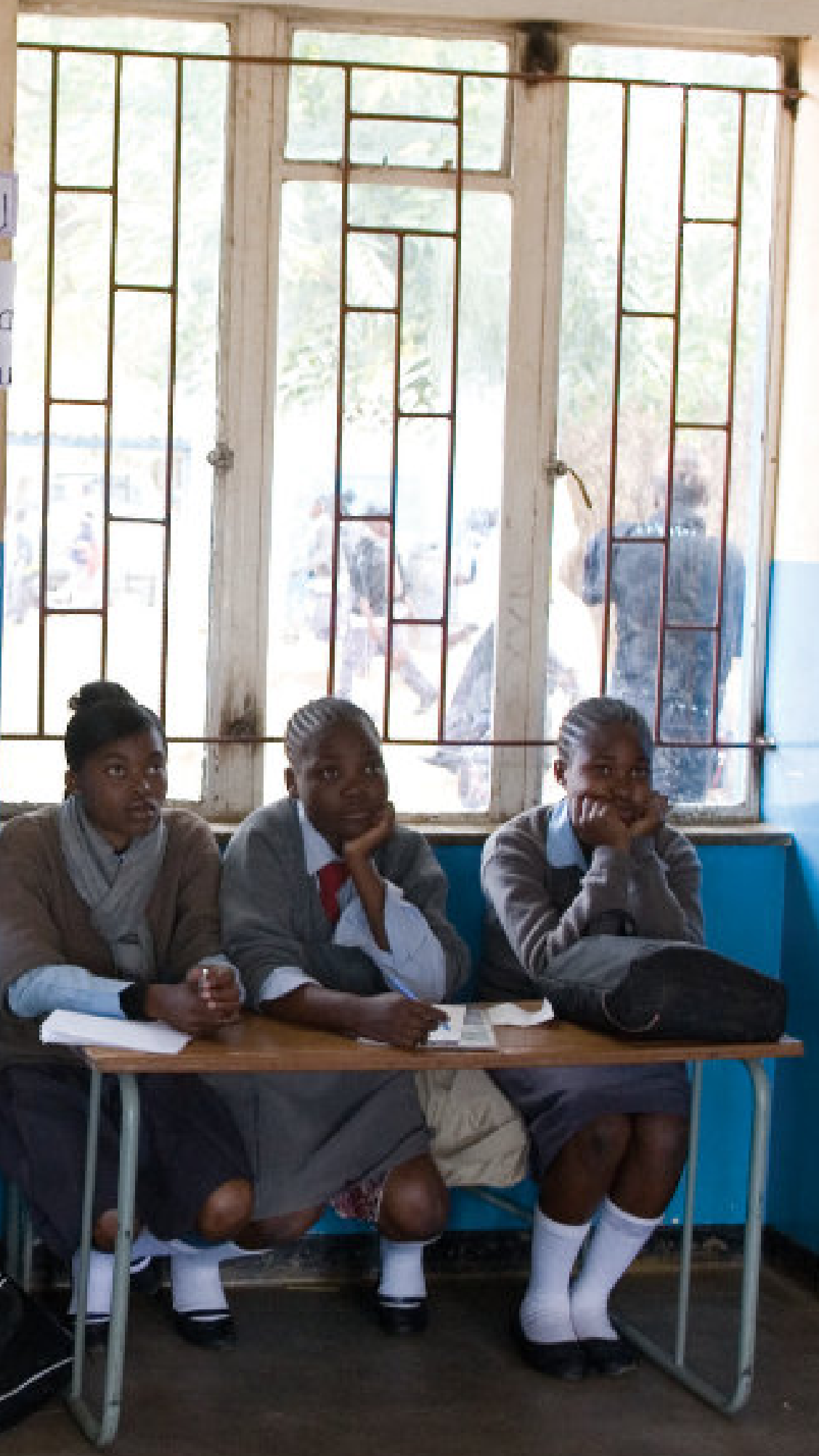

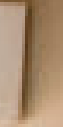


Les mentores seront généralement le principal point de contact entre le personnel du programme et les filles, leurs familles et la communauté. Les mentores peuvent être vitales lorsqu'elles répondent aux besoins critiques des filles en matière de soutien social et de modèles positifs. Les mentores ont un rôle multidimensionnel nouveau pour de nombreux programmes, et elles ont besoin de formation et de soutien.

\section{Cette section décrit les} qualités d'une bonne mentore, les principales responsabilités de la mentore, comment trouver et sélectionner des mentores, et la rémunération de la mentore

\section{LE MODĖLE DE MENTORE}

Le modèle de mentore décrit dans cette boîte à outils diffère de manière importante des modèles largement utilisés d'éducateurs pour les pairs et dirigés par des enseignants. Dans la plupart des programmes, la responsabilité principale des mentores est de transmettre le contenu de base du programme; un rôle secondaire peut être de servir de modèle et de ressource communautaire pour les filles. Les mentores peuvent également servir d'intermédiaire entre les filles dans un programme et les ressources d'une communauté, aidant à connecter les filles aux ressources communautaires. Les mentores jouent souvent un rôle dans la médiation des conflits avec les parents, les employeurs ou autres tuteurs.

Dans le modèle de la mentore, les mentores sont des femmes légèrement plus âgées que les filles du programme (par example, une mentore de 20 ans travaille avec un groupe de filles de 10 à 14 ans), alors que les pairs éducateurs ont généralement le même âge que les filles.

Les programmes d'éducation par les pairs ont tendance à élever les membres exceptionnels du groupe à un poste de leadership. En revanche, le modèle de la mentore donne aux filles plus âgées ou aux jeunes femmes défavorisées, mais à l'avenir prometteur, l'occasion de diriger. ${ }^{2}$

Les programmes dirigés par un enseignant diffèrent également. Les enseignants sont souvent beaucoup plus âgés, ont parfois un statut socio-économique plus élevé ou viennent de communautés éloignées, et ont une position d'autorité sur les filles plutôt qu'une relation qui favorise la confiance et le partage d'informations privées et sensibles. Les enseignants peuvent transmettre des informations aux filles comme si elles étaient dans une salle de classe plutôt que d'encourager la participation grâce à des méthodes interactives recommandées dans les groupes de filles.

Quelles sont les qualités d'une bonne mentore?

\section{Le choix des bonnes mentores est la clé d'une mise en œuvre réussie du programme. L'expérience montre que la caractéristique essentielle d'une bonne mentore est sa provenance. Elle doit être de la localité, être de la communauté, et vivre dans la communauté qu'elle sert. Pourquoi est-ce important?}

- Être local devrait augmenter la capacité des mentores à comprendre et à relever les défis auxquels les filles sont confrontées et permettre aux filles de s'identifier à elles.

- Être dans la communauté rend plus probable le fait que les parents et les tuteurs fassent confiance aux mentores et permettent aux filles de participer. Être de la communauté assure de l'accessibilité des mentores par les filles en cas de besoin en dehors des heures de rencontre habituelles.

2 https://knowledgecommons.popcouncil.org/departments_sbsr-pgy/583/ 
- Le recrutement local est un investissement dans le leadership féminin, permettant aux filles plus âgées et aux jeunes femmes de la localité ayant un potentiel de leadership d'acquérir et d'exercer des compétences qui seraient autrement négligées.

- II peut remettre en question les normes communautaires sur ce que les filles et les jeunes femmes peuvent faire, en fournissant des modèles de rôle alternatifs positifs pour les filles dans une communauté et en donnant finalement aux filles et aux jeunes femmes plus de " voix » dans la prise de décision au sein du ménage et de la communauté. ${ }^{3}$

Le recrutement local des mentores peut également présenter des défis. Par exemple, en tant que produits du même environnement social, les mentores peuvent défendre certaines des mêmes idées et normes auxquels le programme espère s'attaquer, en particulier autour de questions sensibles telles que la santé et droits sexuels et reproductifs (SDSR) et l'inégalité des genres. La communication ouverte, la formation et la discussion des valeurs directrices du programme sont des étapes essentielles pour équiper les mentores afin qu'elles transmettent les messages que le programme souhaite transmettre. De plus, les mentores locaux peuvent aider à transmettre ces messages d'une manière qui sera culturellement acceptable.

\section{Quelles sont les caractéristiques idéales de la mentore?}

Le profil de la mentore doit correspondre aux filles qui sont dans le programme - en d'autres termes, les mentores doivent être assez jeunes et assez semblables aux filles avec lesquelles elles travaillent pour qu'elles s'identifient à elles, mais assez âgées pour être des modèles. II est préférable que les mentores ne soient pas seulement de la même communauté que les filles du programme, mais aussi représentatives du segment des filles du programme. Par exemple, si les filles mariées sont les principales participantes, les mentores devraient également être mariées; les filles migrantes devraient être jumelées à une mentore migrante. De la même manière que les filles tirent profit du fait d'être en groupe avec des filles comme elles, elles s'épanouissent également lorsqu'elles peuvent s'identifier à leur mentore.
- Les mentores devraient être des personnes desquelles les filles pensent pouvoir apprendre. La plupart des mentores des programmes décrits dans cette boîte à outils ont entre 18 et 30 ans et satisfont aux exigences en matière d'éducation et d'alphabétisation, comme avoir terminé un certain niveau de scolarité (généralement au moins quelques années d'études secondaires). La plupart des programmes exigent que les mentores puissent lire, écrire et communiquer efficacement dans la langue locale.

- Les participantes au programme peuvent avoir leurs propres idées sur ce qui fait une bonne mentore. Par exemple, les participantes à un programme au Burkina Faso ont suggéré que les caractéristiques les plus importantes d'une bonne mentore sont d'être discrète, digne de confiance, attentionnée, polie et accueillante. ${ }^{4}$

- Ces critères sont flexibles; chaque programme doit s'adapter à la situation locale. Par exemple, les programmes peuvent avoir besoin de recourir à des mentores qui ne " correspondent » pas aux filles s'il n'y a pas assez de mentores répondant aux critères d'origine. Les mentores n'ont pas à commencer comme des candidates idéales. II peut être avantageux de former de jeunes femmes prometteuses qui n'ont pas encore eu l'occasion de diriger. En recrutant des mentores, vous pouvez offrir une chance de prospérer à des jeunes qui, autrement, sont négligées, mais qui ont le désir de travailler avec des filles. Les mentores ne doivent pas seulement être tirées du pool de personnes très performantes qui participent généralement en tant que leader.

3 https: //www.jstor.org/stable/40003326

4 https://knowledgecommons.popcouncil.org/departments_sbsr-pgy/581/ 


\section{CRITĖRES DE SÉLECTION DE LA MENTORE}

Voici un exemple de liste de qualifications qui peuvent être utilisées comme critères de sélection des mentores; la liste spécifie également ce qui est nécessaire pour être une enseignante ou une mentore communautaire, dont les rôles différaient dans le programme à l'origine de cet outil. L'outil répertorie également les étapes de sélection des mentores.

\section{Qualifications/exigences pour les mentores} enseignantes et communautaires

- Être alphabétisée/sait lire et écrire

- Être respectée dans la communauté, servir de modèle pour les jeunes femmes

- Avoir des qualités de leader

- Avoir une expérience de travail avec la communauté et les groupes vulnérables

- Avoir une expérience en matière de formation et/ou d'animation de groupes

- Avoir des connaissances de base en matière SDSR serait un atout

- Volonté et flexibilité pour répondre aux préoccupations des filles en dehors des heures d'ouverture de l'espace sûr

- Être engagée à améliorer la situation des filles dans sa communauté

- Parler couramment le français et la ou les langues locales de la communauté

- Une expérience de l'utilisation des technologies telles que les smartphones ou les tablettes est un avantage supplémentaire

\section{Critères pour les mentores enseignantes}

- Être enseignante à l'école du programme (mais pas enseignante de $5 \mathrm{e}$ )

- Avoir le niveau terminal et un certificat d'enseignement

- Avoir des qualités de leader

- Avoir une expérience de travail avec la communauté et les groupes vulnérables

- Avoir des connaissances de base en matière SDSR serait un atout
- Volonté et flexibilité pour répondre aux préoccupations des filles en dehors des heures d'ouverture de l'espace sûr

- Être engagée à améliorer la situation des filles dans sa communauté

- Une expérience de l'utilisation des technologies telles que les smartphones ou les tablettes est un avantage supplémentaire

\section{Critères pour les mentores communautaires}

- Une bénévole de sexe féminin, âgée de 20 à 40 ans résidante dans la même communauté que l'école (ou à proximité)

- Doit avoir le niveau terminal

Les étapes de sélection des mentores

- Présélectionner des mentores : 3 personnes par catégorie

- Élaborer un calendrier d'entretien avec les partenaires locaux

- Appeler les candidates pour des entretiens

- Mener les entretiens par panel de l'école (si scolaire) ou communautaire (si dans la communauté) : 2-3 membres et partenaire local

- Sélectionner les candidates

- Informer le partenaire local concerné

- Invitation à la formation (la lettre sera envoyée par le partenaire local) 


\section{RECRUTEMENT DE MENTORES}

Une fois qu'un programme a identifié les types de mentores nécessaires, comment une équipe de programme peut-elle les trouver?

Même les communautés les plus en difficulté comptent généralement quelques jeunes femmes qui ont terminé leurs études secondaires et qui ont le potentiel d'être bonne mentore.

La meilleure approche pour trouver des candidates mentores dépend du contexte et de l'endroit où les gens se rendent pour découvrir de nouvelles opportunités. Par exemple, le programme Filles Eveillées au Burkina Faso a utilisé un crieur public pour diffuser les informations sur les postes de mentore en utilisant un haut-parleur monté sur une voiture qui circulait dans les communautés concernées. Les candidates intéressées possédant les qualifications appropriées ont été invitées à soumettre des candidatures et des curricula vitae. 5

Les autres méthodes utilisées pour identifier les mentores comprennent :

- Des annonces radio ou des affiches avec l'annonce dans des endroits importants de la communauté comme les hôpitaux, les écoles et les bureaux du conseil municipal, ou distribuées lors d'événements communautaires.

- Partager la description de poste avec les principaux leaders communautaires. 6

- Recommandations par les pairs ou recrutement en utilisant les recommandations des filles ellesmêmes.

- L'outil du Population Council appelé Girl Roster $^{\mathrm{TM}} 7$ qui recueille les informations sur la population au niveau communautaire peut aider à identifier des mentores potentielles (voir boîte).

- Une fois qu'un programme est en cours d'exécution dans une communauté, les anciennes participantes peuvent obtenir un rôle de mentore, une approche appelée «leadership en cascade ».

\section{Girl Roster ${ }^{T M}$ du Population Council}

$L^{\prime}$ 'outil Girl Roster ${ }^{T M}$ du Population Council a été développé pour permettre au personnel du programme de recueillir et d'utiliser des informations sur les communautés du programme. Le Girl Roster se déroule en trois étapes :

1. Le personnel du programme visite chaque ménage dans une zone géographique donnée pour mener une brève enquête auprès d'un adulte afin de collecter des informations de base sur toutes les filles du ménage.

2. Les résultats de l'enquête sont compilés et automatiquement triés pour produire un aperçu visuel du nombre total de filles (organisé en fonction de caractéristiques importantes telles que l'âge et le statut scolaire).

3. Le personnel utilise ces aperçus pour planifier leurs programmes, en commençant par la question de savoir quelles filles recruter et quelles stratégies utilisées pour les recruter.

5 https://knowledgecommons.popcouncil.org/departments_sbsr-pgy/581/

6 https://knowledgecommons.popcouncil.org/departments_sbsr-pgy/553/

7 https://knowledgecommons.popcouncil.org/departments_sbsr-pgy/468/ 


\section{ANNONCE POUR LE RECRUTEMENT DES MENTORES}

L'annonce du recrutement des mentores doit inclure une brève description du programme, une description de poste, les qualifications et la procédure de candidature. ${ }^{8} \mathrm{La}$ description du poste est utile pour rappeler à la mentore quelles sont ses responsabilités et pour fixer les limites des attentes liées au poste. Cette annonce doit être personnalisée avec les logos des partenaires, les informations pertinentes étant ajoutées dans les espaces vides comme spécifiés.

\author{
Advertissment \\ Des mentores recherchées! \\ [Nom du programme] \\ [Nom du partenaire 1] \\ [Nom du partenaire 2$]$
} et les partenaires cherchent à recruter des

femmes bénévoles âgées de et pour travailler comme mentores pour [Nom du programme] sur une période de ans.

Le programme mettra en œuvre un programme de renforcement des compétences sociales, sanitaires et économiques pour les adolescentes vulnérables à [Pays]

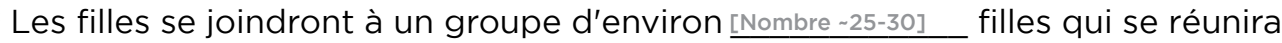
[Fréquence, généralement $1 \mathrm{X}$ par semaine] dans leur communauté, sous la direction d'une femme mentore.

Lors de leurs rencontres de groupe, les filles seront formées sur:

[Description du programme. Par exemple: éducation sanitaire et financière; établir des relations avec les filles de la communauté]

Des mentores sont recherchées pour faciliter les groupes de filles.

Les demandes doivent inclure: nom, âge, résidence/lieu, éducation, expérience de travail pertinente, numéro de téléphone.

La date limite de réception des candidatures est: [Date]

Les candidatures doivent être adressées à

Candidature de la mentore

[Nom du programme]

[Adresse] 


\section{Description de poste}

- Assister à la formation initiale des formateurs pour les mentores.

- Recruter des filles dans le programme selon des directives prédéterminées. Cela comprendra le recrutement de filles de certains âges et caractéristiques.

- Préparer et animez des rencontres hebdomadaires de groupe.

- Chaque mentore sera responsable de [Nombre] groupes, chacun avec [Nombre] filles. Les rencontres de groupe doivent être engageants, interactifs et dynamiques. Les mentores auront un ensemble de contenu de formation de base à couvrir.

- La formation comprendra des sessions sur, au minimum :

Paquet de base. Par exemple : éducation financière; santé sexuelle et reproductive; compétences de la vie; la violence aasée sur le genre

- Identifier un lieu et une heure de rencontre appropriés pour chaque rencontre de groupe, y compris d'autres lieux pour [Considérations communautaires. Par exemple: la saison des pluies.]

- Organiser des rencontres avec les parents, les conjoints et les membres de la communauté, en collaboration avec le personnel de [Nom de partenaire]

- Effectuer des visites à domicile lorsque les filles manquent plus de deux rencontres consécutifs.

- Assister [Fréquence] rencontres de mentore.

- Remplir les formulaires de rapport d'activité hebdomadaire et mensuelle du groupe

- Il est prévu que la mentore soit tenue de passer [Nombre. par exemple : 30 à 40 heures $/ \mathrm{mois}]$ de son temps.

\section{Qualifications et exigences}

- Femme âgée de et résidant dans la même communauté que les filles de son groupe

- Alphabétisée

- Respectée dans la communauté, servant de modèle aux jeunes femmes

- Possède des qualités de leadership

- Expérience de travail avec la communauté et les groupes vulnérables

- Expérience de la formation et/ou de l'animation de groupes

- Connaissance de base sur la santé sexuelle et reproductive de préférence

- Disposée à effectuer les tâches énumérées ci-dessus

- Volonté et flexibilité de répondre aux préoccupations des filles en dehors des heures d'ouverture de l'espace sûr

- Engagée à améliorer la situation des filles dans sa communauté

- Maîtrise de la langue officielle et des langues de la communauté 


\section{PROGRAMME D'AUTONOMISATION DES FILLES ADOLESCENTES : RECRUTEMENT DE MENTORES}

Le programme d'autonomisation des adolescentes (PAA) a été mené pendant deux ans pour soutenir plus de 11000 adolescentes vulnérables âgées de 10 à 19 ans dans les zones rurales et urbaines de Zambie. En tant que facilitateurs de toutes les formations destinées aux filles, il était important que les mentores du PAA aient la formation, les compétences et l'engagement nécessaires pour organiser des rencontres hebdomadaires de groupe.

Pour tenter de trouver les meilleures candidates, l'équipe du programme a élaboré une description de poste de la mentore, l'a partagée avec les principaux dirigeants communautaires et a affiché des exemplaires dans les espaces publics tels que les cliniques, les écoles et les bureaux gouvernementaux des sites du programme. Pour postuler, les candidates intéressées ont rédigé une lettre de

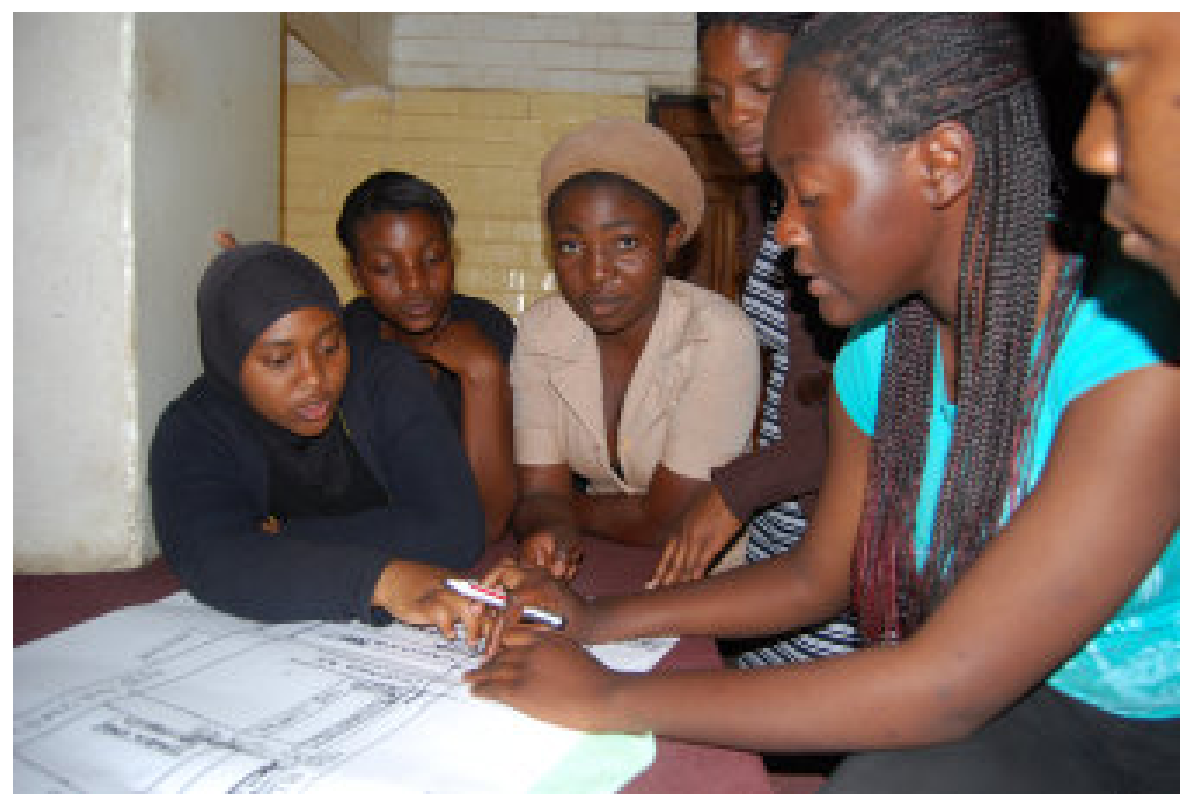

Les filles participent à des activités d'alphabétisation financière et de compétences de vie dans le cadre du programme PAA. motivation avec des détails personnels, une expérience de travail et les raisons de leur intérêt pour le poste. Lorsqu'ils étaient disponibles, elles ont joint leur curriculum vitae.

Après avoir reçu les candidatures, l'équipe du programme a préselectioné les candidates mentores et mené des entretiens professionnels pour évaluer les connaissances, les compétences, les antécédents, la motivation à travailler avec les filles et les valeurs personnelles concernant l'accès des filles aux informations et aux services de santé sexuelle et reproductive. Après avoir noté les entretiens, les 35 meilleures candidates de chaque site ont reçu une invitation à participer à une formation résidentielle des formateurs de huit jours.

Au cours de la formation, l'équipe du programme a observé les candidates en action et évalué leur performance sur les critères de sélection critiques. Après la formation, environ $70 \%$ des candidates ont été confirmées en tant que mentores PAA, $15 \%$ ont été sélectionnées comme mentores suppléantes (en cas d'abandon des mentores confirmés) et $15 \%$ n'ont pas été confirmées. Ce processus de vérification a aidé à les mentores performantes «sur papier» ou lors de

l'entretien, mais qui ont trébuché dans la pratique lors de la formation. Une fois les rencontres confirmés et fonctionnant sur une base hebdomadaire les mentores continuent de recevoir une formation et un appui technique grâce à des rencontres mensuels des mentores et une formation de recyclage ponctuelle de cing jours.

PAA a effectué une évaluation de la qualité des mentores qui a montré que les filles des groupes avec des mentores qui avaient des attitudes plus progressives sur la sexualité des adolescentes et l'utilisation de la contraception étaient moins susceptibles d'être enceintes. Cela souligne à nouveau l'importance de procéder à une évaluation minutieuse des valeurs de la mentore dans le cadre du processus de recrutement.

Pour plus d'informations, voir: Adolescent Girls Empowerment Program : Research and Evaluation Mid-Term Technical Report. 2016. https://knowledgecommons.popcouncil.org/departments_ sbsr-pgy/553/ 


\section{RECRUTEMENT : ENTRETIEN ET SÉLECTION}

En termes de recruter des mentores, c'est une bonne pratique de s'entraîner à interviewer et former plus de mentores que le programme en a besoin ; certaines mentores peuvent ne pas répondre aux attentes en matière de performances pendant la formation, et d'autres peuvent abandonner en cours de programme ou être incapables d'exercer leurs fonctions. En outre, davantage de mentores seront nécessaire si le programme se développe. ${ }^{9}$

Bien qu'il soit important d'avoir une description de poste qui précise ce que l'on attend d'une mentore, il se peut que les mentores fassent encore plus que ce que l'on attend d'elles. L'entretien avec la mentore est une bonne occasion de définir les attentes à l'égard du travail. En plus, certains programmes demandent aux mentores de réécrire leur description de poste après plusieurs mois pour mieux refléter leurs responsabilités.

Un contrat décrivant les attentes de l'organisation à l'égard de la mentore peut également être utile, tant pour la mentore que pour le staff du programme, afin de tenir la mentore responsable de ce qu'il s'est engagé à faire. Il est important de disposer d'un document auquel la mentore et le programme peuvent tous deux se référer en cas de problème (sous-performance de la mentore ou ajout de nouvelles fonctions), d'autant plus que cela peut être la première expérience de travail communautaire formel pour la plupart des mentores.

Les mentores doivent être évalués sur la base d'un ensemble de critères objectifs, et pas seulement sur la question de savoir s'elles semblent sympathiques et si elles veulent le poste (bien que ces facteurs soient également importants).

\section{Questions d'entretien des candidates mentores}

1. Pourquoi êtes-vous intéressé à devenir mentore

2. Quel âge avez-vous?

3. Où habitez-vous ? Et depuis combien de temps vivez-vous là-bas?

4. Quelle classe avez-vous fait ? Où/quelle école ? Et avez-vous d'autres formation et/ou qualifications ?

5. Avez-vous déjà fait du travail communautaire ? Si oui, quoi, où et quand?

1. Avez-vous une expérience de travail avec les adolescentes ou tout autre groupe vulnérable dans la communauté ?

2. Avez-vous de l'expérience dans la facilitation de tout type de cours sur les compétences de vie, le genre et la santé et les droits sexuels et reproductifs (SDSR) dans les écoles ou dans la communauté ? Si oui, quoi, où et quand?

3. Quels sont certains problèmes de SDSR que les filles âgées de 10 à 17 ans ne doivent pas savoir ? Demandez les raisons pour tout ce qu'elle dira.

4. Quand les adolescentes devraient-elles commencer à s'informer sur la SDSR ? Demandez les raisons.

5. Êtes-vous actuellement engagé dans un autre travail/programme?

6. Que feriez-vous si une fille de groupe de votre espace sûr venait vous voir et vous disait qu'elle est enceinte et ne peut pas poursuivre ses études?

7. Que pensez-vous qu'il faudra faire pour encourager les filles à rester plus longtemps à l'école et à terminer leurs études? 


\section{RECRUTEMENT : ENTRETIEN ET SÉLECTION (suite)}

13. Que feriez-vous si une fille de votre groupe Espace sûr n'assiste pas aux rencontres de groupe pendant deux semaines consécutives?

14. Comment évalueriez-vous vos compétences en matière de technologies? Les smartphones ou les tablettes? (Note de 0 à 3) 0 = aucune expérience, 1 = peu d'expérience, $2=$ assez expérimenté, 3 = très expérimenté. (Cette question ne sera pas pertinente pour la plupart des programmes.)

\begin{tabular}{|l|l|l|l|l|}
\hline & 0 & 1 & 2 & 3 \\
\hline NOTE & & & & \\
\hline
\end{tabular}

15. Quelle est votre disponibilité actuelle pour ce poste? Combien d'heures par semaine seriez-vous en mesure de consacrer au poste de mentore en sachant que les rencontres auront lieu les (matin/après-midi/ soir) le (jours de la semaine)?

16. Êtes-vous disponible pour assister à (nombre de jours) de formation à (lieu et date)?

17. Avez-vous des questions sur le programme? 


\section{FICHE DE RÉSULTAT DE L’ENTRETIEN AVEC LES MENTORES}

Cette fiche peut être utilisée pendant l'entretien des mentores. On peut utiliser les points pour une gamme de critères, y compris l'âge et le lieu appropriés, le niveau d'éducation et l'expérience. Des critères importants dans le contexte du programme peuvent être ajoutés. Par exemple, un programme qui attend des mentores qu'elles signalent leur présence ou d'autres informations via les téléphones mobiles peut souhaiter que les mentores soient familiarisées avec l'utilisation du téléphone portable.

Nom de la candidate

Nom de l'intervieweur

Date de l'interview

\begin{tabular}{|c|c|c|c|}
\hline Critères & Points & Résultats & Commentaires \\
\hline $\begin{array}{l}\text { Âge et emplacement appropriés : de __à } \\
\text { années; vit dans la communauté }\end{array}$ & 20 & & \\
\hline $\begin{array}{l}\text { Éducation et communication : niveau } \\
\text { d'éducation atteint; aptitude à communiquer } \\
\text { en français et/ou dans la langue locale } \\
\text { pertinente; années d'expérience pertinente }\end{array}$ & 20 & & \\
\hline $\begin{array}{l}\text { Connaissance du travail : Expérience de } \\
\text { travail communautaire; expérience de } \\
\text { l'animation de cours, que ce soit dans la } \\
\text { communauté ou dans une classe }\end{array}$ & 10 & & \\
\hline $\begin{array}{l}\text { Connaissance des compétences de vie et de } \\
\text { santé et droits sexuels et reproductifs (SDSR); } \\
\text { Expérience de travail avec des adolescentes et } \\
\text { des groupes vulnérables }\end{array}$ & 10 & & \\
\hline $\begin{array}{l}\text { Valeurs sur la santé sexuelle : niveau de } \\
\text { confort quand on parle de SDSR et de l'accès } \\
\text { des filles aux services de SDSR }\end{array}$ & 20 & & \\
\hline $\begin{array}{l}\text { Adaptabilité et perspectives sociales : tenez } \\
\text { compte de la maturité des points de vue } \\
\text { concernant les scénarios présentés, la capacité } \\
\text { à travailler de manière autonome, la flexibilité } \\
\text { des perspectives }\end{array}$ & 10 & & \\
\hline $\begin{array}{l}\text { Personnalité : tenez compte de la vigilance } \\
\text { mentale, de la première impression, des } \\
\text { manières et du comportement }\end{array}$ & 5 & & \\
\hline $\begin{array}{l}\text { Donnez à la candidate un paragraphe à lire à } \\
\text { haute voix }\end{array}$ & 5 & & \\
\hline POINTS TOTAUX & 100 & & \\
\hline
\end{tabular}

La candidate est : $\square$ Qualifiée $\square$ Non qualifiée

Observations générales :

Nom de l'intervieweur

Signature Date 


\section{CONTRAT DE SERVICE DE LA MENTORE}

Le contrat de service officialise l'accord qu'un programme conclut avec chaque mentore, en fonction de la description de poste publiée. Un contrat doit inclure des détails sur la rémunération, les heures de travail, les tâches et les pénalités pour avoir enfreint des règles spécifiques.

Contrat de travail entre : [Organisation lead]

et $\quad[$ Nom de la mentore $]$

pour [Nom du programme]

\section{CONTEXTE}

[Organisation lead]

en partenariat avec

[Organisation partenaire]

grâce au financement de [Organisation partenaire]_mettra en œuvre un programme de

[Durée] dans_[Nombre et noms des communautés] . Les fonds

permettront Nom de l'organisation engager [Nom du programme]

Les mentores seront basées dans les communautés pour diriger la mise en œuvre

des activités du programme. Les mentores du programme relèveront du

[Titre/Nom du superviseur basés dans leurs communautés respectives.

\section{DÉTAILS DU CONTRAT DE SERVICE}

Durée du contrat : ce contrat de service est conclu entre et

[Organisation lead]

et la mentore [Nom de la mentore]

pendant [Dates exactes ou début du programme et fin]

Allocation mensuelle : La mentore recevra [Fréquence; par exemple, mensuellement] une allocation de [Montant de l'allocation] pendant la durée du contrat pour couvrir les frais de transport, de communication et de déjeuner. L'allocation ne sera payée que sur la facilitation de [Fréquence; par exemple, hebdomadaire] groupes d'espaces sûrs et achèvement de [Fréquence; par exemple, mensuellement]_rapports d'activités et registres de présence des participantes à l'espace sûr.

Heures de travail : La mentore devra travailler jusqu'à [Nombre]_ heures chaque mois sur une période de [Nombre] années. Cela comprendra la gestion hebdomadaire de groupes d'espaces sûrs de [Durée de chaque rencontre] chacun et du temps supplémentaire pour les visites à domicile, les rencontres de mentore et d'autres activités de soutien.

Rapports : La mentore rendra compte au superviseur basé dans la communauté. Si, pour une raison quelconque, la mentore est incapable de s'acquitter de ses fonctions, la mentore est tenue d'en informer le superviseur.

Résiliation du contrat : L'une ou l'autre des parties peut résilier le contrat moyennant un préavis d'un mois. Dans le cas où l'une des parties ne respecte pas les conditions stipulées dans cet accord sans justification valable, l'accord peut être résilié sans préavis. 


\section{FONCTIONS SPÉCIFIQUES}

La mentore remplira les fonctions suivantes:

- Recruter des filles dans le programme selon des directives prédéterminées et en étroite collaboration avec le superviseur.

- Préparer et animer les rencontres de groupe (fréquence indiquée sur ce contrat).

- Gérer [Nombre]_ rencontres d'espace sûr par semaine, soit d'environ [Nombre; par exemple, 10-15] filles chacun.

- Faciliter rencontres de groupe interactives et dynamiques dans les espaces sûrs.

- Assurer une couverture complète et opportune du programme établi pour les espaces sûrs qui comprendra des sessions sur lesar exemple, les avantages de leducation, 'education financiere, la santé sexuelle et reproductive,

- Sécuriser et maintenir un lieu de rencontre en consultation avec le superviseur.

- Organiser des rencontres communautaires avec les parents des filles et d'autres membres de la communauté en collaboration avec le superviseur.

- Effectuer des visites à domicile lorsque les filles manquent plus de deux rencontres consécutifs en collaboration avec le superviseur.

- Assister à [Fréquence] rencontres de mentore dirigés par le superviseur.

- Participer à une formation de recyclage à la demande du superviseur.

- Compléter [La fréquence; par exemple, hebdomadaire ou mensuelle] formulaires de rapport d'activité du groupe.

- Mettre à jour les données de présence sur un base de [La fréquence; par exemple, hebdomadaire].

- Prendre la garde des ressources du programme ainsi que des documents fournis par le programme.

- Entreprendre d'autres tâches du programme qui peuvent être assignées par le superviseur.

\section{PÉNALITÉS}

Les sanctions suivantes s'appliqueront si la mentore venait à enfreindre à une des règles :

- Si une mentore manque un rencontre de son espace sûr, sans permission ou en prenant des dispositions préalables avec une collègue mentore pour couvrir le rencontre pour elle pendant son absence sera déduit [Montant d'argent] de l'allocation des mentores pour chaque rencontre manqué. L'évaluation régulière des performances sera menée chaque trimestre par [Organisation lead]

- Dans le cas où la mentore est surprise en train de falsifier ses registres de présence ou de mentir au sujet de la conduite des activités du programme, elle sera suspendue avec son allocation jusqu'à ce qu'un examen soit effectué.

\section{OBLIGATIONS}

Dans cet accord [Nom de l'orgnisation] sera limité à payer, à travers le tout compris [Fréquence; par exemple, mensuelle] une allocation de seulement

[Mécanisme, par exemple, compte bancaire]

Signé au nom de [Nom de l'orgnisation]

Directeur exécutif : Date

Mentore du programme : Date :

Témoin Date : 


\section{RÉMUNÉRATION DE LA MENTORE}

Les mentores ne sont pas des bénévoles et leur important travail doit être rémunéré de manière appropriée. En rémunérant les mentores, on augmente les chances qu'elles se consacrent au programme et qu'elles fassent un travail de qualité. Cela permet également de démontrer que le travail qu'elles accomplissent est précieux et crée une opportunité de subsistance pour les jeunes femmes de la communauté. À l'inverse, essayer d'économiser de l'argent en ne payant pas les mentores peut entraîner un taux de rotation plus élevé et augmenter les coûts de formation, ce qui réduit la durabilité du programme. Le fait de ne pas payer les mentores laisse entendre que leur travail n'est pas précieux ni valorisé. ${ }^{10,11,12}$

Les salaires varient d'un programme à l'autre en fonction du lieu et des attentes des mentores (en termes de temps consacré et de tâches); toutefois, les programmes devraient toujours payer aux mentores un montant similaire aux salaires pratiqués dans la communauté pour un travail comparable, tel que les relais gouvernementaux ou les agents de santé communautaire. La gamme des allocations que les programmes du Population Council ont donné récemment se situe entre 40 et 150 dollars américains par mois pour près de 40 heures de travail. Dans I'ensemble des programmes étudiés dans le cadre de la préparation de cette boîte à outils, les mentores dans le cadre d'un programme spécifique ont été payées à des montants similaire, bien que dans certains cas elles aient reçu des primes pour la gestion de groupes supplémentaires. Certains programmes prévoient une compensation supplémentaire, telle que prêt de vélos, paiement des transports, déjeuner, les bottes et imperméables pour se protéger de la pluie, primes de vacances, crédit téléphonique et des forfaits internet pour les téléphones, et des augmentations de salaire pour chaque année de mentorat (par exemple, une augmentation de 5 à $10 \%)$.
Le paiement lié à l'accomplissement des responsabilités énoncées dans le contrat de la mentore est important, mais des contrats détaillés peuvent également entraîner des plaintes de la part des mentores lorsqu'on leur demande d'effectuer des tâches qui ne relèvent pas de leurs responsabilités habituelles. Trouver un équilibre entre le fait d'avoir des attentes claires quant aux responsabilités des mentores tout en ne limitant pas trop les petites tâches supplémentaires qui peuvent les aider à accomplir leur travail sans exiger une rémunération plus élevée peut être un défi qu'il vaut la peine d'aborder à l'avance. Certains programmes comportent des dispositions visant à réduire la rémunération des mentores si elles manquent des séances ou falsifient des dossiers, bien que cela soit difficile à appliquer dans la pratique. ${ }^{13}$

Les mentores apprécient également les avantages non financiers, tels que les certificats obtenus à l'issue de la formation de mentore, l'expérience de mentorat, l'acquisition de nouvelles compétences et la reconnaissance ou les récompenses pour un engagement ou des performances exceptionnels. Par exemple, le programme Abriendo Oportunidades au Guatemala donne aux mentores la possibilité de participer à des formations spéciales sur des sujets d'intérêt, tels que les droits de I'homme et la recherche sociale. ${ }^{14}$ Le statut social de la mentore dans la communauté peut également être une motivation pour les mentores.

Les modes de rémunération des mentores diffèrent d'un programme à l'autre. La plupart des programmes exigent des mentores qu'elles ouvrent un compte bancaire (pour de nombreuses mentores, c'est leur premier compte) pour qu'on y dépose leurs allocations mensuelles après avoir remis leur rapport mensuel. Les programmes peuvent également envisager des transferts d'argent par téléphone portable pour payer les mentores lorsqu'elles sont disponibles. Les paiements en espèces sont déconseillés car pouvant susciter des risques de vol.

\footnotetext{
${ }^{10}$ https://knowledgecommons.popcouncil.org/departments_sbsr-pgy/578/

${ }^{11}$ https://knowledgecommons.popcouncil.org/departments_sbsr-pgy/583/

12 https://knowledgecommons.popcouncil.org/departments_sbsr-pgy/579/

${ }_{13} \mathrm{https}$ ///knowledgecommons.popcouncil.org/departments_sbsr-pgy/552/

${ }^{14}$ https://youtu.be/t1hexSWHguA
} 


\section{CHAPITRE 2}

\section{Formation et rétention des mentores}

ÉTUDE DE CAS GirlsRead! : Formation de mentore et rencontres mensuels

OUTIL

OUTIL

OUTIL

ACTIVITÉ

ACTIVITÉ

ACTIVITÉ

ACTIVITÉ

ACTIVITÉ

ACTIVITÉ

ACTIVITÉ

OUTIL

ACTIVITÉ

ACTIVITÉ

ÉTUDE DE CAS
Agenda de la formation des formateurs : Faciliter les groupes et l'engagement communautaire

Formulaire des évaluations quotidiennes

Programmes des rencontres mensuels des mentores

Cartes « Neuf Actes»

Compétences en facilitation

Méthodologies de formation

Le rôle de la mentore dans l'enseignement et l'apprentissage

Présentation de la formation : Introduction au counseling

Session de formation des mentores sur la VBG et les droits humains

Session de formation des mentores sur le travail avec les filles vivant avec un handicap

Programme de formation de recyclage des formateurs

Formation de recyclage des mentores

Cérémonie de clôture de la formation des mentores 

seatsie t)

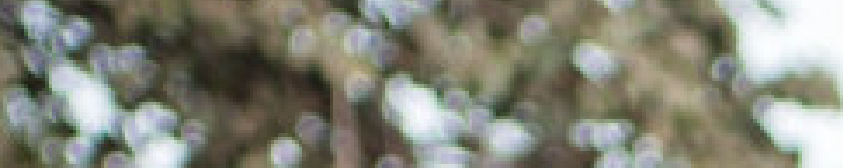
aribute: 85 की

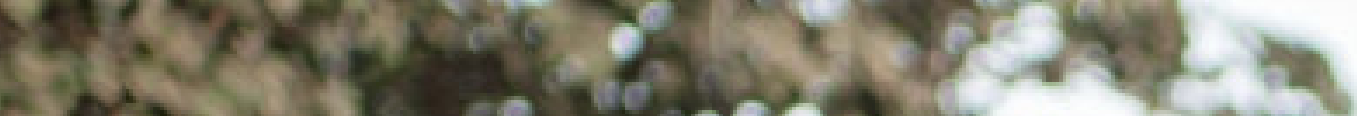

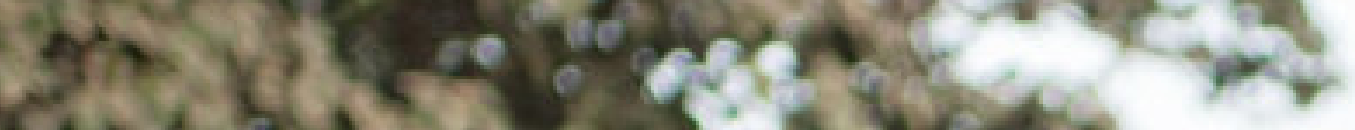
vinthasa 5 was

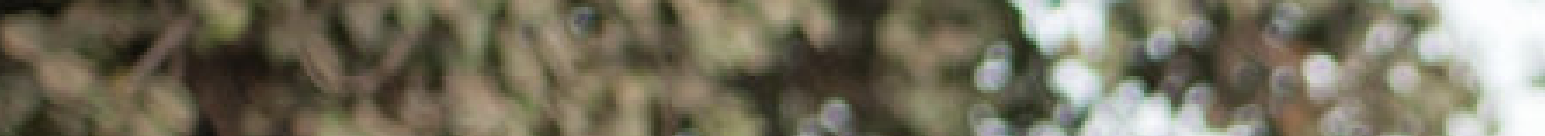

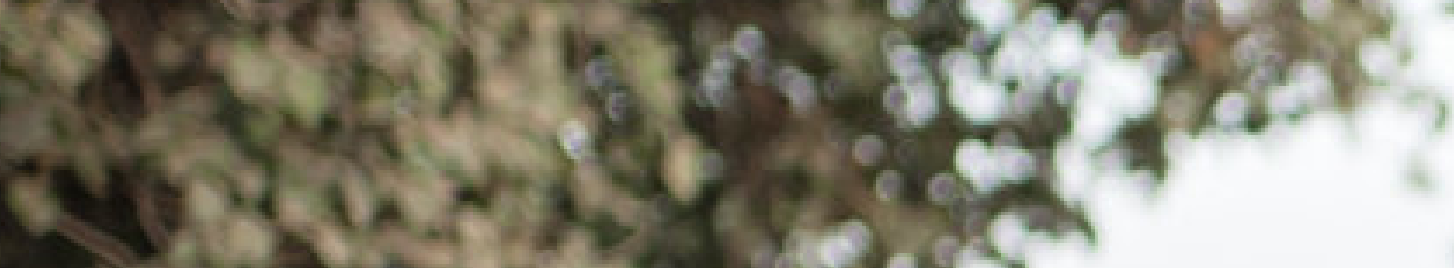

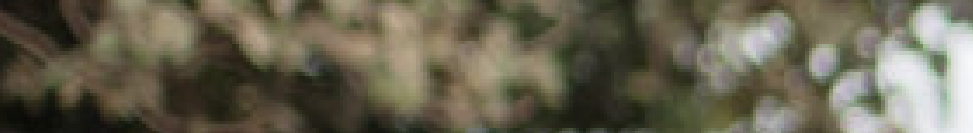

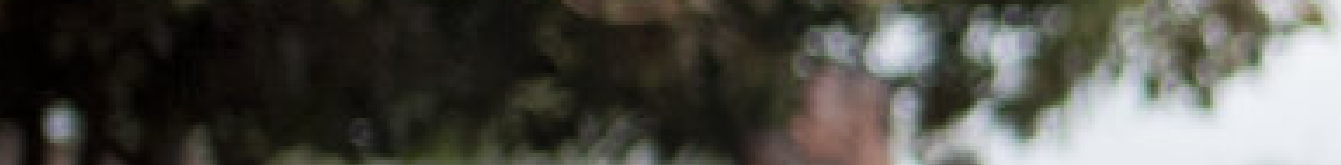

$$
20
$$

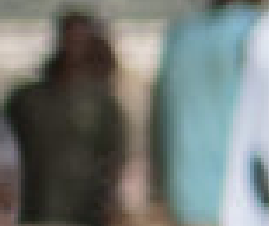

1 ta 0

$\mathrm{Sat}$ 1 1)
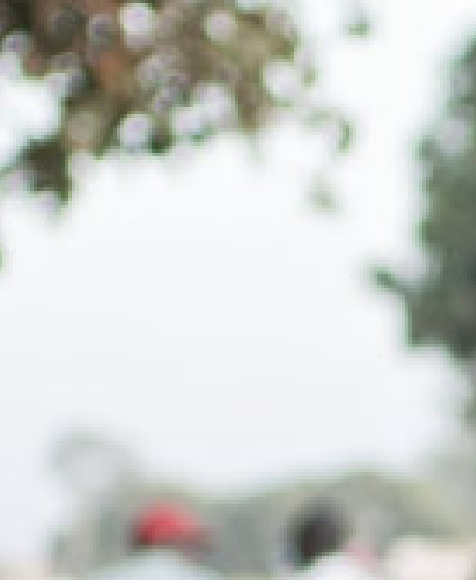

7.

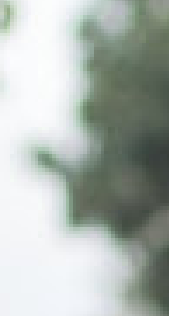




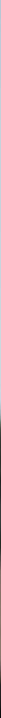

$\min _{4}$

$6-1)$

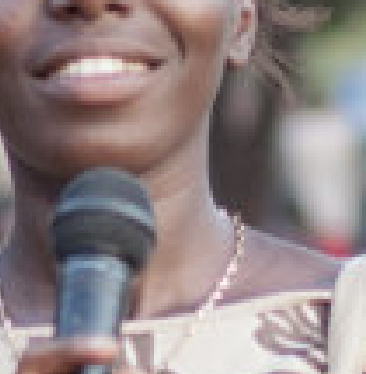

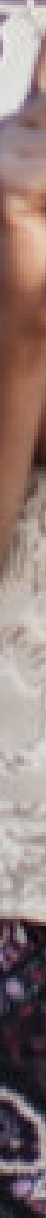

(2)

का 51

rame (c)
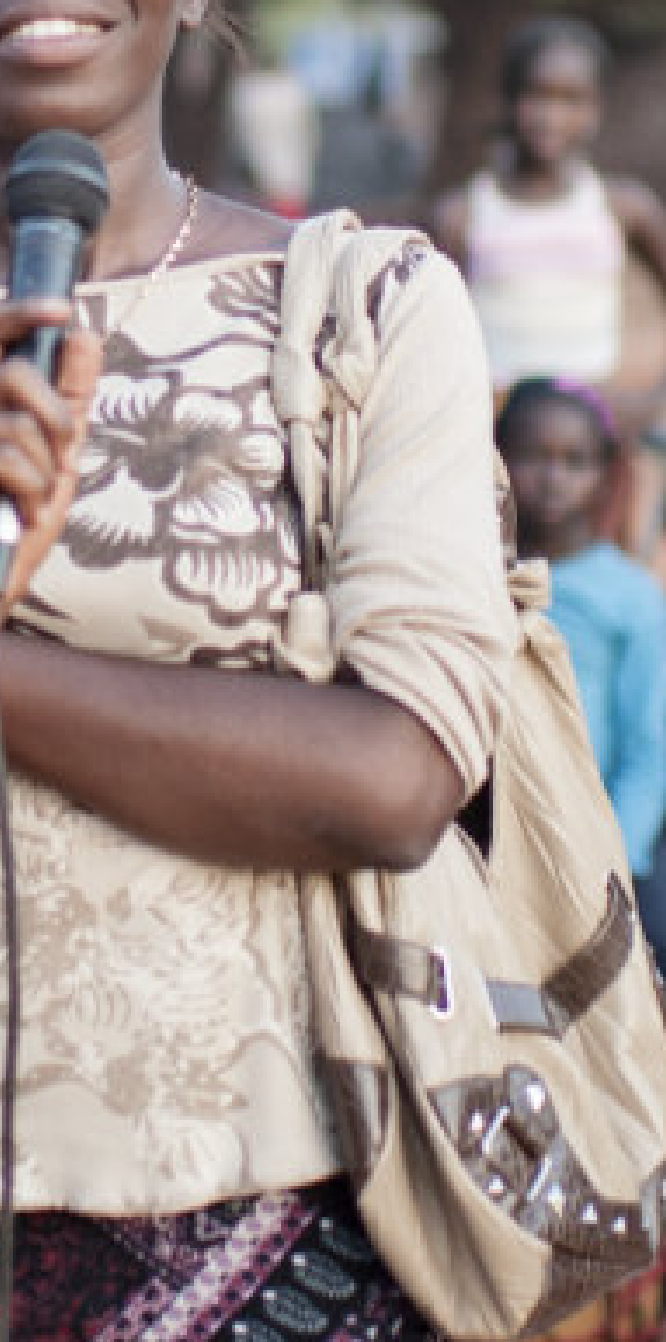

4.

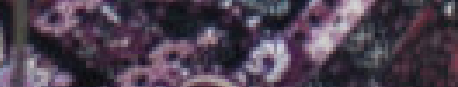

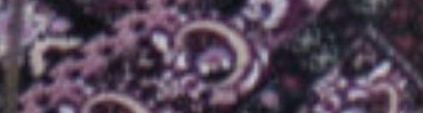


Une formation adéquate-et un recyclage-des mentores pour remplir leurs rôles est vitale pour la réussite d'un programme. Les mentores reçoivent une formation sur les programmes qu'elles mettront en œuvre, ainsi que sur la façon de jouer leur rôle en tant que mentore. Les mentores doivent être à l'aise avec le matériel pédagogique du programme et pratiquer de nouvelles techniques d'enseignement et de facilitation. On s'attend à ce que de nombreuses mentores en formation enseignent une matière qui ne leur est pas familière, leur formation est donc l'occasion de leur transmettre des valeurs clés, notamment autour des sujets sensibles comme la santé sexuelle et reproductive.

La formation n'est pas un événement ponctuel; les mentores ont besoin d'un accompagnement, d'un encadrement et d'occasions d'apprentissage continu. La plupart des programmes offrent des formations de recyclage au fur et à mesure de la progression du programme, et certains ont des rencontres hebdomadaires ou mensuels avec le personnel du programme afin que les mentores puissent poser des questions, partager leurs expériences, obtenir du soutien et mettre en pratique de nouvelles compétences.

Les mentores peuvent s'aider mutuellement à s'épanouir lorsqu'elles disposent d'un espace pour partager régulièrement les défis et les succès. Par exemple, en donnant aux mentores l'occasion de se réunir et de s'exercer à animer des séances entre eux, on renforce le capital social et on les aide à se motiver, à apprendre les unes des autres et à se soutenir mutuellement. Cela les aide également à construire leurs réseaux sociaux. ${ }^{15}$

La section suivante fournit des exemples de la façon dont les programmes du Population Council ont géré les formations. Sont inclus des exemples d'agendas pour des formations de différentes durées, ainsi que des présentations et des activités utilisées.

${ }^{15}$ https://knowledgecommons.popcouncil.org/departments_sbsr-pgy/583/ 


\section{GIRLSREAD! : FORMATION DE MENTORE ET RENCONTRES MENSUELS}

GirlsRead! La Zambie a testé l'utilisation de la technologie de la lecture électronique pour améliorer l'alphabétisation et l'action des filles par des groupes de filles dirigés par des femmes mentores, et l'engagement communautaire des parents, des administrateurs scolaires et des dirigeants communautaires; et à travers ces voies, contribuer à une réduction du risque de VIH. Le programme a été mis en œuvre avec des filles de 7e sur trois sites en Zambie, dans 36 écoles publiques, touchant plus de 2000 filles sur une période de deux ans. Le programme GirlsRead! a utilisé des paires de mentores (une mentore communautaire et une mentore enseignante) dans chaque école pour diriger les rencontres hebdomadaires dans les espaces sûrs.

La formation et le soutien continu des mentores de GirlsRead! étaient essentiels au succès du programme. Après avoir subi un processus de recrutement rigoureux, les candidates sélectionnées ont suivi une formation résidentielle des formateurs de six jours où elles ont appris la conception et la raison d'être du programme, leur rôle en tant que mentores de GirlsRead!, les thèmes du programme d'autonomisation, les techniques de facilitation non formelles et interactives, des exercices de clarification de valeur relatifs aux droits des filles d'accéder aux informations et services de santé et droits sexuels et reproductifs (SDSR), et comment utiliser le lecteur électronique et animer les sessions du programme de lecture électronique.

La formation s'est axée sur le renforcement de capacité technique des mentores de GirlsRead! à animer efficacement des sessions hebdomadaires (en abordant à la fois les composantes de l'autonomisation et de la lecture électronique du programme) tout en leur enseignant des outils et des techniques pour établir des relations de confiance avec les filles, les parents, les membres de la communauté et les administrateurs scolaires. Les séances pratiques étaient également au cœur de la formation, avec des mentores facilitant le programme GirlsRead! devant les pairs et l'équipe du programme, suivi d'une session de feedback constructif. L'expérience de la formation des formateurs a jeté les bases d'un environnement sûr et favorable pour que les mentores apprennent et s'épanouissent pendant la durée du programme.

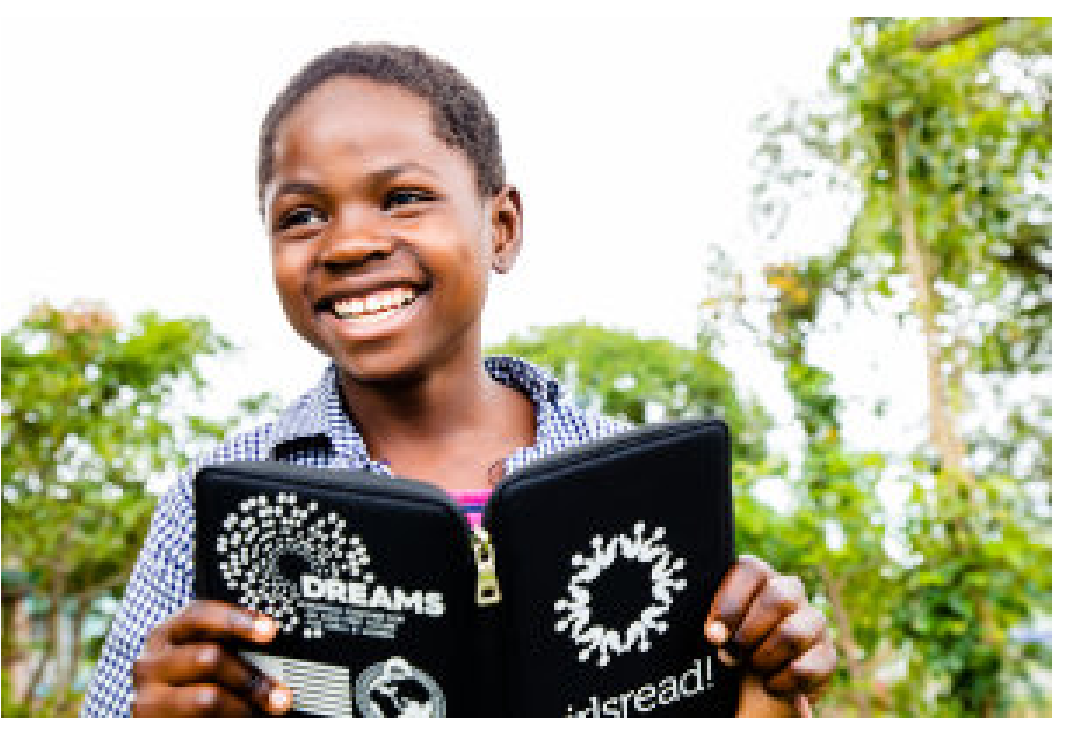

L'objectif général de GirlsRead! est d'améliorer l'apprentissage et d'accélérer la progression vers l'école secondaire des adolescentes zambiennes.

Après la formation des formateurs, les mentores sont retournées dans leur communauté et ont commencé à exécuter chaque semaine des sessions de GirlsRead!. A la fin de chaque mois, les mentores se sont réunies avec l'équipe du programme au niveau du district pour partager les mises à jour du mois précédent, y compris les succès, les défis et les solutions proposées. Les mentores ont également soumis des rapports d'activités et ont reçu des allocations mensuelles à ce moment-là, ce qui a motivé une participation régulière. Au-delà de la gestion du programme et des volets administratifs, ces rencontres ont permis aux mentores de recevoir : une assistance technique continue et une formation sur des sujets critiques du programme d'autonomisation; des conseils sur la façon de résoudre les problèmes liés aux lecteurs électroniques et à l'application de saisie de données électroniques; et des conseils sur la manière de gérer les renvois et de signaler les problèmes émergents des VBG ou de SDSR. Les rencontres mensuels des mentores ont élargi les connaissances et les compétences des mentores, fourni un soutien technique et émotionnel et amélioré le suivi des performances des mentores audelà de la formation des formateurs initiale et des visites de suivi régulières du personnel du programme.

Pour plus d'informations, voir : GirlsRead! Zambie : DREAMS Innovation Challenge. 2018. Bref. https: // knowledgecommons.popcouncil. org / départements_sbsrpgy/614/ 


\section{LES ROUAGES DE LA FORMATION}

Au début d'un programme, une formation initiale de mentore est généralement effectuée. Cette formation peut aller de quatre jours à deux semaines et comprend généralement le curriculum à dispenser, des sujets tels que les compétences de gestion de groupe et la discussion sur les responsabilités de la mentore, comme les visites à domicile ou les rencontres communautaires.

Les programmes qui exigent que les mentores utilisent des outils tels que les téléphones portables ou les tablettes forment également les mentores à ces compétences pour le moment. Les mentores de BALIKA (Association bangladaise pour les compétences, les revenus et les connaissances des adolescents), ${ }^{16}$ par exemple, ont reçu une formation à l'entretien des téléphones portables, à la photographie et aux premiers secours. Les formations doivent être interactives et offrir aux mentores la possibilité de jouer des rôles et de mettre en pratique les compétences qu'elles utiliseront dans le cadre du programme.

La durée appropriée de la formation initiale sera déterminée par la quantité et le type de matériel pédagogique que les mentores sont censées maîtriser, et par le niveau de familiarité des mentores avec le contenu et les approches. Par exemple, le programme BALIKA comprenait quatre jours de formation sur les compétences de base de la vie courante et trois jours sur les questions de genre. ${ }^{17,} 18$

Dans le cadre du programme Filles Eveillées au Burkina Faso, la formation a d'abord duré trois jours. Puis les mentores ont demandé plus de temps pour une discussion approfondie du programme et des techniques d'animation, de ce fait les formations ont été étendues à quatre jours. ${ }^{19}$

Les possibilités pour les mentores d'apprendre et de mettre en pratique leurs compétences suivent souvent une formation initiale. Ces opportunités ont généralement lieu lors des formations de recyclage organisées deux à trois mois après le début d'un programme, et lors de rencontres hebdomadaires ou mensuels avec les coordinateurs de site. Au cours de ces rencontres mensuels ou hebdomadaires, les mentores soumettent leurs données de participation, discutent des succès et des défis, et suivent de brèves formations en rapport avec les sessions à venir. ${ }^{20}$

Un programme pré-établi peut aider le personnel du programme à structurer une formation de mentore.

\footnotetext{
${ }^{16}$ https://www.popcouncil.org/balika

${ }^{17}$ Les sujets relatifs au genre comprenaient la différence entre le sexe et le genre, les normes de genre, l'égalité des genres, la discrimination entre les sexes, la violence basée sur le genre, et les conséquences du mariage des enfants.

$18 \mathrm{https} / / /$ knowledgecommons.popcouncil.org/departments_sbsr-pgy/557/

19 https://knowledgecommons.popcouncil.org/departments_sbsr-pgy/581/

${ }^{20} \mathrm{https} / / /$ knowledgecommons.popcouncil.org/departments_sbsr-pgy/552/
} 


\section{AGENDA DE LA FORMATION DES FORMATEURS : FACILITER LES GROUPES ET L’ENGAGEMENT COMMUNAUTAIRE}

Cet exemple d'agenda d'une formation de formateurs (FF) de 10 jours fournit un exemple différent de la façon d'organiser un atelier d'introduction légèrement plus long. II se concentre davantage sur l'engagement communautaire parce qu'il provient d'un programme communautaire plutôt que scolaire.

Cet agenda comprend du temps pour discuter de la recherche qui a été menée dans le cadre du projet. Les objectifs de l'atelier étaient de permettre aux participantes d'acquérir une compréhension globale des concepts du programme, d'obtenir des informations précises sur les programmes, et d'acquérir et de pratiquer des compétences dans les méthodes participatives à utiliser dans les sessions de l'espace sûr.

\section{Objectifs :}

L'objectif général de la formation est de renforcer la capacité des mentores à animer des groupes d'espaces sûrs et de dispenser efficacement des curriculums sur la santé, des compétences de vie et d'éducation financière aux filles participant au programme. Les objectifs spécifiques de la formation sont de permettre aux participantes de :

- Acquérir une compréhension approfondie des concepts du programme.

- Acquérir des informations précises sur le programme.

- Acquérir des compétences pratiques pour faciliter une gamme de méthodologies interactives à utiliser pendant les sessions d'espace sûr.

Résultat attendu : des mentores confiantes et compétentes capables de mettre en œuvre des activités de l'espace sûr et animer des sessions sur la santé, les compétences de vie, l'éducation financière et les normes de genre. 


\section{AGENDA DE LA FF : FACILITER LES GROUPES ET L'ENGAGEMENT COMMUNAUTAIRE (suite)}

Jour 1

\begin{tabular}{|c|c|c|c|}
\hline Horaires & Session & Objectif & Facilitateur \\
\hline $8.30-9.00$ & $\begin{array}{l}\text { Inscription, mise en train/ } \\
\text { introduction/programme } \\
\text { de formation et } \\
\text { logistique de formation }\end{array}$ & $\begin{array}{l}\text { - } \quad \text { Recueillir les coordonnées des } \\
\text { participantes } \\
\text { - } \quad \text { Partager la logistique de la formation }\end{array}$ & \\
\hline $9.00-10.00$ & $\begin{array}{l}\text { Objectifs de formation/ } \\
\text { règles de base } \\
\text { Attentes des participantes }\end{array}$ & $\begin{array}{l}\text { - Lire avec les participantes les objectifs } \\
\text { et le programme de formation }\end{array}$ & \\
\hline $10.00-10.30$ & \multicolumn{2}{|r|}{ PAUSE CAFÉ } & \\
\hline $10.30-11.30$ & $\begin{array}{l}\text { Bref historique sur } \\
\text { l'organisation de mise en } \\
\text { œuvre, le programme, la } \\
\text { conception de la } \\
\text { recherche et les } \\
\text { objectifs, y compris les } \\
\text { éléments d'interventions }\end{array}$ & $\begin{array}{l}\text { - Comprendre le travail de l'organisation de mise } \\
\text { en œuvre } \\
\text { Comprendre l'objectif, la théorie du } \\
\text { changement, la recherche et les composantes } \\
\text { du programme }\end{array}$ & \\
\hline $11.30-12: 30$ & Le mentorat et ses rôles & $\begin{array}{l}\text { - Comprendre ce qu'est le mentorat, pourquoi } \\
\text { devenir mentore, les rôles d'une mentore, les } \\
\text { qualités et les responsabilités, les rôles spécifiques } \\
\text { des mentores dans le programme }\end{array}$ & \\
\hline $12.30-13.00$ & $\begin{array}{l}\text { Présenter le concept de } \\
\text { brise-glace et les } \\
\text { activités énergisantes }\end{array}$ & $\begin{array}{l}\text { - Comprendre le rôle de l'utilisation des activités } \\
\text { brise-glace/énergisantes en groupe } \\
\text { - } \quad \text { Pratiquer différents types d'activités }\end{array}$ & \\
\hline $13.00-14.00$ & \multicolumn{2}{|r|}{ PAUSE DÉJEUNER } & \\
\hline $14.00-15.00$ & $\begin{array}{l}\text { Comprendre le concept } \\
\text { d'engagement total } \\
\text { avec les participantes }\end{array}$ & $\begin{array}{l}\text { Pratiquer le langage de l'engagement, développer une } \\
\text { liste de techniques pour promouvoir un cadre } \\
\text { d'apprentissage sûr, faire des éloges; préférer les } \\
\text { questions ouvertes par rapport aux questions fermées }\end{array}$ & \\
\hline $15.00-16.00$ & $\begin{array}{l}\text { Développer l'esprit } \\
\text { d'équipe/la confiance }\end{array}$ & $\begin{array}{l}\text { Instaurer la confiance et promouvoir le travail } \\
\text { d'équipe; pratiquer des exercices de team-building }\end{array}$ & \\
\hline $16.00-16.30$ & Faire une synthèse & - Les participantes évaluent la session de la journée & \\
\hline
\end{tabular}

Jour 2

\begin{tabular}{|c|c|c|c|}
\hline Horaires & Session & Objectif & Facilitateur \\
\hline $8.00-8.30$ & Récapitulatif de la veille & $\begin{array}{l}\text { - Les participantes actualise le contenu de la veille et } \\
\text { révise l'agenda du jour } 2\end{array}$ & \\
\hline $8.30-9.30$ & Lire votre audience & $\begin{array}{l}\text { - Mettre en évidence les besoins des différents } \\
\text { segments clés des filles et comment surmonter les } \\
\text { défis de leur implication totale }\end{array}$ & \\
\hline $9.30-10.30$ & $\begin{array}{l}\text { Qui impliquer dans le } \\
\text { travail avec les filles/ } \\
\text { comment se mobiliser } \\
\text { pour leur soutien }\end{array}$ & $\begin{array}{l}\text { - Identifier différents segments de filles, développer } \\
\text { une liste des principales parties prenantes dans le } \\
\text { travail avec différents segments de filles, identifier les } \\
\text { défis liés au travail et comment les surmonter }\end{array}$ & \\
\hline $10.30-10.45$ & & PAUSE CAFÉ & \\
\hline $10.45-12.00$ & Clarification de valeurs & $\begin{array}{l}\text { - Explorer et clarifier les croyances et attitudes des } \\
\text { participantes à l'égard des problèmes de santé } \\
\text { reproductive chez les adolescentes } \\
\text { Utilisez le modèle « d'accord », «pas d'accord », «pas } \\
\text { sûr » }\end{array}$ & \\
\hline
\end{tabular}




\begin{tabular}{|c|c|c|}
\hline $12.00-13.00$ & $\begin{array}{l}\text { Renforcement des } \\
\text { compétences de } \\
\text { facilitation pour le } \\
\text { changement souhaité }\end{array}$ & $\begin{array}{l}\text { Comprendre le rôle de la facilitation } \\
\text { Techniques de facilitation } \\
\text { Qualités d'un bon facilitateur } \\
\text { - Travail de groupe : Identifier les besoins } \\
\text { uniques des adolescentes vulnérables } \\
\text { Mettre en évidence les compétences de } \\
\text { facilitation les plus appropriées aux besoins } \\
\text { uniques des adolescentes }\end{array}$ \\
\hline $13.00-14.00$ & & PAUSE DÉJEUNER \\
\hline 14.00-15.00 & Dynamique de groupe & $\begin{array}{l}\text { - Comprendre les défis et comment gérer les groupes, } \\
\text { animer les groupes, maintenir les filles dans un } \\
\text { groupe, résolution des conflits en travaillant avec les } \\
\text { filles/tuteurs et la communauté dans son ensemble }\end{array}$ \\
\hline 15.00-16.00 & $\begin{array}{l}\text { Planification de la } \\
\text { sécurité }\end{array}$ & $\begin{array}{l}\text { - Comprendre ce qu'est un espace sûr, comment } \\
\text { identifier les espaces sûrs et concevoir des itinéraires } \\
\text { sûrs pour s'y rendre } \\
\text { Travail de groupe : Réflexion sur d'éventuels espaces } \\
\text { sûrs dans la communauté }\end{array}$ \\
\hline $16.00-16.30$ & $\begin{array}{l}\text { Distribution des pro- } \\
\text { grammes pour examen }\end{array}$ & \\
\hline $16.30-17.00$ & & SYNTHĖSE ET CLÔTURE \\
\hline
\end{tabular}

Jour 3

\begin{tabular}{|c|c|c|c|}
\hline Horaires & Session & Objectif & Facilitateur \\
\hline $8.00-8.30$ & Récapitulatif de la veille & $\begin{array}{l}\text { - Les participantes actualise le contenu de la veille et } \\
\text { révise l'agenda du jour } 3\end{array}$ & \\
\hline $8.30-9.30$ & $\begin{array}{l}\text { Mettre en place un } \\
\text { système de référence }\end{array}$ & $\begin{array}{l}\text { - Comprendre ce qu'est la référence et les services } \\
\text { qui sont nécessaires pour et parcourir la liste } \\
\text { des services disponibles dans la communauté }\end{array}$ & \\
\hline $9.30-10.30$ & $\begin{array}{l}\text { Aperçu des programmes } \\
\text { de formation sur la santé } \\
\text { et les compétences de vie }\end{array}$ & $\begin{array}{l}\text { - Revoir le programme de santé, y compris la } \\
\text { structure, les thèmes et le déroulement des sessions } \\
\text { - } \quad \text { Identifier les sujets difficiles }\end{array}$ & \\
\hline $10.30-11.00$ & \multicolumn{2}{|r|}{ PAUSE CAFÉ } & \\
\hline $11.00-12.00$ & $\begin{array}{l}\text { Aperçu du programme } \\
\text { sur l'éducation financière }\end{array}$ & $\begin{array}{l}\text { - Examiner le programme d'éducation financière, y } \\
\text { compris la structure, les thèmes et le déroulement } \\
\text { des sessions } \\
\text { - } \quad \text { Identifier les thèmes difficiles }\end{array}$ & \\
\hline $12.00-13.00$ & $\begin{array}{l}\text { Aperçu du programme } \\
\text { de formation sur les } \\
\text { normes de genre }\end{array}$ & $\begin{array}{ll}- & \text { Revoir le programme sur les normes de genre, y compris } \\
\text { la structure, les thèmes et le déroulement des } \\
\text { sessions } \\
\text { - } \quad \text { ldentifier les thèmes difficiles }\end{array}$ & \\
\hline $13.00-14.00$ & \multicolumn{2}{|r|}{ PAUSE DÉJEUNER } & \\
\hline $14.00-15.00$ & Être assertive & & \\
\hline $15.00-16.00$ & $\begin{array}{l}\text { Gérer le stress, la } \\
\text { colère et les conflits }\end{array}$ & & \\
\hline $16.00-16.30$ & \multicolumn{2}{|r|}{ SYNTHĖSE } & \\
\hline
\end{tabular}




\section{AGENDA DE LA FF : FACILITER LES GROUPES ET L'ENGAGEMENT COMMUNAUTAIRE (suite)}

Jour 4

\begin{tabular}{|c|c|c|c|}
\hline Horaires & Session & Objectif & Facilitateur \\
\hline $8.00-8.30$ & Récapitulatif de la veille & $\begin{array}{l}\text { Les participantes actualise le contenu de la } \\
\text { veille et révise l'agenda du jour } 4\end{array}$ & \\
\hline $8.30-9.30$ & $\begin{array}{l}\text { Connaître la différence } \\
\text { entre les besoins et les } \\
\text { désirs }\end{array}$ & & \\
\hline $10.30-11.00$ & & PAUSE CAFÉ & \\
\hline $11.00-12.00$ & $\begin{array}{l}\text { Explorer les options pour } \\
\text { gagner de l'argent }\end{array}$ & & \\
\hline $12.00-13.00$ & Adolescence et puberté & & \\
\hline 13.00-14.00 & & PAUSE DÉJEUNER & \\
\hline 14.00-15.00 & Sexualité et comportement & & \\
\hline $15.00-16.00$ & $\begin{array}{l}\text { Les jeunes et les } \\
\text { contraceptifs }\end{array}$ & & \\
\hline $16.00-16.30$ & \multicolumn{2}{|c|}{ SYNTHĖSE ET CLÔTURE } & \\
\hline
\end{tabular}

Jour 5

\begin{tabular}{|c|c|c|c|}
\hline Horaires & Session & Objectif & Facilitateur \\
\hline $8.00-8.30$ & Récapitulatif de la veille & $\begin{array}{l}\text { Les participantes actualise le contenu de la } \\
\text { veille et révise l'agenda du jour } 5\end{array}$ & \\
\hline $8.30-9.30$ & Les grossesses précoces & & \\
\hline $9.30-10.30$ & Drogues et toxicomanie & & \\
\hline 10.30-11.00 & & PAUSE CAFÉ & \\
\hline $11.00-12.00$ & $\begin{array}{l}\text { Devenir un champion } \\
\text { du genre }\end{array}$ & & \\
\hline $12.00-13.00$ & Apprendre sur les banques & & \\
\hline 13.00-14.00 & & PAUSE DÉJEUNER & \\
\hline 14.00-15.00 & Les comptes bancaires & & \\
\hline $15.00-16.00$ & $\begin{array}{l}\text { Faire face aux revers de } \\
\text { l'épargne }\end{array}$ & & \\
\hline $16.00-16.30$ & \multicolumn{2}{|c|}{ SYNTHĖSE ET CLÔTURE } & \\
\hline
\end{tabular}

Jour 6

\begin{tabular}{|l|l|l|l|}
\hline Horaires & Session & Objectif & Facilitateur \\
\hline $8.00-8.30$ & Récapitulatif de la veille & $\begin{array}{l}\text { Les participantes actualise le contenu de la } \\
\text { veille et révise l'agenda du jour 6 }\end{array}$ & \\
\hline $8.30-9.30$ & Comprendre le genre & & \\
\hline $9.30-10.30$ & $\begin{array}{l}\text { Comprendre le pouvoir et } \\
\text { le statut }\end{array}$ & & \\
\hline $10.30-11.00$ & \multicolumn{1}{|c|}{ PAUSE CAFÉ } & \\
\hline $11.00-12.00$ & $\begin{array}{l}\text { Comprendre la violence } \\
\text { contre femmes et filles }\end{array}$ & & \\
\hline $12.00-13.00$ & La communauté idéale & & \\
\hline
\end{tabular}




\begin{tabular}{|l|l|l|l|}
\hline $13.00-14.00$ & & \\
\hline $14.00-15.00$ & $\begin{array}{l}\text { Revue du thème } \\
\text { difficile identifié }\end{array}$ & $\begin{array}{l}\text { PAUSE DÉJEUNER } \\
\text { constructif }\end{array}$ & \\
\hline $15.00-16.00$ & \multicolumn{2}{|c|}{ SYNTHĖSE ET CLÔTURE } \\
\hline $16.00-16.30$ & $\begin{array}{l}\text { Acquérir des compétences pratiques pour } \\
\text { fournir de feedback constructifs }\end{array}$ & \\
\hline
\end{tabular}

Jour 7

\begin{tabular}{|c|c|c|c|}
\hline Horaires & Session & Objectif & Facilitateur \\
\hline $8.00-8.30$ & Récapitulatif de la veille & $\begin{array}{l}\text { - Les participantes actualise le contenu } \\
\text { de la veille et révise l'agenda du jour } 7\end{array}$ & \\
\hline $8.30-10.30$ & $\begin{array}{l}\text { Attribution de } \\
\text { sessions pratiques }\end{array}$ & $\begin{array}{l}\text { Devoir - parcourir les programmes de } \\
\text { formations sur la santé et les compé- } \\
\text { tences de vie, l'éducation financière et les } \\
\text { normes de genre et noter les questions } \\
\text { Attribuer aux participantes des séances } \\
\text { pratiques - évaluer leurs compétences de } \\
\text { facilitation. } \\
\text { Diviser les participantes en } 7 \text { équipes de } 3\end{array}$ & \\
\hline $10.30-11.00$ & & PAUSE CAFÉ & \\
\hline $11.00-12.30$ & $\begin{array}{l}\text { Session pratique } \\
\text { Les participantes aux } \\
\text { séances pratiques } \\
\text { présentent leurs sujets : } \\
\text { Équipe } 1: \text { [Titre de la session] } \\
\text { Feedback des groupes } \\
\text { sur les sessions pratiques }\end{array}$ & $\begin{array}{l}\text { - Les participantes mettent en pratique } \\
\text { leurs compétences de facilitation et se } \\
\text { familiarisent avec le curriculum } \\
\text { Les participantes identifient les } \\
\text { compétences de facilitation utilisées pour } \\
\text { faciliter efficacement } \\
\text { - Les participantes discutent des défis } \\
\text { rencontrés pendant la facilitation }\end{array}$ & \\
\hline $12.30-13.30$ & & PAUSE DÉJEUNER & \\
\hline $13.30-14.30$ & $\begin{array}{l}\text { Session pratique } \\
\text { Les participantes } \\
\text { présentent leurs sujets. } \\
\text { Équipe } 2: \text { [Titre de la session] } \\
\text { Feedback des groupes } \\
\text { sur les sessions pratiques }\end{array}$ & $\begin{array}{l}\text { - Les participantes mettent en pratique } \\
\text { leurs compétences de facilitation et se } \\
\text { familiarisent avec le curriculum } \\
\text { Les participantes identifient les } \\
\text { compétences de facilitation utilisées } \\
\text { pour faciliter efficacement } \\
\text { - Les participantes discutent des défis } \\
\text { rencontrés pendant la facilitation }\end{array}$ & \\
\hline $14.30-16.00$ & $\begin{array}{l}\text { Session pratique } \\
\text { Les participantes aux } \\
\text { séances pratiques } \\
\text { présentent leurs sujets: } \\
\text { Équipe } 3: \text { [itre de la session] } \\
\text { Feedback des groupes } \\
\text { sur les sessions pratiques }\end{array}$ & $\begin{array}{l}\text { - Les participantes mettent en pratique } \\
\text { leurs compétences de facilitation et se } \\
\text { familiarisent avec le curriculum } \\
\text { Les participantes identifient les } \\
\text { compétences de facilitation utilisées } \\
\text { pour faciliter efficacement } \\
\text { - Les participantes discutent des défis } \\
\text { rencontrés pendant la facilitation }\end{array}$ & \\
\hline $16.00-16.30$ & \multicolumn{2}{|c|}{ SYNTHĖSE ET CLÔTURE } & \\
\hline
\end{tabular}




\section{AGENDA DE LA FF : FACILITER LES GROUPES ET L'ENGAGEMENT COMMUNAUTAIRE (suite)}

Jour 8

\begin{tabular}{|c|c|c|c|}
\hline Horaires & Session & Objectifs & Facilitateur \\
\hline $8.00-8.30$ & Récapitulatif de la veille & $\begin{array}{l}\text { - Les participantes actualise le contenu de la } \\
\text { veille et révise l'agenda du jour } 8\end{array}$ & \\
\hline $8.30-10.30$ & $\begin{array}{l}\text { Session pratique } \\
\text { Les participantes aux } \\
\text { séances pratiques } \\
\text { présentent leurs sujets: } \\
\text { Équipe } 4: \text { [Titre de la session] } \\
\text { Feedback des groupes } \\
\text { sur les sessions pratiques }\end{array}$ & $\begin{array}{l}\text { - Les participantes mettent en pratique } \\
\text { leurs compétences de facilitation et se } \\
\text { familiarisent avec le curriculum } \\
\text { Les participantes identifient les } \\
\text { compétences de facilitation utilisées pour } \\
\text { faciliter efficacement } \\
\text { - Les participantes discutent des défis } \\
\text { rencontrés pendant la facilitation }\end{array}$ & \\
\hline $10.30-11.00$ & & PAUSE CAFÉ & \\
\hline $11.00-12.30$ & $\begin{array}{l}\text { Session pratique } \\
\text { Les participantes aux } \\
\text { séances pratiques } \\
\text { présentent leurs sujets : } \\
\text { Équipe } 5: \underline{\text { [Titre de la session] }} \\
\text { Feedback des groupes sur } \\
\text { les sessions pratiques }\end{array}$ & $\begin{array}{l}\text { - Les participantes mettent en pratique } \\
\text { leurs compétences de facilitation et se } \\
\text { familiarisent avec le curriculum } \\
\text { - Les participantes identifient les } \\
\text { compétences de facilitation utilisées pour } \\
\text { faciliter efficacement } \\
\text { Les participantes discutent des défis } \\
\text { rencontrés pendant la facilitation }\end{array}$ & \\
\hline $12.30-13.30$ & & PAUSE DÉJEUNER & \\
\hline $13.30-14.30$ & $\begin{array}{l}\text { Session pratique } \\
\text { Les participantes aux } \\
\text { séances pratiques } \\
\text { présentent leurs sujets: } \\
\text { Équipe } 6 \text { :[Titre de la session] } \\
\text { Feedback des groupes } \\
\text { sur les sessions pratiques }\end{array}$ & $\begin{array}{l}\text { - Les participantes mettent en pratique } \\
\text { leurs compétences de facilitation et se } \\
\text { familiarisent avec le curriculum } \\
\text { - Les participantes identifient les } \\
\text { compétences de facilitation utilisées pour } \\
\text { faciliter efficacement } \\
\text { - Les participantes discutent des défis } \\
\text { rencontrés pendant la facilitation }\end{array}$ & \\
\hline $14.30-16.00$ & $\begin{array}{l}\text { Session pratique } \\
\text { Les participantes aux } \\
\text { séances pratiques } \\
\text { présentent leurs sujets: } \\
\text { Équipe } 7: \text { [Titre de la session] } \\
\text { Feedback des groupes } \\
\text { sur les sessions pratiques }\end{array}$ & $\begin{array}{l}\text { - Les participantes mettent en pratique } \\
\text { leurs compétences de facilitation et se } \\
\text { familiarisent avec le curriculum } \\
\text { - Les participantes identifient les } \\
\text { compétences de facilitation utilisées pour } \\
\text { faciliter efficacement } \\
\text {-es participantes discutent des défis } \\
\text { rencontrés pendant la facilitation }\end{array}$ & \\
\hline $16.00-16.30$ & \multicolumn{2}{|c|}{ SYNTHĖSE ET CLÔTURE } & \\
\hline
\end{tabular}

Jour 9

\begin{tabular}{|l|l|l|l|}
\hline Horaires & Session & Objectif & Facilitateur \\
\hline $8.00-8.15$ & $\begin{array}{l}\text { Activité énergisante/ } \\
\text { «Ménage » }\end{array}$ & $\begin{array}{l}\text { - Offrir aux participantes l'expérience des } \\
\text { énergisants du manuel Energizer/d'autres } \\
\text { exercices énergisants suggérés }\end{array}$ & \\
\hline $8.15-8.30$ & Feedback sur le jour 8 & Faire une évaluation continue de l'atelier & \\
\hline $8.30-10.30$ & $\begin{array}{l}\text { Introduction à la } \\
\text { cartographie de la } \\
\text { sécurité }\end{array}$ & $\begin{array}{l}\text { Comprendre l'importance de la cartographie } \\
\text { de la sécurité pour les groupes de filles } \\
\text { Apprendre la cartographie de la sécurité }\end{array}$ & \\
\hline $10.30-11.00$ & \multicolumn{1}{|c|}{ PAUSE CAFÉ } & \\
\hline
\end{tabular}




\begin{tabular}{|c|c|c|}
\hline $11.00-13.00$ & $\begin{array}{l}\text { Rôles de la mentore du } \\
\text { programme : envoi des } \\
\text { invitations, rencontre } \\
\text { d'introduction, rencontres } \\
\text { dans un espace sûr et } \\
\text { recrutement et rétention } \\
\text { des filles }\end{array}$ & $\begin{array}{l}\text { - } \quad \text { Récapitulatif sur le rôle des mentores } \\
\text { Expliquer le processus d'envoi des } \\
\text { invitations et le rôle des mentores } \\
\text { - Introduire le protocole de recrutement et } \\
\text { de rétention } \\
\text { - } \quad \text { Présenter le modèle de pont }\end{array}$ \\
\hline $13.00-14.00$ & \multicolumn{2}{|r|}{ PAUSE DÉJEUNER } \\
\hline $14.00-15.00$ & Contrôle et évaluation & $\begin{array}{ll}\text { - } & \text { Introduire des outils de suivi et } \\
& \text { d'évaluation } \\
\text { - } & \text { Registres/présence } \\
\text { - } & \text { Rapports }\end{array}$ \\
\hline $15.00-16.30$ & $\begin{array}{l}\text { Évaluation et prochaines } \\
\text { étapes }\end{array}$ & $\begin{array}{l}\text { - } \quad \text { Résumer les réalisations de l'atelier } \\
\text { Expliquer ce que l'on attend des } \\
\text { mentores à l'avenir }\end{array}$ \\
\hline \multirow[t]{2}{*}{$16.30-17.00$} & Questionnaire post-test & $\begin{array}{l}\text { Mesurer les niveaux de connaissances } \\
\text { acquises par les participantes à la } \\
\text { formation }\end{array}$ \\
\hline & \multicolumn{2}{|r|}{ CÉRÉMONIE DE CLÔTURE } \\
\hline
\end{tabular}

\section{JOUR 10}

\begin{tabular}{|c|c|c|c|}
\hline Horaires & Session & Objectif & Facilitateur \\
\hline $8.00-8.30$ & Récapitulatif de la veille & $\begin{array}{l}\text { Les participantes actualise le contenu de la veille } \\
\text { et révise l'agenda du jour } 10\end{array}$ & \\
\hline $8.30-10.30$ & $\begin{array}{l}\text { La collecte de données sur } \\
\text { les téléphones portables }\end{array}$ & & \\
\hline $10.30-11.00$ & \multicolumn{3}{|c|}{ PAUSE CAFÉ } \\
\hline $11.00-13.00$ & $\begin{array}{l}\text { Pratique de collecte de } \\
\text { données sur les portables }\end{array}$ & & \\
\hline $13.00-14.00$ & \multicolumn{3}{|c|}{ PAUSE DÉJEUNER } \\
\hline $14.00-15.00$ & $\begin{array}{l}\text { Recrutement/rencontres } \\
\text { d'introduction }\end{array}$ & & \\
\hline $15.00-16.00$ & $\begin{array}{l}\text { Évaluation des participantes/ } \\
\text { prochaines étapes et clôture }\end{array}$ & & \\
\hline
\end{tabular}




\section{FORMULAIRE DES ÉVALUATIONS QUOTIDIENNES}

Il suffit de quelques questions pour savoir à quel point la formation est efficace. Ces cinq questions ont été posées aux mentores à la fin de chaque journée d'une séance de formation de plusieurs jours pour évaluer ce que les mentores ont appris et apprécié de la formation. Faire réfléchir les participantes à la fin de chaque journée permet aux animatrices d'apporter des changements pour les jours suivants et de mettre davantage l'accent sur les domaines qui nécessitent plus d'explications. Ces questions peuvent être imprimées et distribuées, ou chaque participante peut répondre sur un post-it ou une fiche, avec des réponses numérotées.

Date:

1. Qu'avez-vous apprécié le plus aujourd'hui?

2. Qu'avez-vous appris au cours des sessions d'aujourd'hui que vous prévoyez utiliser dans votre travail ?

3. Y a-t-il quelque chose que vous n'avez pas compris pendant les séances d'aujourd'hui ? Veuillez donner des exemples spécifiques.

4. Quelle est la chose la plus importante que vous avez apprise aujourd'hui (en termes de connaissances ou compétences)?

5. Y a-t-il autre chose que vous aimeriez ajouter? 


\section{PROGRAMME DES RENCONTRES MENSUELS DES MENTORES}

Après une formation initiale, il est généralement utile de réunir les mentores régulièrement, par exemple tous les mois, pour discuter entre elles (et avec un superviseur) de ce qui se passe dans leur groupe et des problèmes pour lesquels elles pourraient avoir besoin d'aide. Les rencontres mensuels sont un moment important pour les mentores pour développer leurs compétences et apprendre les unes des autres. Voici un exemple d'agenda des rencontres mensuels pour l'appui de la mentore. II comprend une mise à jour sur les rencontres dans un espace sûr du mois précédent, les succès, les défis, les solutions suggérées, les expériences de visites à domicile, la revue des sessions du curriculum du mois précédent, une collection d'histoires de réussite, un aperçu de l'outil de participation, une formation pour les sessions à venir, et un examen de la soumission des rapports mensuels.

\begin{tabular}{|c|c|c|}
\hline Durée & Ordre du jour & Facilitateur \\
\hline $9.05-9.10$ & Bienvenu/Introduction & \\
\hline $9.10-11.00$ & $\begin{array}{l}\text { Mises à jour sur les rencontres sur les espaces sûrs du mois précédent, y } \\
\text { compris les succès, les défis, les solutions suggérées et les expériences } \\
\text { des visites à domicile effectuées. Enregistrez-le pour chaque session dans } \\
\text { le rapport mensuel du coordinateur de site afin que nous puissions } \\
\text { recueillir du feedback de toutes les sessions sur tous les sites. }\end{array}$ & \\
\hline $11.00-11.45$ & $\begin{array}{l}\text { Revue des sessions du curriculum animées le mois précédent en } \\
\text { examinant les domaines suivants : } \\
\text { - Orientations peu claires dans le programme } \\
\text { - Niveau d'intérêt/engagement des filles/parents } \\
\text { - Compréhension globale du sujet à la fin de la session } \\
\text { - Temps alloué aux exercices } \\
\text { - } \quad \text { Défis/adaptations suggérés }\end{array}$ & \\
\hline $11.45-12.10$ & $\begin{array}{l}\text { Histoires de réussite des mentores } \\
\text { - } \quad \text { Les défis rencontrés } \\
\text { - } \quad \text { Critères } \\
\text { - } \quad \text { Suivi possible }\end{array}$ & \\
\hline $12.10-12.30$ & $\begin{array}{l}\text { Examen de l'outil de contrôle de présence } \\
\text { - Rencontres manqués } \\
\text { - Nouveaux ajouts } \\
\text { - Vérification du formulaire (si vous utilisez la collecte électronique de } \\
\text { données) }\end{array}$ & \\
\hline $12.30-13.00$ & PAUSE & \\
\hline
\end{tabular}




\section{PROGRAMME DES RENCONTRES MENSUELS} DES MENTORES (suite)

\begin{tabular}{|l|l|l|}
\hline $13: 00-13: 40$ & Formation sur le curriculum : session à venir & \\
\hline $13: 40-14: 10$ & Planification des rencontres d'engagement communautaire & \\
\hline $14: 10-14: 50$ & Formation sur le curriculum : session à venir & $\begin{array}{l}\text { Planification de la remise des diplômes dans un espace sûr : durée, } \\
\text { structure et invités (à l'approche de la fin du cycle du programme) }\end{array}$ \\
\hline $14: 50-15: 20$ & Soumission des rapports mensuels & \\
\hline $15: 20-15: 30$ & Questions administratives & \\
\hline $15: 40-16: 00$ & Divers & \\
\hline
\end{tabular}




\section{Cartes «Neuf Actes »}

Ceci décrit une activité qui peut être réalisée dans le cadre de la formation des mentores décrivant neuf principes clés d'une relation de mentorat réussie. Les cartes doivent être imprimées suffisamment grandes pour être visibles comme rappel sur le mur pendant la formation

\section{\# 1 : SOYEZ PROACTIVE}

N'attendez pas que les filles vous contactent.

\section{\# 4 : SOYEZ CONFIANT}

Chacune de vous a quelque chose d'important à offrir à l'autre.

\section{\# 7 : SOYEZ RÉACTIVE}

Agissez selon ce que vous avez prévu.

\section{\# 2 : NÉGOCIEZ UN ENGAGEMENT}

Acceptez d'avoir des contacts réguliers à des heures fixes.

\section{\# 5 : COMMUNIQUEZ}

Partagez ouvertement vos connaissances et votre expérience.

\section{\# 8 : SOYEZ ACCESSIBLE}

Ayez une politique de porte ouverte ou de téléphone ouvert.

\section{\# 3 : ÉTABLISSEZ UN} RAPPORT

Apprenez le plus possible les unes sur les autres.

\section{\# 6 : SOYEZ UN BON ÉCOUTEUSE}

Écoutez ce que les filles vous disent.

\section{\# 9 : PRENEZ LA RESPONSABILITÉ}

Il en faut deux pour avoir une relation de mentorat réussie. 


\section{COMPÉTENCES EN FACILITATION}

Cette activité de 60 minutes permet aux participantes d'apprendre et de mettre en pratique une variété de compétences d'animation qu'elles utiliseront en tant que mentores dans le programme.

Durée : 60 minutes

Matériaux : Flip chart, marqueur, chaises, balle de "koosh », poule en caoutchouc, balle extensible, documents

\section{Facilitateur :}

\section{Objectifs :}

- Les participantes apprennent différents types de compétences de facilitation

- Les participantes comprennent comment appliquer ces compétences lors des sessions sur les espaces sûrs

Processus : Le facilitateur doit préparer cette session "à l'avance » en demandant qu'au moins 4 volontaires se constituent en public pendant la session. Le facilitateur présente les 14 « être » (critères) d'une facilitation réussie en faisant un jeu de rôle de chaque "être » et demander aux participantes de deviner la compétence, et de discuter ou expliquer.

\section{Les 14 "être " d’une facilitation réussie}

1. Être passionnée! Les participantes seront deux fois plus émotives que vous. Si vous êtes enthousiaste, les participantes seront deux fois plus enthousiastes. Si vous êtes ennuyeux, les participantes s'ennuieront deux fois plus. À ÉVITER : l'animatrice semble s'ennuyer pendant qu'elle anime une séance, par exemple, l'expression de son visage est sombre, soit elle bâille et parle à voix basse.

2. Être un éléphant ! Un éléphant a de grandes oreilles et une petite bouche : écoutez ce que disent les participantes, laissez-les parler. Un bon facilitateur parle 10 pour cent du temps. Posez des questions, renvoyez les questions des participantes aux participantes.

À ÉVITER : l'animatrice parle tout au long de la session sans donner la possibilité à aucune participante de dire quelque chose.
3. Être neutre ! N'imposez pas vos valeurs aux participantes. Le programme permet aux participantes de partager librement leurs valeurs sans l'influence des valeurs de la mentore. À ÉVITER : l'animatrice impose ses valeurs aux participantes, par exemple, en leur disant que les relations sexuelles avant le mariage sont un péché et qu'elles devraient toutes s'abstenir de toute activité sexuelle.

4. Être prête ! Préparez-vous en expliquant les activités aux autres. Rassemblez le matériel dont vous aurez besoin (soyez créative si vous n'avez pas le matériel) et trouvez les réponses aux questions auxquelles vous n'avez pas pu répondre lors de la session précédente.

À ÉVITER : l'animatrice ne semble pas prête pendant la facilitation.

5. Être en contact visuel ! Établissez un contact visuel avec toutes les participantes signifie qu'elles sont à l'écoute. Si vous êtes à l'extérieur, assurez-vous que le soleil est dans le dos des participantes et dans vos yeux.

A ÉVITER : l'animatrice regarde vers le bas, le haut ou sur le côté pendant la facilitation et s'agite beaucoup.

6. Être honnête! Répondez honnêtement à toutes les questions. Ne vous sentez pas mal si vous ne connaissez pas la réponse à une question. Dites simplement que vous ne savez pas, mais vous ferez tout votre possible pour le savoir. À ÉVITER : une participante pose une question à la mentore et elle répond par de fausses informations, par exemple, peut-on contracter le $\mathrm{VIH}$ en partageant des assiettes, des tasses ou une poignée de main? Hmmm ! Je ne suis pas vraiment sûre, mais oui, on peut être infecté. 
PLANIFICATEUR

\section{7. Être au même niveau que les participantes !}

- Discussions sensibles : si les participantes rient du mot « vagin », laissez-les rire. Si elles sont autorisées à rire, elles seront plus enclines à en parler.

- Langue : n'utilisez pas de grands mots qu'elles ne comprendront pas. Traduisez si nécessaire pour que toutes les participantes comprennent de quoi vous parlez.

- Soyez consciente de votre audience et mettezvous à sa place (c'est-à-dire en travaillant avec un groupe de filles vulnérables).

Á ÉVITER : pendant l'animation, le facilitateur utilise de grands mots comme l'anatomie corporelle, l'inflation ou le surplus.

\section{8. Être cohérente !}

Il est facile d'enseigner aux meilleures participantes. Assurez-vous d'obtenir la participation de toutes. Posez des questions faciles ou simples aux participantes calmes pour impliquer tout le monde.

À ÉVITER : la mentore interroge la même participante pour obtenir des réponses parce qu'elle est la plus active et ignore complètement celles qui ne participent pas.

9. Être sensible !

Sachez gérer les questions sensibles, en particulier lors des discussions quand les participantes s'ouvrent et partagent leur expérience personnelle.

À ÉVITER : l'animatrice présente un jeu qui permet aux participantes d'être à l'aise pour discuter ou partager des questions sensibles.

\section{0. Être positive !}

Restez positive maintient les niveaux d'énergie et crée un bon environnement pour la critique constructive et l'apprentissage. Donnez une critique constructive aux participantes entre deux éloges/points positifs.

À ÉVITER : le facilitateur doit féliciter les participantes qui répondent correctement et également remercier celles qui participent.

\section{1. Être entendue !}

Le volume, le volume, et le volume! Assurez-vous que votre voix est entendue par toutes les participantes.

À ÉVITER : l'animatrice parle à voix basse.

\section{2. Être ponctuelle !}

Le programme prévoit du temps pour chaque activité, donc si vous faites une activité de 2 heures qui devrait être faite en 20 minutes, vos participantes s'ennuieront et vous manquerez d'effectuer d'autres activités importantes.

\section{3. Être au contrôle!}

- Les participantes auront tellement de plaisir qu'elles oublieront qu'elles apprennent. Les courtes activités de réveil aident à contrôler les participantes afin que chacune prête attention à ce que vous dites.

- Souvenez-vous des autres "être " (en particulier le $1,5,7,8,10,11$ et 12) pour être au contrôle efficacement.

À ÉVITER : l'animatrice doit présenter une courte activité de réveil.

\section{4. Être concentrée!}

Ne laissez pas les commentaires hors sujet et les questions des participantes vous empêcher de livrer les messages clés de l'activité.

À ÉVITER : une participante pose une question qui ne fait pas partie du sujet de discussion et essaie de distraire le groupe en se concentrant un peu sur un sujet différent. Le facilitateur remet la discussion sur la bonne voie en répondant à la question ou en disant au groupe que ce sera discuté en détail dans une autre session. 


\section{MÉTHODOLOGIES DE FORMATION}

\section{PARTIE I : FACILITATION DES ESPACES SÛRS}

\section{Objectif}

Renforcer la capacité des mentores à faciliter la mise en œuvre d'espaces sûrs et à offrir efficacement un curriculum aux filles du programme

Durée : 30 minutes au total

Matériel : Flip chart, marqueur, documents, projecteur

Processus : L'animatrice explique les objectifs de l'atelier aux participantes à l'aide de PowerPoint et écrit également les objectifs sur un flip chart à l'avance afin de les coller au mur.

Durée : 15 minutes

\section{Objectifs spécifiques}

- Former les mentores à comprendre les concepts du programme.

- Fournir aux mentores des informations complètes sur les compétences de vie, la santé et droits sexuels et reproductifs et la gestion financière.

- Former les mentores pour faciliter une gamme de méthodologies interactives à utiliser pendant les sessions sur l'espace sûr.

- Outiller les mentores des connaissances et des compétences nécessaires pour fournir des conseils et un soutien social aux AJF participantes aux séances sur l'espace sûr.

Processus: Le formateur explique très brièvement la formation expérientielle et hautement interactive que les participantes utiliseront lors de leurs sessions hebdomadaires avec les filles comme prévu dans le programme. C'est le bon moment pour évoquer le vieil adage : «Dites-moi... j'oublie, montrez-moi... je me souviens, impliquez-moi... je comprends. » Expliquez que cette formation sera une formation d'engagement, d'apprentissage par l'expérience.
Les méthodes d'enseignement participatif pour renforcer les compétences et influencer les attitudes sont les suivantes:

- Discussions de groupe

- Réflexion

- Démonstration et pratique guidée

- Jeu de rôle

- Petits groupes

- Jeux éducatifs et simulations

- Études de cas

- Narration

- Débats

L'animatrice divise ensuite le groupe en quatre petits groupes en leur demandant de compter 1, 2, 3, 4 et en les regroupant en fonction de leur nombre. On attribue des tâches aux groupes et leur donne 15 minutes pour préparer.

\section{Groupe 1 : Jeu de rôle}

Instructions

- Décidez en tant que groupe, d'une situation à jouer.

- Sélectionnez des acteurs.

- Donnez des instructions aux acteurs.

- Commencez le jeu de rôle.

- Discutez de ce qui s'est passé.

\section{Groupe 2 : Jeux/simulations}

Instructions

- En équipe, jouez un petit match.

- Cela doit être agréable et peu importe qui gagne.

- Discutez brièvement immédiatement après le match.

\section{Groupe 3 : Débat}

Instructions

- Faites une déclaration.

- Demandez à des volontaires de donner leur avis.

- Demandez à 2/3 volontaires de présenter leurs points de vue.

- Demandez à 2/3 volontaires avec des points de vue opposés de présenter leurs points de vue.

- Présentez les points de vue. 


\section{Groupe 4 : Narration}

Instructions

- Demandez à un volontaire de votre groupe de raconter une histoire simple et claire mettant en évidence un ou deux points.

- L'histoire doit être pertinente pour la vie des participantes.

- L'histoire doit être intéressante.

Puis discutez brièvement de l'histoire.

\section{PARTIE II : ENSEIGNEMENT PARTICIPATIF}

\section{Objectif}

Présenter et familiariser les mentores avec les outils à utiliser pendant le programme et fournir un guide étape par étape pour l'utilisation du programme

Durée : 60 minutes au total

Matériel : Flip chart, marqueur, programme (1 exemplaire pour chaque participante)

Processus : Les groupes font des présentations, puis l'animatrice explique après la présentation de chaque groupe

Durée : 30 minutes

Processus : L'animatrice présente le programme d'études sur la santé et les compétences de vie et guide les participantes à travers le programme étape par étape.

Durée : 30 minutes

Document à distribuer : Méthodes d'enseignement participatif : Chacune des méthodes d'enseignement peut être utilisée pour enseigner les compétences de vie. 


\section{LE RÔLE DE LA MENTORE DANS L’ENSEIGNEMENT ET L'APPRENTISSAGE}

Cette activité, à utiliser lors d'une formation d'introduction de la mentore, comprend des exercices sur le rôle et le style d'enseignement de la mentore, et une discussion sur les techniques d'apprentissage participatif adaptées à l'âge. Les formateurs trouvent que les adolescentes répondent plus favorablement lorsque la formation est de nature participative. Le format didactique traditionnel dans lequel les formateurs enseignent et répondent aux questions des étudiants a une valeur, en particulier pour transmettre des connaissances factuelles, telles que la manière dont le VIH est transmis ou le fonctionnement des méthodes contraceptives. Cependant, les formats de formation dans lesquels les participantes sont invitées à apporter leurs idées et leurs connaissances et à s'appuyer sur leur propre expérience semblent générer une participation plus enthousiaste et une plus grande rétention du sujet.

\section{EXERCICE 1 : LE RÔLE DU FORMATEUR}

Résultats attendus : Les mentores réfléchissent à leur rôle de formateur pour les adolescentes dans leurs espaces sûrs

Matériel nécessaire : Flip chart, marqueur, stylo

\section{Instructions}

- Demandez aux mentores d'énumérer les rôles que jouent les animateurs au cours d'un atelier.

- Demandez-leur de décrire ce que chaque rôle implique.

- Comparez leurs réponses avec ces rôles et tâches :

- Expert : Le formateur transmet ses connaissances et ses compétences, répond aux questions ou promet d'obtenir des informations pour les participantes plus tard, dissipe les idées fausses.

- Agent socialisant : Le formateur s'efforce de partager des valeurs et des idéaux avec les stagiaires, par exemple, les adolescents et les adultes devraient traiter leurs pairs féminins et masculins comme des égaux et prendre la responsabilité de promouvoir une sexualité saine.

- Facilitateur : Le formateur aide l'apprentissage à se faire à partir de l'expérience des participantes.

- Mentionnez que les formateurs ont la responsabilité de faciliter différents types d'apprentissages. Ceux-ci peuvent être résumés comme suit :

- Apprentissage du sujet; par exemple, qu'est-ce que le genre?

- Apprendre la relation entre le sujet et la vie réelle; par exemple, comment le genre affecte-t-il les relations des adolescents garçons et filles?

- Apprendre à appliquer les connaissances acquises; par exemple, comment les adolescents peuvent-ils utiliser leur sensibilité au genre pour reconnaître et éviter les risques pour la santé? 


\section{EXERCICE 2 : QU’EST-CE QUI REND L’APPRENTISSAGE AGRÉABLE?}

Résultats attendus : Les mentores sont capables d'identifier les facteurs qui ont rendu l'apprentissage agréable.

Matériel nécessaire : Feuilles de papier, crayons ou marqueurs, grille sur une grande feuille de papier postée à l'avant de la salle (un flip chart peut être utilisé).

\section{Instructions}

- Demandez aux participantes de dessiner deux images : l'une montrant comment elles ont appris à l'école primaire et l'autre comment elles ont appris au cours d'un atelier agréable plus tard dans la vie.

- Dessinez une grille sur un flip chart comme indiqué ci-dessous, en laissant les cases sous les en-têtes de deuxième et troisième colonnes vides.

- Invitez des participantes à montrer leurs images et à dire ce qu'elles représentent; demandez-leur de dire ce qu'elles ont aimé et ce qu'elles n'ont pas aimé des expériences qu'elles ont tirées de leurs images.

- Remplissez la grille en plaçant la déclaration des participantes sur ce qu'elles ont aimé et ce qu'elles n'aimaient pas dans les cases appropriées.

- Résumez les résultats, en mentionnant les points indiqués dans la grille :

\begin{tabular}{|l|l|l|}
\hline \multicolumn{1}{|c|}{ Des questions } & \multicolumn{1}{c|}{ Apprentissage à l'école primaire } & \multicolumn{1}{c|}{ Autre apprentissage } \\
\hline $\begin{array}{l}\text { Quel est le rôle de } \\
\text { l'apprenant? }\end{array}$ & $\begin{array}{l}\text { Passif; recevoir des informations; suivre } \\
\text { les instructions }\end{array}$ & $\begin{array}{l}\text { Actif; participatif; responsabilité } \\
\text { de l'apprentissage; interdépendant }\end{array}$ \\
\hline $\begin{array}{l}\text { Quelle est la motivation } \\
\text { pour apprendre? }\end{array}$ & $\begin{array}{l}\text { Bonnes notes; ne pas avoir d'ennuis; } \\
\text { châtiment; aucun avantage immédiat } \\
\text { observé; externe : la société dicte }\end{array}$ & $\begin{array}{l}\text { Apprendre de soi-même; } \\
\text { l'apprenant voit un avantage } \\
\text { immédiat; motivation interne }\end{array}$ \\
\hline Qui choisit le contenu? & $\begin{array}{l}\text { Prof ou conseil scolaire; pas de } \\
\text { choix de l'apprenant }\end{array}$ & $\begin{array}{l}\text { Intérêts propres; problèmes } \\
\text { de la vie }\end{array}$ \\
\hline $\begin{array}{l}\text { Quel est le focus de la } \\
\text { méthode? }\end{array}$ & $\begin{array}{l}\text { Apprentissage par cœur; communi- } \\
\text { cation unidirectionnelle; individuel }\end{array}$ & $\begin{array}{l}\text { Expérimental; des groupes } \\
\text { partagent leurs connaissances }\end{array}$ \\
\hline
\end{tabular}

\section{EXERCICE 3 : TRAVAIL DE GROUPE}

Résultats attendus : Les mentores réfléchissent à des techniques de facilitation adaptées à des cohortes d'âge particulières (par exemple, 10 à 14 ans et 15 à 19 ans), en groupes.

\section{Instructions}

- Répartissez les mentores en deux groupes, l'un représentant le groupe d'âge de 10 à 14 ans et l'autre représentant le groupe d'âge de 15 à 19 ans.

- Demandez à chaque groupe de réfléchir et d'énumérer les techniques de facilitation adaptées au groupe d'âge qui leur est assigné.

- En tenant compte de ce qu'elles ont énuméré, les groupes doivent répondre aux questions suivantes sur la manière dont elles peuvent contribuer à une expérience d'apprentissage agréable sur les adolescentes dans leurs espaces sûrs:

- Quelles sont les raisons pour lesquelles les adolescentes apprennent?

- Quel est votre rôle en tant que mentore dans leur expérience d'apprentissage?

- Comment décririez-vous l'environnement d'apprentissage dans l'espace sûr?

- Comment sauriez-vous qu'un apprentissage a bien eu lieu?

- Demandez à chaque groupe de préparer des "astuces de formation » pour les autres en fonction de leurs réponses.

- Tapez ou écrivez sur des fiches les «astuces de formation » et donnez-les à toutes les participantes le lendemain pendant qu'elles se préparent pour les séances pratiques. 


\section{PRÉSENTATION DE LA FORMATION : INTRODUCTION AU COUNSELING}

On attend souvent des mentores qu'elles fassent du counseling dans le cadre de leur travail avec les AJF. Les objectifs de cette formation sont de décrire les buts et les principes du counseling, d'enseigner aux mentores l'utilisation des compétences en matière de counseling et de fournir la possibilité de mettre en pratique ces compétences. Le personnel du programme doit reconnaître que la plupart des mentores ont été confrontées (ou sont actuellement confrontées) à des défis similaires pour les AJF du programme, et le personnel doit soutenir adéquatement les mentores pour répondre à la fois aux défis des filles et à leur propre bien-être psychologique. Un soutien peut être fourni lors des vérifications hebdomadaires et mensuelles, bien que certaines mentores aient besoin de contacts plus réguliers avec les superviseurs ou, dans certains cas, d'une aide professionnelle dédiée. Les programmes devraient fournir aux mentores des informations sur les sources de soutien psychosocial pour elles-mêmes et pour les participantes au programme.

Cette présentation couvre les attitudes d'un bon conseiller, les environnements du counseling

\section{LES PRINCIPES DU COUNSELING DE BASE}

Le conseil vise à aider les personnes à mieux faire face aux situations auxquelles elles sont confrontées. Cela implique d'aider les individus à faire face à leurs émotions et leurs sentiments et à les aider à faire des choix et des décisions positifs.

Diapositive 1

\section{LES PRINCIPES DU COUNSELING DE BASE}

\section{Faire cela implique:}

- Fournir des informations correctes;

- Aider l'individu à prendre des décisions éclairées (autonomie);

- Aider le client à reconnaître et à développer ses forces (autonomisation);

- Aider le client à développer une attitude positive ; et,

- Entretenir une relation professionnelle.

Diapositive 3
LES PRINCIPES DU COUNSELING DE BASE

Faire cela implique:

- Établir une relation de confiance ;

- Aider la fille conseillée à raconter son histoire ;

- Écouter attentivement ;

- Respecter la fille conseillée ;

- Ne pas porter de jugement ;

- Assurer la confidentialité.

Diapositive 2

\section{LES PRINCIPES DU COUNSELING DE BASE}

IL N'IMPLIQUE PAS:

- Prendre des décisions pour la fille conseillée ;

- Juger, interroger, blâmer, prêcher, donner des conférences ou argumenter ;

- Faire des promesses qu'on ne peut pas tenir ;

- Permettre aux filles de devenir dépendantes de vous.

Le counseling est une approche pour aider les gens pendant et juste après une pandémie, pour réduire la détresse initiale et pour encourager un fonctionnement adaptatif à court et à long terme.

Diapositive 4 


\section{ATTITUDES D'UN BON CONSEILLER}

- $\quad$ Authenticité - être honnête avec les filles et avec soi-même. Cependant, pour être honnête, ne pas nuire aux filles émotionnellement/psychologiquement

- Empathie - vous mettre dans la situation de la fille conseillée tout en restant objective ; être sans jugement, sensible et compréhensive.

\section{COMPÉTENCES ASSISTANTES}

\section{Langage corporel et présence émotionnelle}

- Soyez attentive aux gestes que vous faites et à ceux de la jeune fille conseillée. Votre corps est un moyen de communication avec la fille tandis que le corps de la fille est une source d'information.

- $\quad$ Soyez attentive à la fille conseillée à la fois psychologiquement et physiquement.

\section{COMPÉTENCES ASSISTANTES (suite)}

\section{Questions ouvertes et approfondies}

- Les questions ouvertes (qui, quoi, où, quand, comment) et des questions d'approfondissement invitent des réponses de plus d'un ou deux mots. Celles-ci peuvent être utilisées pour recueillir des informations, accroître la clarté, stimuler la réflexion ou créer une discussion.

\section{ATTITUDES D'UN BON CONSEILLER}

- Acceptation - valoriser tous les aspects de la fille conseillée, que sa situation soit mauvaise ou bonne (regard positif inconditionnel); accepter inconditionnellement indépendamment du sexe, de l'âge, du handicap, du statut économique, de la race, etc.

Diapositive 6

\section{COMPÉTENCES ASSISTANTES (suite)}

\section{Être compatisante}

- L'empathie signifie se mettre dans la situation de la fille conseillée tout en restant objectif. L'empathie exige que le conseiller ne porte pas de jugement, soit sensible et compréhensive.

\section{COMPÉTENCES ASSISTANTES (suite)}

\section{Écoute active}

- Écoutez attentivement la jeune fille au lieu de penser à ce que vous allez dire ensuite.

- Asseyez-vous confortablement. Évitez les mouvements distrayants. Regardez directement la fille quand elle parle, pas vos papiers ou par la fenêtre.

- De temps en temps, répétez dans vos mots ce que vous avez entendu. C'est ce qu'on appelle la " paraphrase ». Alors vous et la jeune fille conseillée savez si vous avez compris. 


\section{PRÉSENTATION DE LA FORMATION : INTRODUCTION AU COUNSELING (suite)}

\section{COMPÉTENCES ASSISTANTES (suite)}

\section{Résumer}

- Cela peut être fait au début, en continu et à la fin de la session, afin que vous puissiez toutes les deux vous mettre d'accord sur ce qui a été discuté et ce que vous ferez toutes les deux après ce départ.

- Mettez en évidence et clarifiez les problèmes afin que la fille conseillée puisse avoir une vue d'ensemble.

- Identifiez les solutions possibles qui ont été discutées.

- Demandez à la fille conseillée ce qu'elle pense de ce qui a été discuté.

Diapositive 11

\section{SOUS-COMPÉTENCES DE PARTICIPATION}

Asseyez-vous carrément : faites face à la fille conseillée, à moins que cela ne soit considéré comme impoli (dans certaines cultures)

Posture ouverte : évitez les bras croisés et se pencher en arrière.

Penchez-vous légèrement vers la fille conseillée : montre de l'intérêt pour ses problèmes.

Contact visuel direct avec la fille conseillée : cela fait ressortir la vérité.

Soyez d'humeur détendue en tant que conseiller

\section{PROCÉDURE DE COUNSELING}

1. Créer un rapport

- Bienvenue et offrez une place

- Présentations

- Assurez que la fille soit informée de la confidentialité, des délais et des responsabilités

- Procédure/type de conseil : individuel ou en groupe

2. Demandez à la fille qu'on conseille de dire pourquoi elle est venue.

3. Utilisez les compétences de conseil - vers la fin : fixez des objectifs et résumer.
Diapositive 12

\section{TRAVAUX PRATIQUES}

Par deux, présentez un cas de counseling et jouez les compétences de counseling suivantes

1. Compétences d'empathie et d'écoute

2. Compétences ouvertes et approfondies

3. Paraphrasez réfléchissez, reformulez les compétences

4. Compétence en définition et synthèse des objectifs 


\section{SESSION DE FORMATION DES MENTORES SUR LA VIOLENCE BASÉE SUR LE GENRE ET LES DROITS HUMAINS}

Cette session de formation d'une heure et demie couvre les concepts clés et les questions fondamentales qui sous-tendent la violence basée sur le genre (VBG). Son objectif est d'augmenter la capacité des participantes à discuter des concepts clés de manière à être compris par la communauté ; identifier la relation entre l'abus de pouvoir et la VBG; comprendre que le terme «violence » dans le contexte de la VBG signifie l'utilisation d'un certain type de force, qui peut inclure ou non la force physique ; comprendre la signification du «consentement éclairé » et sa relation avec la VBG; et comprendre la relation entre les droits humains et la VBG.

\section{La violence basée sur le genre et les droits humains}

\section{Durée : $1 \mathrm{~h} 30$}

\section{Objectifs de la formation :}

- Aider les participantes à comprendre et à décrire les concepts clés et les problèmes de base sous-tendant toutes les formes de VBG

- Accroítre la capacité des participantes à discuter des concepts clés de manière à être bien compris par la communauté

- Identifier la relation entre l'abus de pouvoir et la VBG

- Comprendre que le terme «violence» dans le contexte de la VBG signifie l'utilisation d'un certain type de force, qui peut inclure ou non la force physique

- Comprendre la signification de « consentement éclairé » et sa relation avec la VBG

- Comprendre la relation entre les droits humains et la VBG

\section{Aperçu :}

Cette session explore la signification de trois concepts importants derrière le terme VBG. Les trois concepts sont combinés dans cette session, mais ils sont présentés et discutés un par un, pour continuer à développer la compréhension des participantes soigneusement, pas à pas. Deux exemples de cas sont utilisés pour la discussion de groupe pour renforcer l'apprentissage de ces concepts.

Matériel : Flipchart, marqueurs

Type de session : Discussion, activités de groupe

\section{Processus :}

Le pouvoir (10 minutes)

1. Sur le flip chart, écrivez le mot POUVOIR.

2. Demandez au groupe «qu'est-ce qui donne du pouvoir à quelqu'un? " Et écrivez les réponses sur le flip chart.

3. Discutez de divers types de pouvoir. Demandez des exemples (sans nom) de personnes qui ont du pouvoir dans le monde, dans la communauté.

4. Expliquez que la VBG consiste à abuser du pouvoir, qu'il soit réel ou perçu; la victime d'abus croit que le pouvoir est réel.

5. Collez la feuille du flip chart sur le pouvoir sur le mur à proximité, de manière visible pour qu'au besoin plus tard, on puisse se référer.

Violence/recours à la force (10 minutes)

1. Sur une nouvelle feuille vierge, écrivez le mot VIOLENCE.

2. Demandez à chaque participante de prendre un morceau de papier et d'écrire deux mots ou phrases pour décrire ce que nous entendons par "violence» lorsque nous parlons de VBG. II s'agit d'une activité individuelle, et non un travail de groupe. Prévoyez quelques instants pour que chacune puisse écrire ses deux mots.

3. Faites le tour de la pièce, une par une, en demandant à chaque personne de donner un mot/une phrase qu'elle a écrite. Mettez le mot sur le flip chart. Continuez à faire le tour de la salle jusqu'à ce que vous ayez le mot de tout le monde sur le flip chart. C'est un exercice qui prend peu de temps.

4. Les participantes donnent généralement une combinaison d'exemples de types de violence ainsi que quelques définitions du mot «violence ». 


\section{SESSION DE FORMATION DES MENTORES SUR LA VIOLENCE BASÉE SUR LE GENRE ET LES DROITS HUMAINS (suite)}

5. Éloignez-vous du flip chart et animez une brève discussion pour faire ressortir les principaux points de discussion, biffer les mots ou les phrases dont les participantes conviennent qu'ils ne font pas partie de la liste.

- En haut du tableau, écrivez «Utilisation de la force » à côté de «Violence ». Devrait ressembler à ceci : VIOLENCE/UTILISATION DE LA FORCE.

- Résumez en expliquant que la violence dans ce contexte implique l'utilisation d'un certain type de force, réelle et implicite. C'est un élément clé pour définir ce que nous entendons lorsque nous parlons de VBG.

- Collez la feuille sur la violence sur le mur près du flip chart «Pouvoir », où ils peuvent tous les deux être vus et mentionnés plus tard dans la session.

Consentement éclairé ( 5 minutes)

1. Sur la nouvelle feuille vierge, écrivez le mot CONSENTEMENT.

2. Demandez aux participantes ce que signifie le consentement pour elles. Écrivez leurs réponses sur le flip chart. Discutez de leurs réponses et assurez-vous de souligner deux éléments nécessaires du consentement qui sont «éclairé » et « volontaire».

3. Résumez la session en montrant les trois feuilles : Pouvoir, violence/usage de la force, consentement. Passez rapidement en revue les principaux points de chacun de ces concepts clés. Lisez l'exemple suivant aux participantes. Posez ensuite chaque question et discutez:

«Dans une famille très traditionnelle et patriarcale, le père d'une jeune fille de 16 ans lui dit qu'il lui a arrangé un mariage avec un certain homme. La fille ne connaît pas très bien l'homme, il est beaucoup plus âgé qu'elle, mais elle accepte de l'épouser $»$.
- Pensez-vous que ce genre de situation pourrait se produire?

- A-t-elle donné son consentement éclairé à ce mariage?

- Y a-t-il eu un recours à la force lors de cet incident?

- Qui est le plus puissant dans cet exemple : le père ou la fille?

- Quel genre de pouvoir a ce père?

4. Quel genre de pouvoir a la fille?

5. Comment le pouvoir est-il lié au choix dans cet exemple?

\section{Droits humains}

Aperçu : Tous les actes de VBG constituent une violation des droits humains fondamentaux. La session explore brièvement les droits humains dans le contexte de la VBG, qui est un autre concept ou principe de base qui conduira les participantes à une compréhension claire de la signification de la VBG.

Objectif : Comprendre la relation entre les droits humains et la VBG

Matériel : Flipchart, marqueurs

Type de session : Discussion

\section{Processus :}

1. Écrivez DROITS HUMAINS sur le flip chart. Demandez aux participantes qui a des droits humains et écrivez leurs réponses sur le flip chart. Discutez du concept selon lequel chacun a des droits humains. Demandez aux répondants qui ou qu'est-ce qui accorde les droits humains. Discutez du concept selon lequel personne ne doit vous accorder ces droits parce que vous les avez automatiquement dès la naissance.

2. Demandez aux participantes des exemples de droits humains et écrivez leurs réponses sur le flip chart. 


\section{Points de discussion clé :}

1. Les droits humains sont universels, inaliénables, indivisibles, interconnectés et interdépendants.

2. Chacun a droit à tous les droits et libertés, sans distinction d'aucune sorte, telle que la race, la couleur, le sexe, la langue, la religion, l'opinion politique, etc.

3. La prévention et la réponse à la VBG sont directement liées à la protection des droits humains.

4. Les actes de VBG violent un certain nombre de principes des droits humains consacrés dans les instruments internationaux. Ceux-ci incluent :

- Le droit à la vie, à la liberté et à la sécurité de la personne

- Le droit au plus haut niveau de santé physique et mentale

- Le droit de ne pas être soumis à la torture, au viol ou à des peines ou traitements cruels, inhumains ou dégradants

- Le droit à la liberté d'opinion et d'expression, à l'éducation, à la sécurité sociale et au développement personnel

\section{Conclusion de la session :}

Le facilitateur doit mentionner que :

1. Les auteurs de VBG peuvent avoir un pouvoir "réel ou "perçu» (par exemple, social : pression des pairs et intimidation; basé sur le sexe : les hommes occupent généralement une position plus puissante que les femmes).

2. Le pouvoir est directement lié au choix. Plus on a le pouvoir, plus on a le choix. Moins on a le pouvoir, moins on a le choix.
3. La VBG implique l'abus de pouvoir. Dans les relations de pouvoir inégales, les individus peuvent être exploités ou maltraités.

4. Est-ce que toutes les personnes qui ont du pouvoir abusent de leur pouvoir? NON.

5. La «force » peut être de nature physique, émotionnelle, sociale ou économique.

6. Le consentement signifie dire «OUI» d'accepter quelque chose. Le consentement éclairé signifie faire un choix éclairé librement et volontairement par des personnes dans une relation de pouvoir égal.

7. Les enfants (de moins de 18 ans) sont réputés incapables de donner leur consentement éclairé pour des actes tels que le mariage, les relations sexuelles, etc. 


\section{SESSION DE FORMATION DES MENTORES SUR LE TRAVAIL AVEC LES FILLES VIVANT AVEC UN HANDICAP}

\section{Objectifs}

- Sensibiliser au handicap, aux droits et aux problèmes auxquels sont confrontées les filles handicapées

- Renforcer les compétences des participantes en matière d'identification du handicap et de travail avec les filles handicapées dans des espaces sûrs

\section{Étapes}

\section{Introduction}

Introduisez la session sur le handicap et demandez des volontaires pour proposer des activités de réveil lié ou approprié au sujet

\section{Travaux de groupe}

1. Formez des groupes avec les participantes, idéalement, des groupes de quatre.

2. Laissez-les discuter de ce qui suit :

- Qu'entendez-vous par le mot «handicap»?

- Quels types de handicaps connaissezvous?/avez-vous vu ou entendu parler?

- Quels sont les défis auxquels nous sommes confrontées ou que nous sommes susceptibles d'être confrontées dans notre travail avec les filles handicapées dans les espaces sûrs?

- Comment pouvons-nous les aborder?

- Comment percevez-vous les personnes vivant avec un handicap?

- Faites une plénière une fois les discussions de groupe ont été conclues. Posez des questions et clarifiez si nécessaire.

\section{Présentation :}

- Lisez «L'histoire de Sarah » à vos participantes et ensuite, en vous basant sur I'histoire, discutez et réfléchissez à la discrimination contre les filles handicapées et comment elle peut être surmontée.

- Concluez en demandant aux participantes de partager ce qu'elles ont appris et également si elles connaissent des femmes/filles modèles vivant avec un handicap.

\section{L'histoire de Sarah : La discrimination à l'égard des filles handicapées}

Sarah est une fille du programme de l'espace sûr. Elle assiste régulièrement à des réunions et est avide d'apprendre. Son défi est que sa parole est altérée et chaque fois qu'elle parle pendant la session, ses amis se moquent d'elle. Cela a perturbé sa participation et son assiduité de l'espace sûr.

\section{Qu'est-ce c'est que la discrimination ?}

Lorsqu'une personne est traitée injustement en raison d'une stigmatisation, c'est-à-dire qu'elle est traitée de manière injuste en raison de perceptions ou de préjugés à son égard.

\section{Comment la discrimination est-elle présentée dans l'histoire de Sarah?}

- Quelqu'un voudrait-elle partager des récits de ce qu'elles ont vécu dans leur espace sûr qui démontre la discrimination à l'égard des filles handicapées?

- Quelles sont les conséquences possibles de la stigmatisation et de la discrimination à l'égard des filles handicapées?

Au nombre des réponses possibles figurent : absentéisme, faible estime de soi, peur de s'associer avec d'autres personnes, ou isolement.

- Comment les mentores peuvent-elles faire face à la discrimination contre les filles handicapées dans leurs espaces sûrs? 


\section{PROGRAMME DE LA FORMATION DE RECYCLAGE DES MENTORES}

Une formation ne suffit généralement pas aux mentores pour avoir confiance en leur capacité à se souvenir des concepts clés et à fournir du contenu. Des réunions régulières sont importantes pour la réflexion, la discussion et les occasions de pratiquer la facilitation. Il peut également être utile de réunir des mentores pour une version simplifiée d'une formation après quelques mois d'expérience en tant que mentor.

Ci-dessous, un exemple de programme d'une formation de recyclage de 4,5 jours avec 35 mentores participantes. Il détaille une formation de recyclage qui vise à : obtenir du feedback des mentores sur leur expérience de fourniture d'informations sur les curriculums sur les compétences de vie, la santé et droits sexuels et reproductifs et l'éducation financière; revoir et améliorer les compétences des mentores dans les méthodes de facilitation interactives; permettre aux mentores d'acquérir des informations précises sur les compétences de vie, la santé et droits sexuels et reproductifs et la gestion financière; et permettre aux mentores d'acquérir plus d'expérience dans l'offre de conseils et de soutien social aux filles pendant les sessions d'espace sûr.

Période de formation :

Date:

Site:

Participantes (mentores):

Lieu:

Facilitateurs: un coordinateur de programme, deux coordinateurs de site

\section{Objectifs:}

L'objectif général de l'atelier de la formation de recyclage des mentores des espaces sûrs est destiné au soutien continu et au renforcement des capacités des mentores afin de faciliter la mise en œuvre des espaces sûrs et de fournir efficacement des curriculums en santé, compétence de vie et d'éducation financière aux filles du programme.

Les objectifs spécifiques de l'atelier sont:

- Obtenir du feedback des mentores sur leur expérience dans la mise en œuvre du curriculum.

- Réviser et améliorer les compétences des mentores en facilitant une gamme de méthodologies interactives pendant les sessions sur l'espace sûr.

- Acquérir (pour les mentores) des informations précises sur les compétences de vie, la santé et droits sexuels et reproductifs et la gestion financière.

- Acquérir (pour les mentores) plus d'expérience dans l'offre de conseils et de soutien social aux filles lors des sessions d'espace sûr. 


\section{PROGRAMME DE LA FORMATION DE RECYCLAGE DES MENTORES (suite)}

\section{Jour 1}

\begin{tabular}{|c|c|c|c|}
\hline Horaires & Session & Objectifs & Facilitateur \\
\hline $16.00-16.10$ & $\begin{array}{l}\text { Bienvenue, présentations des } \\
\text { participantes/brise-glace }\end{array}$ & & \\
\hline $16.10-16.55$ & Outil - sondage des mentores & - Évaluer le niveau de connaissances des participantes & \\
\hline $16.55-17.05$ & Objectifs & - Clarifier les objectifs de l'atelier & \\
\hline $17.05-17.20$ & Règles de base & - Convenir des règles de base de l'atelier & \\
\hline $17.20-17.35$ & Attentes des participantes & - Partager les attentes pour la formation de recyclage & \\
\hline $17.35-18.00$ & Présentation & $\begin{array}{l}\text { - Rafraîchir les mentores du programme } \\
\text { - Rafraî́chir sur les objectifs du programme } \\
\text { - Rafraî́chir sur le volet évaluation du programme }\end{array}$ & \\
\hline $18.00-19.30$ & $\begin{array}{l}\text { Session de } \\
\text { feedback }\end{array}$ & $\begin{array}{l}\text { - Recueillir le feedback des mentores sur leur } \\
\text { expérience dans la mise en œuvre des espaces sûrs } \\
\text { - Partager les défis } \\
\text { - Réfléchir aux solutions possibles aux défis } \\
\text { rencontrés }\end{array}$ & \\
\hline 19:30 & & SYNTHESE, PAUSE DÎNER & \\
\hline
\end{tabular}

Jour 2

\begin{tabular}{|c|c|c|c|}
\hline Horaires & Session & Objectifs & Facilitateur \\
\hline $8.00-8.15$ & Activité de réveil/Ménage & $\begin{array}{l}\text { - Offrir aux participantes l'expérience des activités } \\
\text { de réveil du manuel Energizer/d'autres activités } \\
\text { de réveil suggérées }\end{array}$ & \\
\hline $8.15-8.30$ & Feedback sur le jour 1 & - Faire une évaluation continue de l'atelier & \\
\hline $8.30-10.30$ & $\begin{array}{l}\text { Session d'information } \\
\text { Développement des } \\
\text { compétences }\end{array}$ & $\begin{array}{l}\text { - Recevoir une mise à jour sur leurs compétences clés } \\
\text { en animation } \\
\text { - Comprendre comment appliquer différentes } \\
\text { compétences pour faciliter les espaces sûrs } \\
\text { - Introduire des techniques pour réussir à impliquer les } \\
\text { filles dans la session } \\
\text { - Brainstormings sur les techniques adaptées à des } \\
\text { tranches d'âge particulières en petits groupes }\end{array}$ & \\
\hline $10.30-11.00$ & \multicolumn{2}{|r|}{ PAUSE CAFÉ } & \\
\hline $11.00-13.00$ & $\begin{array}{l}\text { Session d'information } \\
\text { Clarification des valeurs de } \\
\text { la santé et droits sexuels et } \\
\text { reproductifs (SDSR) des } \\
\text { adolescentes }\end{array}$ & $\begin{array}{l}\text { - Aborder les connaissances, les attitudes et les } \\
\text { comportements des mentores en matière de } \\
\text { SDSR pour les adolescentes } \\
\text { - Donner un aperçu de la réalité sur le terrain pour } \\
\text { les adolescentes et la SDSR } \\
\text { - Améliorer la sensibilisation aux droits SDSR des } \\
\text { adolescentes_[Pays] }\end{array}$ & \\
\hline $13.00-14.00$ & \multicolumn{2}{|r|}{ PAUSE DÉJEUNER } & \\
\hline
\end{tabular}




\begin{tabular}{|c|c|c|}
\hline $14.00-16.00$ & $\begin{array}{l}\text { Session d'information } \\
\text { Infections sexuellement } \\
\text { transmissible (IST) et } \\
\text { rapports sexuels protégés }\end{array}$ & $\begin{array}{l}\text { À la fin de cette session, les participantes devraient } \\
\text { être capables de : } \\
\text { - Expliquer la signification des IST } \\
\text { - Décrire quatre symptômes courants des IST } \\
\text { - Éxpliquer la relation entre les IST et l'infection à VIH } \\
\text { - Énumérer au moins quatre idées fausses sur les IST } \\
\text { - Expliquer les conséquences des IST si elles ne sont } \\
\text { pas traitées }\end{array}$ \\
\hline $16.00-16.30$ & \multicolumn{2}{|r|}{ PAUSE CAFÉ } \\
\hline $16.30-17.30$ & $\begin{array}{l}\text { Rappel de l'approche } \\
\text { "Ancrer, Ajouter, Appliquer, } \\
\text { s'Éloigner », programme } \\
\text { d'approche (qui impliquent } \\
\text { avec succès les cohortes } \\
\text { d'âge différentes) } \\
\text { Séance d'affectation : } \\
\text { Les participantes se voient } \\
\text { assigner les sujets du } \\
\text { programme d'études }\end{array}$ & $\begin{array}{l}\text { - Les participantes comprennent l'approche et } \\
\text { apprennent à les appliquer lors de l'exécution des } \\
\text { sessions du curriculum } \\
\text { - Les participantes sont divisées en deux groupes par } \\
\text { âge (10-14 et 15-19) et chaque groupe est attribué } \\
\text { une session pour préparer et présenter } \\
\text { - Les présentations devraient se concentrer sur } \\
\text { l'application de techniques sur la façon de réussir à } \\
\text { impliquer les différentes cohortes d'âges lors des } \\
\text { sessions d'espace sûr }\end{array}$ \\
\hline $17.30-18.30$ & $\begin{array}{l}\text { Séances préparatoires aux } \\
\text { présentations de groupe }\end{array}$ & $\begin{array}{l}\text { - Les participantes se regroupent pour préparer les } \\
\text { présentations }\end{array}$ \\
\hline $18.30-19.30$ & $\begin{array}{l}\text { Équipe } 1 \text { : Groupe d'âge 10- } \\
14 \text { ans (choisir une session } \\
\text { dans le programme) } \\
\text { Feedback des groupes de } \\
\text { sessions pratiques }\end{array}$ & $\begin{array}{l}\text { - Les participantes mettent en pratique leurs } \\
\text { compétences de facilitation et utilisent des } \\
\text { techniques qui impliquent avec succès les groupes } \\
\text { d'âge } 10-14 \text { ans } \\
\text { - Les participantes identifient les compétences de } \\
\text { facilitation utilisées pour faciliter efficacement } \\
\text { - Les participantes discutent des défis rencontrés } \\
\text { pendant la facilitation }\end{array}$ \\
\hline 19.30 & \multicolumn{2}{|r|}{ SYNTHĖSE, PAUSE DÎNER } \\
\hline
\end{tabular}

Jour 3

\begin{tabular}{|c|c|c|c|}
\hline Horaires & Session & Objectifs & Facilitateur \\
\hline $8.00-8.15$ & Activité de réveil/Ménage & $\begin{array}{l}\text { - Montrer aux participantes l'expérience des } \\
\text { activités de réveil du manuel/d'autres activités } \\
\text { énergisantes suggérées }\end{array}$ & \\
\hline $8.15-8.30$ & Feedback sur le jour 2 & - Fournir une évaluation continue de l'atelier & \\
\hline $8.30-10.30$ & $\begin{array}{l}\text { Séance de discussion } \\
\text { Examen du feedback et } \\
\text { des expériences en } \\
\text { éducation financière dans } \\
\text { des espaces sûrs; } \\
\text { obstacles et découvertes } \\
\text { positives }\end{array}$ & $\begin{array}{l}\text { - Les participantes partagent et discutent des } \\
\text { défis et des expériences positives pour faciliter } \\
\text { l'éducation financière dans des espaces sûrs }\end{array}$ & \\
\hline $10.30-11.00$ & \multicolumn{2}{|r|}{ PAUSE CAFÉ } & \\
\hline $11.00-13.00$ & $\begin{array}{l}\text { Thème de formation : } \\
\text { Explorer les options pour } \\
\text { optimiser l'épargne et } \\
\text { l'utilisation de comptes } \\
\text { grâce à des espaces sûrs (si } \\
\text { les groupes d'épargne font } \\
\text { partie du programme) }\end{array}$ & $\begin{array}{l}\text { - Les participantes acquièrent des compétences } \\
\text { pratiques pour soutenir les pratiques d'épargne } \\
\text { dans des espaces sûrs } \\
\text { - Les participantes explorent les options } \\
\text { d'implication des filles dans des stratégies } \\
\text { d'utilisation des comptes, y compris des } \\
\text { messages réguliers }\end{array}$ & \\
\hline $13.00-14.00$ & & PAUSE DÉJEUNER & \\
\hline
\end{tabular}




\section{PROGRAMME DE LA FORMATION DE RECYCLAGE DES MENTORES (suite)}

\begin{tabular}{|c|c|c|}
\hline $14.00-16.00$ & $\begin{array}{l}\text { Thème de formation } \\
\text { Jeu de rôle pour résoudre } \\
\text { les conflits } \\
\text { Session d'affectation : les } \\
\text { participantes reçoivent des } \\
\text { thèmes tirés du curriculum }\end{array}$ & $\begin{array}{l}\text { - Les participantes acquièrent des compétences } \\
\text { dans l'utilisation des jeux de rôle pour accroître } \\
\text { la compréhension des filles de l'argent } \\
\text { La présentation se concentrera sur l'utilisation } \\
\text { du programme de l'approche pour accroître les } \\
\text { compétences de facilitation et familiariser les } \\
\text { participantes avec les thèmes du curriculum. }\end{array}$ \\
\hline $16.00-16.30$ & \multicolumn{2}{|r|}{ PAUSE CAFÉ } \\
\hline $16.30-18.30$ & $\begin{array}{l}\text { Session préparatoire à la } \\
\text { présentation de groupe }\end{array}$ & $\begin{array}{l}\text { Les participantes vont dans leurs groupes pour } \\
\text { se préparer aux présentations }\end{array}$ \\
\hline $18.30-19.30$ & $\begin{array}{l}\text { Feedback des groupes de } \\
\text { sessions pratiques }\end{array}$ & $\begin{array}{l}\text { Les participantes identifient les compétences de } \\
\text { facilitation utilisées pour faciliter efficacement }\end{array}$ \\
\hline 19.30 & \multicolumn{2}{|r|}{ SYNTHĖSE, PAUSE DÎNER } \\
\hline
\end{tabular}

Jour 4

\begin{tabular}{|c|c|c|c|}
\hline Horaires & Session & Objectifs & Facilitateur \\
\hline $8.00-8.15$ & Activité de réveil/Ménage & $\begin{array}{l}\text { - Partager avec les participantes l'expérience des } \\
\text { activités de réveil du manuel Energizer/d'autres } \\
\text { activités de réveil suggérées }\end{array}$ & \\
\hline $8.15-8.30$ & Feedback sur le jour 3 & - Assurer une évaluation continue de l'atelier & \\
\hline $\begin{array}{l}8.30-9.00 \\
9.00-10.30\end{array}$ & $\begin{array}{l}\text { Compte d'épargne } \\
\text { Examen des bons de } \\
\text { santé }\end{array}$ & $\begin{array}{l}\text { - Passer en revue toutes les questions clés sur le } \\
\text { compte d'épargne (si cela fait partie du programme) } \\
\text { - Passer en revue les questions clés sur les bons de } \\
\text { santé (si cela fait partie de votre programme) }\end{array}$ & \\
\hline $10.30-11.00$ & & PAUSE CAFÉ & \\
\hline $11.00-13.00$ & $\begin{array}{l}\text { Séance d'information } \\
\text { Travailler avec des filles } \\
\text { handicapées }\end{array}$ & $\begin{array}{l}\text { - Identifier et traiter les questions des personnes } \\
\text { handicapées dans les groupes d'espaces sûrs } \\
\text { - Développer les compétences à travailler avec des } \\
\text { filles handicapées }\end{array}$ & \\
\hline $13.00-14.00$ & & PAUSE DÉJEUNER & \\
\hline $14.00-16.00$ & $\begin{array}{l}\text { Session d'information } \\
\text { Viol et VBG }\end{array}$ & $\begin{array}{l}\text { - Les participantes comprennent le viol, le viol par une } \\
\text { connaissance et l'inceste } \\
\text { - Les participantes comprennent les moyens de } \\
\text { prévenir et de traiter les abus sexuels et le viol } \\
\text { - Les participantes apprennent les droits de la SDSR }\end{array}$ & \\
\hline $16.00-16.30$ & & PAUSE CAFÉ & \\
\hline $16.30-18.30$ & $\begin{array}{l}\text { Session d'information } \\
\text { Offre de soutien et de } \\
\text { référence }\end{array}$ & $\begin{array}{l}\text { - Comprendre les aspects clés du soutien } \\
\text { psychosocial } \\
\text { - Identifier les signes avant-coureurs du besoin de } \\
\text { soutien psychosocial et de références } \\
\text { - Identifier les systèmes et les structures } \\
\text { communautaires de soutien et de référence } \\
\text { - Comprendre les rôles que les différents systèmes } \\
\text { communautaires identifiés peuvent jouer dans l'offre } \\
\text { de soutien }\end{array}$ & \\
\hline $18.30-19.30$ & $\begin{array}{l}\text { Équipe } 2: \text { tranche d'âge } \\
\text { de } 15 \text { à } 19 \text { ans (choisir } \\
\text { une session dans le } \\
\text { programme) } \\
\text { Feedback des travaux } \\
\text { de groupe des sessions } \\
\text { pratiques }\end{array}$ & $\begin{array}{l}\text { - Les participantes mettent en pratique leurs } \\
\text { compétences de facilitation et utilisent des } \\
\text { techniques qui impliquent avec succès les } \\
\text { groupes d'âge de } 10 \text { à } 14 \text { ans } \\
\text { - Les participantes identifient les compétences de } \\
\text { facilitation utilisées pour faciliter efficacement } \\
\text { - Les participantes discutent des défis }\end{array}$ & \\
\hline 19.30 & \multicolumn{2}{|r|}{ SYNTHĖSE, PAUSE DÎNER } & \\
\hline
\end{tabular}


Jour 5

\begin{tabular}{|c|c|c|c|}
\hline Horaires & Session & Objectifs & Facilitateur \\
\hline $8.00-8.15$ & $\begin{array}{l}\text { Activité de réveil/ } \\
\text { Rangement }\end{array}$ & $\begin{array}{l}\text { - Partager avec les participantes l'expérience des } \\
\text { activités de réveil du manuel Energizer/d'autres } \\
\text { activités de réveil suggérées }\end{array}$ & \\
\hline $8.15-8.30$ & Feedback sur le jour 4 & - Assurer une évaluation continue de l'atelier & \\
\hline $8.30-10.30$ & $\begin{array}{l}\text { Application de } \\
\text { Participation (si vous } \\
\text { utilisez une méthode } \\
\text { basée sur une } \\
\text { application pour } \\
\text { maintenir la } \\
\text { participation) }\end{array}$ & $\begin{array}{l}\text { - Reviser l'application } \\
\text { - Enseigner aux mentores comment télécharger un } \\
\text { nouveau formulaire } \\
\text { - Discuter des défis et réfléchir aux solutions } \\
\text { - Examiner les critères, y compris à quel moment une fille } \\
\text { est marquée comme présente au rencontre sur l'espace } \\
\text { sûr, en fonction de l'heure d'arrivée } \\
\text { - Participation aux séances de rattrapage }\end{array}$ & \\
\hline $10.30-11.00$ & \multicolumn{2}{|r|}{ PAUSE CAFÉ } & \\
\hline $11.00-13.00$ & Visites à domicile & $\begin{array}{l}\text { - Comprendre l'importance d'effectuer des visites à } \\
\text { domicile } \\
\text { - Comprendre comment structurer les visites à domicile } \\
\text { - Comprendre comment rendre compte des visites à } \\
\text { domicile } \\
\text { - Partager les défis et réfléchir aux solutions }\end{array}$ & \\
\hline $13.00-14.00$ & \multicolumn{2}{|r|}{ PAUSE DÉJEUNER } & \\
\hline $14.00-15.00$ & Suivi et évaluation & $\begin{array}{l}\text { - Revue des outils de suivi et d'évaluation } \\
\text { - Registres/Liste de présence } \\
\text { - Rapports } \\
\text { - Plans de cours } \\
\text { - Documentation des réussites } \\
\text { - Cycle du programme d'étude (ce qu'il faut faire une fois } \\
\text { terminé le premier cycle du curriculum) }\end{array}$ & \\
\hline $15.00-16.30$ & $\begin{array}{l}\text { Évaluation et } \\
\text { prochaines étapes }\end{array}$ & $\begin{array}{l}\text { - Résumer les réalisations de l'atelier } \\
\text { - Expliquer ce que l'on attend des mentores pour les } \\
\text { prochaines étapes }\end{array}$ & \\
\hline $16.30-17.00$ & $\begin{array}{l}\text { Questionnaire post- } \\
\text { test }\end{array}$ & $\begin{array}{l}\text { - Mesurer les niveaux de connaissances acquises par les } \\
\text { participantes à la suite de la formation }\end{array}$ & \\
\hline 17.00 & & CÉRÉMONIE DE CLÔTURE & \\
\hline
\end{tabular}




\section{FORMATION DE RECYCLAGE DES MENTORES}

Cette session d'une heure et demie est destinée à être utilisée pendant les formations de recyclage des mentores pour passer en revue les expériences positives et les défis rencontrés pendant la facilitation et pour discuter des expériences avec l'éducation financière, qui est un élément clé de nombreux programmes pour les AJF. La langue peut être modifiée pour être applicable aux leçons sur d'autres sujets.

\section{Durée : 2 heures}

Thème : Examen du feedback et de l'éducation financière, expériences dans des espaces sûrsObstacles et découvertes positives

\section{Objectifs de la session :}

- Les participantes partagent et discutent des défis et des expériences positives en matière de facilitation de l'éducation financière dans des espaces sûrs.

- Partager les recommandations de l'étude de marché sur amélioration de l'éducation financière

Méthodologie : Discussion de groupe

\section{Matériel :}

- Flip chart

- Marqueurs

- Une image dessinée à la main d'une mentore en voyage, à pied ou à vélo, montrant la distance parcourue ainsi que la route à parcourir. À côté de l'image, il doit y avoir une belle scène de verdure, et de l'autre côté, des entraves éventuelles comme des obstacles, des pierres d'achoppement, etc.

\section{Processus :}

1. Divisez les participantes en groupes de 7 pour constituer 5 groupes (varie en fonction de la taille globale du groupe)

2. Chaque groupe doit choisir un preneur de notes et un présentateur

3. Affichez le dessin sur le mur et attirer l'attention des participantes sur ce point

4. Demandez à chaque groupe de discuter des déclarations et des questions suivantes en se basant sur l'image :

- Il s'agit d'une mentore dans le programme (toute comme vous) qui facilite l'éducation financière (EF) dans des espaces sûrs depuis le lancement du programme
- Dans son parcours de facilitation de l'EF, elle a fait face à des défis, des réalisations, des obstacles et des découvertes positives

- Que diriez-vous de ses connaissances et de sa compréhension de l'éducation financière avant qu'elle ne commence à faciliter l'EF dans les espaces sûrs?

- Quels sont les défis et les obstacles auxquels elle a été confrontée pour faciliter l'EF dans les espaces sûrs et comment a-t-elle fait face?

- Quelles réalisations et quelles expériences positives a-t-elle fait en tant qu'animatrice et mentore dans le domaine de l'EF ?

- Quels sont les sujets qui ont été les plus difficiles à traiter?

- Quels sont les sujets d'EF les plus populaires et plus attrayants pour les filles dans les espaces sûrs?

- Comment les filles ont-elles réagi par rapport à l'argent et à sa gestion dans les espaces sûrs?

- Partagez au moins deux exemples de réussite (par groupe) en termes de l'EF.

- Comment la mentore peut-elle être accompagnée à mieux exécuter l'EF dans les espaces sûrs?

Discussion de groupe : 20 minutes

Présentations de groupe : 50 minutes

Synthèse et conclusion par l'animateur : 20 minutes 


\section{CÉRÉMONIE DE CLÔTURE DE LA FORMATION DES MENTORES}

Cette session de réflexion peut être utilisée pour clore officiellement une formation des mentores.

1. Demandez à tout le monde de se mettre en cercle.

2. Distribuez des bougies à tout le monde.

3. Expliquez que pendant cette formation, nous avons appris à nous connaître et avons appris les unes des autres.

- Chacune de nous a partagé quelque chose de spécial avec le groupe.

- En tant que mentores, vous apprendrez à vous connaître et à apprendre des filles.

- Vous partagerez beaucoup de vousmême, mais vous profitez aussi beaucoup des filles du groupe "espace sûr»

4. Lisez la déclaration suivante : «Une bougie ne perd rien en allumant une autre bougie.»

5. Demandez au groupe la signification de cette affirmation. Discutez.

6. Éteignez les lumières.
7. Résumez le sens de la déclaration tout en allumant votre bougie.

- Quand vous partagez votre lumière, votre passion, votre amitié avec les membres de votre groupe, vous brillerez toujours autant. Vous ne perdrez rien, vous ne ferez que profiter de l'expérience.

- Lorsque vous partagez votre lumière avec des filles, une par une, chaque fille rend le monde plus lumineux.

- Puis tournez-vous et passez la flamme de votre bougie à la personne à côté de vous, puis cette personne tournera et passera la flamme à la prochaine personne, et ainsi de suite dans la salle.

8. Continuez à indiquer comment la pièce devient plus lumineuse par ce partage, mais que personne n'a rien perdu en contribuant avec sa flamme amie.

9. Lorsque toutes les bougies sont allumées, demandez si quelqu'un a quelque chose de plus à dire.

10. Terminez par un discours approprié, puis soufflez toutes les bougies. 


\section{BALIKA : RENFORCER LA CAPACITÉ DES MENTORES AVEC DES SESSIONS DE DÉMONSTRATION HEBDOMADAIRES}

\begin{abstract}
BALIKA (Association bangladaise pour les compétences de vie, le revenu et les connaissances pour les adolescentes) a testé trois approches de renforcement des compétences pour autonomiser les AJF dans le but de retarder le mariage d'environ 9000 filles âgées de 12 à 18 ans dans trois districts du sud-ouest du Bangladesh.
\end{abstract}

L'intervention a permis de fournir une éducation, un soutien, une formation sur les compétences de la vie courante et une formation sur les moyens de subsistance par le biais de groupes hebdomadaires de AJF dirigés par des mentores. Étant donné le rôle des mentores dans l'animation des sessions de groupes de filles, il était essentiel de leur fournir une formation et un soutien continu. Au début du programme, les mentores de BALIKA ont suivi une formation résidentielle complète de quatre jours sur leur rôle de mentore, les compétences de base en matière de vie quotidienne, les techniques d'animation et l'utilisation des ordinateurs et des tablettes. Selon le type d'intervention qu'elles géraient, certaines mentores ont suivi une formation résidentielle supplémentaire de trois jours sur les droits des femmes et la sensibilisation et/ou une formation sur les moyens de subsistance. Au bout d'un an, les mentores ont suivi une formation de recyclage.

Pendant la mise en œuvre du programme, BALIKA a utilisé un outil appelé "sessions de démonstration simulée » où, chaque semaine, 7 à 8 mentores des sousdistricts voisins se réunissaient avec leur superviseur dans les bureaux du programme pour s'entraîner à animer leurs sessions pour la semaine suivante. Ces rencontres en petits groupes ont permis d'améliorer la qualité des sessions et les compétences des mentores en matière d'animation, ainsi que de fournir aux mentores un feedback régulier de la part des pairs et des superviseurs afin de présenter les sujets de BALIKA de manière plus efficace. Les rencontres ont servi à évaluer en permanence les performances des mentores et ont permis à l'équipe du programme d'identifier et d'inviter les responsables à représenter le groupe lors d'événements locaux et nationaux. Les rencontres hebdomadaires ont également donné aux mentores l'espace et le temps de mieux se connaître, de se soutenir mutuellement, d'apprendre les unes des autres comment faire face aux défis de la mise en œuvre sur le terrain et de soulever les problèmes d'une voix collective.

Avec la mise en place de BALIKA, les experts locaux tels que les prestataires de soins de santé et les fournisseurs de services informatiques utilisent des sessions de démonstration hebdomadaires simulées pour dispenser une formation spécialisée dans les domaines de programme pertinents. L'équipe de BALIKA a réalisé que le modèle de réunions hebdomadaires fonctionne bien lorsque les mentores vivent à proximité les unes des autres et du bureau du programme. Si les mentores doivent parcourir de longues distances pour se rencontrer chaque semaine, le modèle peut ne pas être aussi effectif et peut devoir être adapté.

Pour plus d'informations : Retarder le mariage des enfants grâce à des programmes communautaires de développement des compétences pour les filles : résultats d'une étude contrôlée randomisée dans les zones rurales du Bangladesh. 2016. https://knowledgecommons.popcouncil.org/departments_sbsr$\mathrm{pgy} / 557 /$ 


\section{CHAPITRE 3}

\section{Former des groupes communautaires et offrir un contenu principal}

\begin{tabular}{|c|c|c|}
\hline ACTIVITÉ & Exercices d'introduction et brise-glace & Page \\
\hline ACTIVITÉ & Formation de groupes & \\
\hline ACTIVITÉ & Définir des règles de base & \\
\hline GUIDE & Facilitation : astuces pédagogiques pour les mentores & \\
\hline GUIDE & Mobiliser les mentorées et les parents & \\
\hline OUTIL & Planification des sessions & \\
\hline ACTIVITÉ & Jeux et exercices participatifs & \\
\hline ÉTUDE DE CAS & $\begin{array}{l}\text { Initiative pour les adolescentes - Kenya : Adaptation du } \\
\text { programme pour les mentores faiblement d'alphabétisées }\end{array}$ & \\
\hline
\end{tabular}




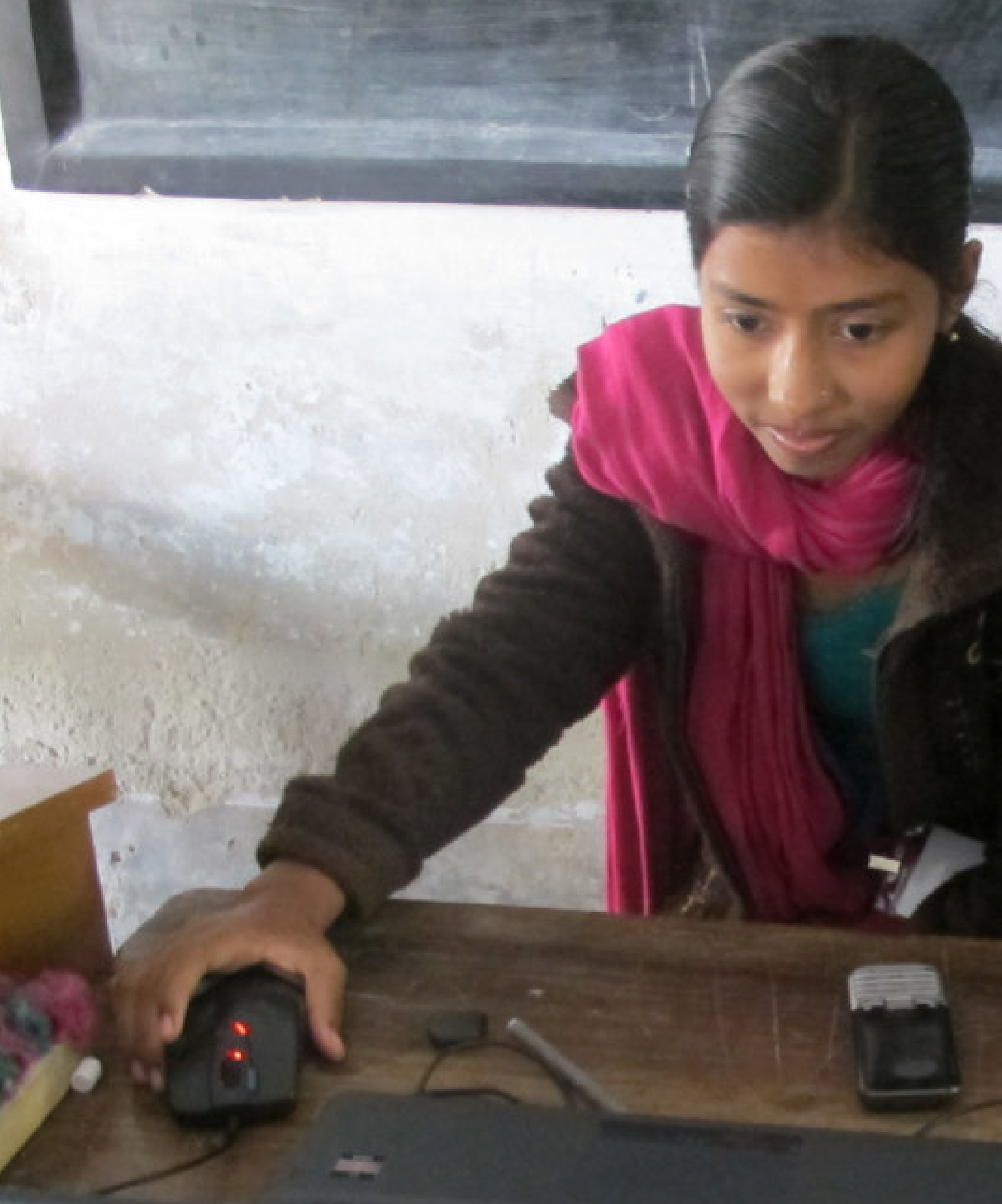




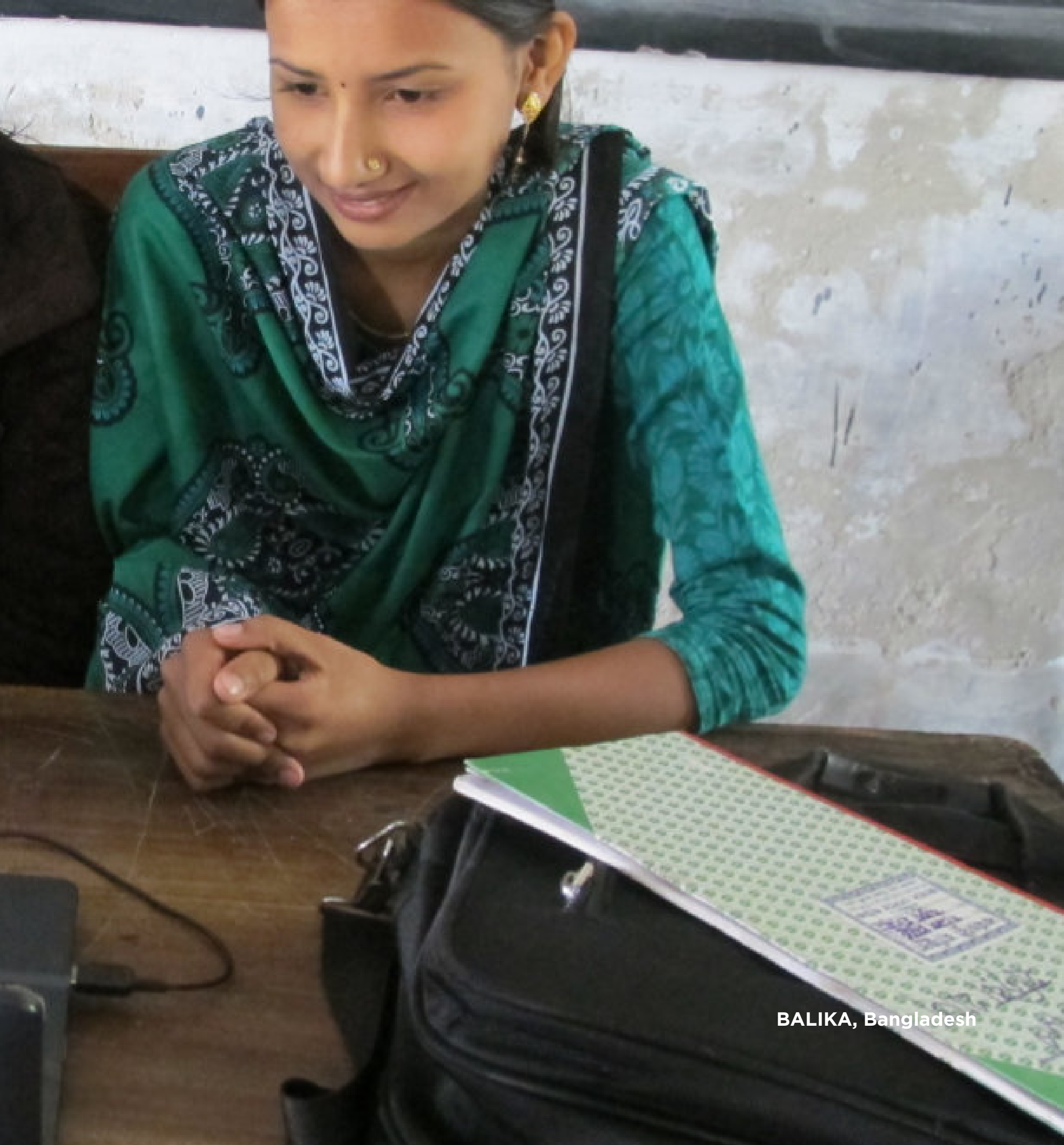


Une fois les mentores formées, leur travail principal commence : Former des groupes de AJF qui se ressemblent (en termes d'âge et de conditions de vie), et dispenser des enseignements pertinents de manière attrayante et interactive.

Dans certains programmes, les mentores sont jumelées pour co-diriger des groupes de 20 à 30 filles qui se rencontrent régulièrement tout au long du programme. Le jumelage des mentores peut faciliter l'animation, diviser les tâches et fournir un soutien au cas où une mentore manquerait une session.

Pour certains exercices du programme, les mentores créent des groupes plus petits pour les discussions et les exercices. Les outils présentés dans cette section comprennent des exemples de moyens amusants par lesquels les mentores peuvent regrouper les filles en sous-groupes plus petits. II y a également des exemples de tâches d'introduction que les mentores feront avec le groupe complet, telles que : établir des règles de base pour les groupes et les brise-glace.

Cette section comprend également des conseils pour introduire les techniques de facilitation participative qui sont utilisées pour fournir le contenu clé des programmes. Les mentores y trouveront des informations sur la manière de parler de questions sensibles, de gérer les émotions et de travailler avec les parents. 


\section{EXERCICES D'INTRODUCTION ET BRISE-GLACE}

\section{Introductions de groupe ${ }^{21}$}

Lors du premier rencontre du groupe, toutes les participantes doivent se présenter. Les mentores peuvent rendre cette présentation aussi amusante ou aussi sérieuse qu'elles le jugent approprié.

Voici quelques idées pour les présentations:

- Les participantes peuvent donner leur nom et leur école, ou la région où elles vivent. Ou les mentores peuvent demander à chaque personne de dire quelque chose de spécial sur elle-même, pour aider les autres à se souvenir d'elle.

- Faites le tour et demandez aux participantes d'indiquer leur nom, leur nourriture préférée ou leur animal préféré. Les mentores peuvent être créatives et penser à une autre catégorie qui peut être plus agréable ou plus pertinente pour leurs participantes.

- Distribuez du papier et des marqueurs ou des crayons de couleur à chaque participante. Demandez aux participantes de faire un dessin qui indique aux autres quelque chose d'important sur elles-mêmes. Donnez cinq minutes pour dessiner. Puis, une par une, demandez à chaque personne de donner son nom et d'expliquer son dessin. De plus, les mentores peuvent rassembler les dessins, les afficher sur le mur ou sur un arbre et, plus tard dans la journée, organiser un concours pour voir qui peut associer les noms aux dessins.

- Demandez aux participantes de former des paires et dites aux partenaires de se présenter les unes aux autres. Donnez-leur cinq minutes pour se parler.

- Demandez ensuite à chaque personne de présenter son partenaire au groupe.

- Demandez aux participantes de former un cercle. Expliquez que chaque personne doit donner certaines informations lorsqu'elle se présente (les mentores peuvent ajouter "quelque chose de différent ou de spécial à votre sujet » aux informations habituelles comme le nom, l'école, la nourriture préférée ou les principaux centres d'intérêt). Ensuite, lancez une balle molle (ou une chaussette roulée) à la personne du cercle qui se présente. Après s'être présentée, elle lance la balle à quelqu'un d'autre dans le cercle. Le jeu se poursuit jusqu'à ce que chaque personne ait eu l'occasion de se présenter.

\section{Les exercices de brise-glace}

Les «brise-glace » et les «Énergiseurs» (activités de réveil) peuvent être utiles tout au long du programme pour aider les filles à se connaître et à garder leur énergie pendant les sessions. ${ }^{22}$ Les «brise-glaces » ou «énergiseurs » sont des activités simples et rapides qui aident les participantes à se détendre, à se sentir plus à l'aise et à (re)connecter avec tout en les incitant à se concentrer et à participer.

Chaque session doit s'ouvrir par une rapide mise en place d'un brise-glace. Essayez d'en choisir une nouvelle à chaque fois et essayez de limiter le temps consacré à cet exercice à environ cinq minutes. Les brise-glace peuvent également être utilisés au milieu d'une session pour "réveiller » les participantes si le niveau d'énergie du groupe semble faible; utilisée de cette façon, l'activité est connue comme un énergisant. Les participantes elles-mêmes ont souvent de bonnes idées d'énergisants et de brise-glace. Donnez aux participantes quelques options ou demandez-leur des suggestions pour un jeu ou une chanson rapide pour démarrer une session. ${ }^{23}$

${ }^{21}$ https://knowledgecommons.popcouncil.org/departments_sbsr-pgy/590/

$22 \mathrm{http}: / /$ aidsalliance.org/resources/467-100-ways-to-energise-groups

${ }^{23}$ https://knowledgecommons.popcouncil.org/departments_sbsr-pgy/584/ 


\section{FORMATION DE GROUPES ${ }^{24}$}

La disposition des participantes en paires ou en petits groupes encourage le travail d'équipe et l'apprentissage participatif et interactif.

\section{Groupes/équipes}

- Comptez. Par exemple, demandez aux participantes de compter par 4. Une par une, chaque participante prononcera un chiffre (la première participante dit « 1 », la seconde dit « 2 », et quand ce sera le tour de la cinquième participante, elle recommencera à 1). Lorsque tout le monde a compté, demandez aux participantes de former des groupes avec des personnes qui ont le même nombre. Au final, vous aurez 4 groupes.

- Créez des puzzles simples de 3 à 5 pièces chacun. Distribuez les pièces du puzzle aux participantes et demandez-leur de trouver toutes les autres qui ont les pièces pour terminer leur puzzle. Assurez-vous d'utiliser des puzzles simples et d'avoir le même nombre de pièces de puzzle que les participantes.

- Demandez aux participantes de faire la queue dans l'ordre de leurs anniversaires - mois et jour seulement - puis comptez (de 1 à 3 par exemple) pour former des groupes.

- Attribuez des couleurs, des symboles ou des images au hasard. Demandez aux participantes de trouver toutes les autres avec la même couleur, le même symbole ou la même image.

- Placez les noms de quatre stars de la culture pop bien connues à différents endroits de la salle et demandez aux participantes de se tenir à côté de leur favori. Si tout le monde rejoint la même star, ajustez l'exercice pour créer plus d'un groupe. Par exemple, demandez aux participantes de passer à leur deuxième star préférée.

- Sélectionnez quatre animaux différents (ou le nombre de groupes que vous souhaitez créer) et écrivez le nom de chaque animal sur plusieurs bouts de papier. Le nombre de feuillets dépendra du nombre de participantes dans chaque groupe. Par exemple, s'il y a 16 participantes, formez quatre groupes de quatre personnes. Dans ce cas, écrivez le nom de chaque animal sur quatre feuilles de papier. Chaque participante tirera un bout de papier d'un récipient. Dites aux participantes que lorsque le mot «maintenant » est prononcé, chaque personne doit faire le bruit de l'animal écrit sur son bout de papier. Tout en faisant leurs bruits, les participantes doivent chercher et écouter ceux qui font le même cri d'animal qu'elles font. Ces personnes sont les membres de leur groupe.

- Que tout le monde croise les bras sur la poitrine. Étonnamment, il y a toujours environ $50 \%$ de personnes qui croisent leurs bras droits sur le gauche, et les $50 \%$ restant le bras gauche sur le bras droit. Demandez aux participantes de fermer les yeux, puis de mettre leurs mains ensemble pour que leurs doigts s'emboîtent et que leurs paumes se touchent. Demandez-leur d'ouvrir les yeux et de regarder leurs mains. Si leur pouce droit est en haut, ils forment une équipe et si leur pouce gauche est en haut, elles vont à l'autre équipe. 
- Formez des paires en divisant le nombre total de participantes par deux. Demandez aux participantes de compter jusqu'au nombre qui représente la moitié du total. Par exemple, s’il y a 20 participantes, elles compteront jusqu'à 10 puis recommenceront à 1. Les deux personnes qui ont le même nombre sont partenaires (les 1, 2, 3, etc.).

- Demandez aux participantes de se tourner vers la personne à leur droite (ou à leur gauche). Cette personne est leur partenaire.

- Créez des ensembles de paires construites autour de mots opposés ou d'objets similaires ou des noms et prénoms de personnes célèbres. Le nombre de paires que vous créez doit être égal à la moitié du nombre de participantes. Chaque paire doit avoir deux parties. Écrivez un mot de chaque paire sur une feuille de papier. Demandez aux participantes de tirer un bout de papier d'un récipient et de trouver l'autre moitié de leur paire. Ceci est leur partenaire. Voici quelques exemples pour un groupe de 10 participantes:

\begin{tabular}{|l|l|l|l|l|l|}
\hline \multicolumn{2}{|l|}{ Opposés } & \multicolumn{2}{l|}{ Objets correspondants } & \multicolumn{2}{l|}{ Des personnes célèbres } \\
\hline noir & blanc & lit & draps & Oumou & Sangare \\
\hline triste & content & table & chaise & Aoua & Keita \\
\hline sombre & clair & caméra & photo & Adam & Bah Konaré \\
\hline grand & petit & voiture & chauffeur & Ellen & Johnson Sirleaf \\
\hline large & étroit & chaussures & chaussettes & Simone & Gbagbo \\
\hline
\end{tabular}

Le thème du groupe peut servir d’inspiration pour la sélection de mots appariés. Ou sélectionnez des personnes célèbres qui correspondent à la tranche d’âge des participantes. Par exemple, les stars de la musique, du sport et du cinéma ont tendance à bien travailler avec les jeunes.

- Demandez à chacune de choisir un nombre entre 1 et (choisissez le nombre supérieur en fonction de la taille de votre groupe). Alignez-les en fonction du numéro qu'elles ont choisi. Les deux personnes en bout de ligne deviennent partenaires, les deux suivants deviennent partenaires, etc. Celles qui ont choisi le même numéro deviennent partenaires. Si une seule personne choisit un numéro particulier, demandez-lui d'en choisir un autre.

- $\quad$ Les groupes s’alignent selon n’importe quelle variable. Les exemples sont : du plus vieux au plus jeune; du plus haut au plus petit; par ordre alphabétique du prénom ou du nom de famille; chronologiquement par mois et date d'anniversaire. Rendez le processus plus difficile en ne permettant pas aux participantes de parler. Les deux personnes au bout de la ligne deviennent partenaires, les deux suivants deviennent partenaires, etc.

\section{La disposition des sièges}

À encourager les participantes à ressentir un sentiment d'appartenance à un groupe et à participer pleinement, il est fortement recommandé de s'asseoir en cercle plutôt qu'en rangées. Cette disposition des sièges permet un contact visuel entre les participantes et l'animatrice crée une atmosphère plus détendue et encourage la participation. 


\section{DÉFINIR DES RÈGLES DE BASE}

Les règles de base des activités sont essentielles pour gérer les sessions de groupe. Les règles de base aident à maintenir la paix et l'ordre, et fournissent un cadre pour que les participantes soient respectueuses et productives. Les règles doivent rester visibles durant toutes les sessions et répétées selon les besoins tout au long des modules. Voici quelques exemples de règles de base :

- Ecoutez ce que les autres - Les opinions et déclarations de disent.

- N'interrompez pas les autres.

- Soyez courtois et apportez votre soutien aux autres.

- $\quad$ Si quelqu'un ne souhaite pas parler, il n'est pas obligé de le faire.

- Ne riez pas de ce que les autres disent.

- Les insultes ne sont pas permises. toutes les filles ont la même valeur.

- Toutes les expériences seront partagées dans un climat de confidentialité et de confiance.

- Si vous souhaitez intervenir, levez la main et attendez qu'on vous donne la parole.

- Les questions sont encouragées et peuvent être posées à tout moment. II n'y a pas de question stupide. Il est normal de rougir, de se sentir gêné ou de ne pas connaître les réponses à toutes les questions.
- L'animatrice peut choisir de ne pas répondre à une question devant les participantes.

- Les choses partagées resteront strictement confidentielles. Elles ne seront pas discutées en dehors du groupe.

- Ne jugez pas les gens à cause de ce qu'ils font ou disent.

\section{Activité}

Les participantes créent une liste de règles de base pour toutes les sessions et font un brainstorming sur une liste de ressources communautaires

\section{Objectifs}

À la fin de cette session, les participantes seront en mesure de:

- Établir, négocier et convenir de règles de base en tant que groupe.

- Disposer d'une liste de ressources communautaires en matière de santé et droits sexuels et reproductifs, de VBG et d'autres services.

\section{Durée}

1 heure (brise-glace, 10 minutes ; révision, 10 minutes; règles de base, 20 minutes; brainstormings sur les ressources et les contacts communautaires, 20 minutes)

\section{Matériel}

1. Flip chart et marqueurs

2. Une liste complète des ressources communautaires locales et des coordonnées des services de santé sexuelle et reproductive; des tests de dépistage du VIH et des conseils; comment et où signaler les cas de violence sexuelle ou domestique ; des conseils psychosociaux ; I'orientation de toute fille vulnérable ayant subi des abus ou un viol; des services juridiques. (II peut être utile de produire des fiches avec des images de ces ressources communautaires.)

Travaux préparatoires d'avant session

- Lisez toute la session et, si nécessaire, entraînezvous à présenter les activités.

- Préparez tout le matériel nécessaire pour la session.

- Discutez avec les membres de la communauté pour vous assurer que le plus grand nombre de personnes et d'endroits possible figurent sur la liste des ressources communautaires et que toutes les coordonnées sont à jour et correctes. 
Termes

- Normes de groupe : Les comportements et caractéristiques standards d'un groupe

- Règles de base : Règles de base définies pour vous aider à vous sentir en sécurité et respecté, et à maintenir votre productivité

- Ressource communautaire : Une organisation, une institution, un groupe ou une personne de la communauté qui fournit une assistance et un soutien aux personnes à risque

\section{Brise-glace}

- Ouvrez la session avec un brise-glace de votre choix ou permettez aux participantes d'en suggérer un.

- Il est opportun de choisir une activité briseglace ou de consolidation de la confiance plus longue en raison du temps relativement court nécessaire pour couvrir le contenu de cette session.

\section{Procédure}

Demandez:

Connaissez-vous les règles de base ou les normes de groupe ? Pourquoi pensez-vous qu'il est important d'établir des règles de base? (Comprendre les limites et les libertés d'un contexte, améliorer le travail en équipe, assurer la productivité et le respect)

Quelles sont des situations réelles dans lesquelles il est important d'avoir des règles ? (Par exemple, quand quelqu'un vole quelque chose, quand il y a un rencontre et tout le monde parle en même temps, quand quelqu'un parle et que d'autres font des commentaires négatifs à propos de sa contribution à la conversation, quand tout le monde contribue aux tâches ménagères, etc.)

\section{Expliquez aux participantes:}

Des règles de base ou des normes de groupe sont établies pour vous aider à vous sentir en sécurité en participant à ces réunions. Bien qu'il s'agisse d'un programme participatif, vous ne serez pas obligé de participer plus que vous ne le souhaitez.
Sachez qu'il s'agit d'un environnement sûr dans lequel vous pouvez partager vos pensées et vos sentiments, et que le partage de vos propres questions ou histoires peut aider d'autres filles qui peuvent avoir vécu ou s'interroger sur des choses similaires. Les règles de base aident à garantir que chacune aura la chance d'être entendue sans jugement ni ridicule, et que ses limites personnelles et sa vie privée seront également respectées.

\section{Demandez:}

Quelles sont les règles de base que vous souhaitez définir pour ces réunions? (Par exemple : ce qui se dit ici, doit rester ici ; chacune est libre de participer ou pas; ne pas rabaisser les autre pour leurs sentiments, leurs opinions ou leurs expériences ; être respectueuses les unes envers les autres, s'écouter et ne pas interrompre, etc.)

Notez les idées de règles de base sur un flip chart et affichez-les pour référence tout au long du programme. (Écrivez en termes simples [en utilisant également des images] pour s'adapter à tous les niveaux d'alphabétisation.)

Demandez maintenant : Quelles punitions interactives souhaiteriez-vous imposer à celles qui enfreignent les règles de base ? (Par exemple, le contrevenant doit faire 10 sauts de grenouille, ou écrire au tableau ou sur le flip chart [ou dire à haute voix trois choses qu'elle a apprises jusqu'à présent], le contrevenant doit chanter un couplet d'une chanson.)

Notez également ces réponses sur un flip chart et affichez-les pour référence tout au long du programme. Expliquez ensuite aux participantes qu'elles voteront maintenant pour s'entendre sur ces règles en levant la main. Expliquez que ce vote servira d'accord, ce qui signifie que le groupe a accepté de suivre ces règles à chaque rencontre du groupe de filles. Lancez le vote. Si une ou plusieurs filles ne lèvent pas la main, demandez pourquoi et discutez de ce qui devrait être modifié dans les règles pour que tout le monde les accepte. 


\section{FACILITATION : ASTUCES PÉDAGOGIQUES POUR LES MENTORES}

Pour la plupart des mentores et des participantes au programme, la facilitation plutôt que de se contenter de faire un cours magistral, est une nouvelle façon d'interagir. Les astuces suivantes donnent des suggestions pour créer un environnement d'apprentissage positif, traiter des questions sensibles et pour bien se préparer avant une session. ${ }^{25}$

\section{Créer un environnement d'apprentissage approprié}

N'oubliez pas que la mentore n'a pas toutes les réponses. Les participantes viennent aux sessions d'apprentissage avec une grande expérience et ont beaucoup de choses à apporter. Lors de la session, il est important qu'aussi bien les mentores que les participantes enseignent et apprennent les unes des autres.

- Créez un environnement d'apprentissage sûr.

- Faites un feedback aux participantes et félicitezles pour leurs efforts.

- Réfléchissez aux moyens de rendre le sujet accessible à tous les participantes présentes.

- Faites comprendre aux participantes que vous êtes aussi un apprenant au même titre qu'elles, même si vous êtes un leader et un mentor.

- Promouvez des conversations et des questions qui permettent aux jeunes d'explorer et de clarifier leurs valeurs en même temps qu'elles développent un sentiment de soi.

- Utilisez une variété d'activités adaptées aux différents niveaux de maturité pour couvrir les nombreux stades de développement des jeunes.

- Rendez l'apprentissage pertinent à la situation des jeunes. Soyez prudents lorsque vous leur demandez de se projeter loin dans l'avenir.

- Anticipez que les jeunes peuvent avoir des expériences limitées sur un sujet. Les histoires peuvent servir à remplacer l'expérience de la vie réelle.

- Assurez-vous que tout au long de la session, vous leur donnez une possibilité de penser, d'agir et de sentir.

- Incluez les médias actuels pour garantir la pertinence du sujet et encourager la discussion.

- Engagez les familles et les communautés des participantes à s'intéresser au programme, à comprendre son contenu et à aider les jeunes à changer de comportement et à atteindre leurs objectifs.

- Encouragez l'humour et utilisez des jeux interactifs afin que la formation soit dynamique et amusante!
Créer un environnement d'apprentissage basé sur l'égalité, le respect et les droits humains

Créez un environnement propice à l'apprentissage. Toutes les participantes doivent se sentir impliquées, écoutées, confortables et à l'abri du ridicule, surtout lorsqu'elles prennent le risque d'exprimer de nouvelles idées.

- Encouragez la participation, en particulier de celles qui se sentent seules ou intimidées. (Diverses conditions et situations peuvent déclencher de tels sentiments. Par exemple, les différences de pouvoir social liées au genre, à la classe sociale ou à l'âge. Les participantes ayant une maîtrise limitée de la langue parlée à l'école sont susceptibles d'éviter de se joindre aux discussions. Les personnes vivant avec un handicap physique ou d'autres besoins particuliers peuvent être réticentes.) Boostez la participation du groupe en utilisant des activités qui encouragent le respect et l'esprit d'équipe et en favorisant des possibilités de leadership. II est également essentiel de garantir la sécurité et la confidentialité de l'espace physique.

- Au cours d'une des premières sessions, demandez aux participantes de travailler ensemble pour élaborer une liste de lignes directrices afin de garantir le respect au sein du groupe.

- Demandez aux participantes de respecter la vie privée des autres, et rappelez-leur de ne pas divulguer des informations à d'autres personnes qui, à leur avis, devraient rester confidentielles. Réfléchissez à la manière dont certaines participantes pourraient intimider ou même abuser d'autres personnes après avoir terminé une session de groupe au cours de laquelle des sujets sensibles ont été abordés. Assurez-les que la mentore gardera toutes les discussions confidentielles. De cette manière, les mentores peuvent servir de modèle pour le respect de la vie privée des autres.

- Assurez-vous qu'elles comprennent qu'elles ont le droit de ne pas participer ou de ne pas partager si cela les met mal à l'aise. 


\section{Conseils généraux sur l'animation de groupes}

- Favorisez un processus qui s'appuie sur les expériences des participantes, en intégrant de nouvelles informations et idées à ce qu'elles savent et pensent déjà sur le sujet.

- N'oubliez pas que toutes les participantes ont des connaissances et des expériences propres. Écoutez-les et posez des questions pour les amener à participer.

- Écoutez leurs préoccupations et posez des questions hypothétiques sur des problèmes qui correspondent à leur vie réelle. Encouragez-les à résoudre ces problèmes, en faisant leur propre choix et en développant leurs idées en même temps.

- Encouragez-les à explorer la signification d'un problème dans leur propre vie.

- Considérez le rôle de la mentore comme étant celui d'un facilitateur de la discussion des idées des participantes plutôt que celui d'un professeur ou d'un relais d'information. Parfois, une mentore peut avoir besoin de corriger des erreurs factuelles ou d'aider les participantes à reconnaître quand un commentaire est irrespectueux.

- Il peut parfois être utile de partager un exemple tiré d'une expérience personnelle qui est pertinent pour la leçon. Cependant, soyez extrêmement attentive à garder des limites appropriées avec les participantes.

Développer le potentiel de réflexion et d'analyse critique dont disposent les participantes sur leur propre vie et sur le monde qui les entoure, ainsi que leur capacité à résoudre les problèmes

- Encouragez les participantes à remettre en question les idées reçues. Demandez-leur de réfléchir à leurs croyances et aux normes de leur communauté. Invitez-les à examiner des opinions différentes des leurs d'une manière respectueuse.

- Encouragez la créativité.

- Encouragez les participantes à prendre des risques dans leur réflexion et à ne pas avoir peur de faire des erreurs. Soyez prête à démontrer vous-même un tel comportement.

- Organisez de petits groupes pour explorer certains enjeux et pour participer à des activités collectives.

- Encouragez différentes perspectives dans l'analyse des problèmes et dans la recherche de solutions possibles.

\section{Un bonne facilitatrice}

- Considère les participantes comme des experts en matière d'information et de compétences à partager, plutôt que de se voir comme le seul expert présent dans la salle.

- Encourage les participantes à apprendre les unes des autres et guide ce processus plutôt que de présenter des instructions et de dispenser des cours magistraux.

- Considère que nous apprenons en faisant, en expérimentant, en pratiquant et en ressentant, plutôt qu'en mémorisant, en répétant et en enregistrant les informations.

- Est organisée, mais est flexible pour adapter ses méthodes en fonction des besoins des participantes.

- Est motivée par le sujet et les participantes.

- Tient ses promesses envers le groupe (de laisser les participantes parler, faire une pause, etc.)

- Est patiente et sait écouter.

- Est capable de faire face à des émotions fortes qui pourraient surgir au cours des discussions.

- Encourage la capacité des participantes à appliquer ce qu'elles apprennent à leur vie et à leur communauté, c'est-à-dire qu'il les aide à devenir des citoyennes actives et des forces de pour un changement positif.

- Demande à chaque fois aux participantes de faire le lien entre le contenu et leur propre vie et leur monde. Elle les encourage à considérer les informations à la lumière des principes d'équité et de justice sociale.

- Est consciente de l'environnement dans lequel elle travaille afin d'éviter de mettre les participantes en danger.

- Garde à l'esprit que les jeunes s'inspirent souvent de leurs enseignantes et des dirigeantes de leur communauté. 


\section{FACILITATION: CONSEILS PÉDAGOGIQUES POUR LES MENTORES (suite)}

\section{Préparation des sessions}

\section{Avant chaque session:}

- Lisez attentivement tous les documents/supports relatifs à la session et aux activités. Réfléchissez à la manière dont vous réaliserez chaque étape et ce que vous ajouterez à chaque session.

- Essayez d'anticiper les questions qui pourraient être posées, et sachez où chercher des réponses dans les documents fournis. Si les participantes posent des questions auxquelles vous ne pouvez pas répondre tout de suite, notez-les et recherchez les réponses avant la prochaine session, afin que vous puissiez en discuter en ce moment.

- Adaptez les activités et les discussions de groupe pour les rendre plus appropriées à l'âge et au niveau d'éducation de vos participantes.

- Réfléchissez et prévoyez les problèmes qui pourraient surgir lors de sessions plus "difficiles» avec un exercice complexe ou couvrant des sujets sensibles.

- Pensez à des exemples locaux et à des moyens de rendre les activités plus pertinentes par rapport à la vie quotidienne et aux préoccupations des participantes. Si possible, abordez d'abord des sujets faciles et puis des sujets plus difficiles.

- Préparez les supports/la documentation à I'avance.

\section{Quelques conseils de facilitation supplémentaires :}

- Disposez d'un « coin de stockage » pour les questions qui se posent lors d'une session, mais qui ne sont pas pertinentes quant aux objectifs de la session. Vous pouvez y revenir à un autre moment ou tout simplement expliquer à la fin de la formation que c'était une question importante mais pas en lien avec le sujet de la session.

- Notez les points importants sur un tableau lors des discussions pour y revenir lors de la synthèse des activités. II peut être très difficile de mener à la fois la discussion et de noter tous les points importants, pensez donc de faire appel à quelqu'un pour vous aider à noter les points clés.

- Si possible, définissez les prochaines étapes ou les solutions possibles aux problèmes.

- Quand possible, reliez les informations à ce qui a été appris lors des sessions précédentes.

- Assurez-vous que vous ne parlez pas plus que les participantes. Si vous constatez que vous menez la plus grande partie de la conversation, encouragez les participantes à répondre aux questions des unes et des autres. Par exemple, si quelqu'un pose une question, ouvrez la discussion aux participantes en demandant par exemple : "Est-ce que quelqu'un a une réponse à cette question »?

- Votre langage corporel contribuera à animer efficacement le groupe. Par exemple, le fait de regarder attentivement et de hocher la tête montre aux participantes que leur contribution est importante. Le fait de détourner le regard peut indiquer clairement à la personne qu'elle ne devrait pas parler ou participer. Faites toujours face à la personne à qui vous parlez.

- Amusez-vous! 26 


\section{MOBILISER LES MENTORÉES ET LES PARENTS}

Les programmes utilisent des méthodes d'enseignement participatives, telles que la discussion de groupe, le brainstorming, le jeu de rôle, le travail en petits groupes, des jeux éducatifs et des contes. Les mentores peuvent préférer faire une présentation et peuvent considérer l'animation d'un jeu ou d'une autre exercice interactif un peu compliqué. Cependant, les jeux et les activités offrent aux participantes la possibilité d'interagir librement, et ils génèrent souvent des discussions qui ne peuvent être engendrées par d'autres méthodes. Les techniques participatives offrent également aux participantes la possibilité de s'exercer à utiliser de nouvelles compétences (par exemple, la communication et la prise de décision). N'oubliez pas que les recontresde groupe sont censés être un environnement amusant et détendu, et NON un environnement qui ressemble à une salle de classe ! Imaginez une séance de formation active, parfois bruyante et amusante.

Les avantages des méthodes participatives actives

- Amélioration de la capacité de réflexion critique

- Probabilité accrue que les participantes se souviennent et partagent de nouvelles informations

- Motivation accrue

- Amélioration des compétences interpersonnelles

\section{Exemples de méthodes participatives}

Le brainstorming. Le brainstorming est une exploration d'idées et est un excellent moyen de lancer un sujet de discussion. Lors d'un brainstorming, personne ne doit juger une réponse donnée par quelqu'un. Chaque réponse est simplement notée sur un papier ou un tableau. Cette activité encourage les participantes à étendre leur réflexion à propos d'une idée et à envisager un sujet sous différents angles et perspectives.

La discussion de groupe. La discussion de groupe permet de recueillir les réponses des participantes sur un sujet particulier et donne à l'animatrice la possibilité d'accroítre les connaissances des participantes ou de corriger les informations erronées. L'efficacité de la discussion de groupe dépend souvent de la capacité de l'animatrice à utiliser des questions ouvertes, c'est-à-dire des questions posées par l'animatrice qui nécessitent plus qu'une simple réponse par «oui » ou «non ». Ces questions aident à faire ressortir des sentiments ou des pensées sur un sujet ou une activité.
- «Qu'avez-vous appris de cette activité ? » est une question ouverte. "Dites-moi comment cette activité vous a affecté » est un autre exemple de question ouverte. Les questions ouvertes commencent souvent par «Quoi, quand, pourquoi ou comment ».

- «Avez-vous appris quelque chose ? " n'est pas une question ouverte, car la participante peut simplement répondre par oui ou par non

Jeu de rôle. Le jeu de rôle permet aux participantes de vivre une situation réelle, sans avoir à prendre de risques réels. Il est important que les mentores encouragent les participantes à jouer des situations réalistes, et non des situations idéales. Les règles du jeu de rôle sont les suivantes:

- Discutez de la situation au sein de votre propre petit groupe de jeu de rôle.

- Convenez d'un scénario.

- Convenez de qui fait quoi, en impliquant tout le monde.

- Répétez.

- Jouez pour le groupe entier. ${ }^{27}$

Les histoires. Les histoires peuvent aider à illustrer les principaux thèmes de la leçon du jour. Veillez à lire ces histoires avant de les présenter, afin de les présenter de manière vivante, sans les lire directement depuis la page. Si vous disposez d'une photocopieuse, faites des copies pour chaque fille. 


\section{MOBILISER LES MENTOREÉS ET LES PARENTS (suite)}

Les images: Une autre façon de rendre les sessions plus vivantes et intéressantes serait d'utiliser des images. On peut aussi utiliser des objets réels pour représenter les images.

\section{Intervenants externes}

Les mentores doivent faire appel à un conférencier invité chaque fois que cela leur convient et qu'il est possible de le faire. Les filles auront toujours intérêt à rencontrer et à apprendre des femmes avec lesquelles elles s'identifient et qui peuvent leur servir d'exemples positifs et/ou de modèles. ${ }^{28}$

\section{Travailler avec les parents ou les tuteurs}

- Tenez les parents informés de ce que vous faites et pourquoi. Toutefois, les parents ne doivent pas savoir chaque détail de ce qui est discuté dans le programme, et ce que les participantes partagent lors des sessions de groupe doivent rester confidentiel.

- Obtenez l'avis des parents.

- Parlez aux parents de leurs préoccupations et de leurs craintes autour de la santé reproductive et du VIH et du SIDA (ou d'autres sujets sensibles en rapport avec votre programme, comme le mariage des enfants, les mutilations génitales féminines et la violence basée sur le genre). Pour ce faire, il faut recourir aux recontres avec les parents, les visites à domicile, les rencontres communautaires, etc.

- Invitez les parents à des recontres pour discuter de leurs préoccupations.

- Apprenez à connaître la communauté. Renseignez-vous sur leurs besoins ainsi que sur leurs compétences et l'expertise des parents. Un parent peut être une infirmière dans une clinique locale par exemple, et pourrait parler aux participantes, vous donner des conseils et vous soutenir.

- Proposez des discussions sur les compétences parentales. Faites venir des experts pour présenter leurs idées, aider à améliorer la communication entre parents et enfants et partager des ressources et du matériel.

- Impliquez les parents. Demandez aux parents de contrôler les devoirs et assignez des exercices qui demandent aux participantes de parler à leurs parents. Par exemple, demandez aux participantes d'interroger leurs parents ou les membres de leur famille sur leur jeunesse. ${ }^{29}$

\footnotetext{
${ }^{28}$ https://knowledgecommons.popcouncil.org/departments_sbsr-pgy/588/
}

${ }^{29}$ https://knowledgecommons.popcouncil.org/departments_sbsr-pgy/584/ 


\section{PLANIFICATION DES SESSIONS}

Au cours des ateliers de formation et des recontres réguliers, les mentores doivent étudier les guides du programme d'études et planifier les sessions à venir. La stratégie d'étude et de planification des sessions décrit huit étapes à préparer avant de commencer une session. Idéalement, les mentores se préparent en groupe afin de pouvoir discuter des adaptations des activités proposées dans le guide du programme d'études. Au fil du temps, chaque groupe peut déterminer et concevoir une stratégie de préparation qui lui convient

Lors de la préparation et avant la tenue d'une session, les mentores doivent:

- Connaître et comprendre la question qui sera abordée au cours de la session.

- Comprendre les objectifs fixés pour la session.

- Adapter les activités pour qu'elles soient attrayantes et significatives pour les participantes.

1. Familiarité. Lisez tout le contenu de la session en silence et marquez les mots inconnus ou le contenu qui n'est pas clair.

2. Compréhension du vocabulaire. Écrivez les mots inconnus sur un tableau sous forme d'une liste unique. Les mentores partageront entre elles leurs intuitions sur chaque mot afin de trouver une définition. À la fin, elles peuvent comparer chaque définition avec celle d'un dictionnaire.

3. Objectifs de la session. Lisez les objectifs établis pour la session et discutez de la manière dont chacune d'entre elles renforcera les adolescentes dans la communauté.

4. Questions et concepts clés. Chaque mentore prendra un concept clé et le traduira dans sa langue maternelle (si pertinent), de sorte que le concept soit proposé dans la langue principale du pays et dans la langue locale, si nécessaire. Les mentores ne sont pas censées traduire littéralement les concepts, mais exprimer le sens et la signification du concept clé dans la langue dans laquelle elles travailleront avec les participantes, s'il s'agit d'une langue autre que celle dans laquelle le programme est rédigé.

5. Activités contextualisées. En groupe, les mentores liront la session dans le guide du programme, en énumérant chaque activité. Elles peuvent adapter/modifier/ajouter des activités pour s'assurer qu'elles sont en rapport avec la vie des adolescentes et la culture locale.

6. Programme. Créez un format de programmation pour aider à décrire les activités.

7. Contrôle. Les mentores reviennent sur les objectifs de la session et vérifient qu'ils sont atteints.

8. Matériel et ressources. Préparez le matériel nécessaire pour la session. ${ }^{30}$

\begin{tabular}{|l|l|l|}
\hline Type de session & Nom de la session & Date de la session \\
\hline & & \\
\hline
\end{tabular}

\begin{tabular}{|l|l|l|l|}
\hline Activité & Description de l'activité & Matériel & Durée estimée \\
\hline & & & \\
\hline & & & \\
\hline & & & \\
\hline & & & \\
\hline
\end{tabular}

Source: Abriendo Oportunidades

30 https://knowledgecommons.popcouncil.org/departments_sbsr-pgy/593/ 


\section{JEUX ET EXERCICES PARTICIPATIFS ${ }^{31}$}

\author{
Jeux destinés à faciliter l'éducation financière
}

\section{LA GRANDE FÈVE}

Matériel :

2 jetons

30 fèves/grains de maïs/cailloux

2 verres utilisés comme verres d'épargne pour

déposer les fèves

Objectif : Accumuler le plus grand nombre de fèves dans les verres d'épargne.

\section{Instructions :}

- Deux équipes avec un nombre égal de participantes se forment et s'alignant en rangs.

- Les verres d'épargne sont placés à une extrémité de chaque rangée.

- La première participante de chaque rangée reçoit un jeton, qu'elle doit mettre sous ses vêtements (col de la robe) et retirer au niveau de ses pieds (pantalon, jupe, short). Elle le passe à la suivante pour faire de même et ainsi de suite jusqu'à ce que le jeton arrive à la dernière participante.

- La dernière participante doit se rendre à un point éloigné, où se trouvent les fèves. Elle prend une fève et court jusqu'au début de la rangée pour le déposer dans le verre d'épargne. Le processus est répété plusieurs fois en donnant un nouveau jeton à la première personne de la file pour qu'elle répète l'exercice jusqu'à ce que le temps soit écoulé.

- L'équipe gagnante est celle qui a recueilli le plus de fèves dans leur verre d'épargne.

\section{Réflexion :}

Si nous sommes organisés et que nous avons un plan, nous pouvons faire de meilleures économies.

\section{LA TIRELIRE}

(Une adaptation du jeu Rabbits \& Hutches/Les lapins dans leur terrier)

Matériel : Un grand espace pour jouer

Objectif : Comprendre que l'épargne est une ressource à mettre de côté et à conserver en lieu sûr.

\section{Instructions :}

- Formez des groupes de trois participantes.

- Dans chaque groupe, deux participantes se prennent la main (représentant la tirelire) et une participante est placée entre elles (représentant la pièce de monnaie).

- L'animatrice appelle alternativement « pièce » ou «tirelire ». Quand elle dit « pièce », les participantes quittent la tirelire et cherchent une autre tirelire pour les contenir.

- Quand elle dit «tirelire », les paires se déplacent en cherchant une autre pièce à épargner.

\section{Réflexion :}

- Il est important d'avoir un endroit sûr pour faire des économies.

- Il peut s'agir d'une tirelire, d'une banque, de groupes organisés dans la communauté, ou d'une coopérative. 


\section{LE PASSAGE DANGEREUX DES PIĖCES DE MONNAIE} À L'ÉPARGNE

Matériel :

Un tableau pour chaque groupe

Marqueurs

Une pièce de monnaie

Un verre avec une inscription : Épargne

Objectif : Encourager la communication et l'organisation au sein de l'équipe pour atteindre les objectifs fixés.

\section{Instructions :}

- Formez deux rangées de cina personnes.

- Chaque groupe doit identifier cinq dépenses communes auxquelles les gens font face et qui mettent l'épargne en danger.

- Faites une affiche pour chaque dépense et placezla dans un lieu visible.

- Les groupes forment deux rangées. Le premier membre de chaque rangée reçoit une pièce de monnaie, et un verre portant l'inscription «ÉPARGNE » est placé à l'extrémité opposée de la rangée. Le but est de faire passer la pièce entre elles, en utilisant uniquement leurs pieds, jusqu'à ce qu'elle atteigne le verre à la fin de la rangée. Si la pièce tombe par terre au cours de son trajet, elle sera ramenée au début de la rangée et il faudra recommencer.

- Vous pouvez utiliser autant de pièces que vous le souhaitez pour animer le jeu. Le premier groupe à placer toutes les pièces dans le verre gagne.

\section{Réflexion :}

- Comment les filles ont-elles ressenti le fait de passer la pièce avec leurs pieds?

- Que fallait-il pour réussir à passer la pièce?

- À quelles fins utiliseraient-elles les pièces qu'elles ont collectées?

- Est-il facile ou difficile d'économiser?

- Quels sont les défis que vous rencontrerez en matière d'épargne?

- Comment les femmes peuvent-elles s'organiser pour se soutenir mutuellement dans l'épargne?

\section{CACHEZ LA PIĖCE DE MONNAIE}

Matériel : Plusieurs pièces de monnaie

Objectif : Reconnaître que les économies doivent être mises de côté et ne pas être considérées comme une ressource à dépenser pour des articles inutiles.

\section{Instructions :}

- Les filles sont regroupées par deux et l'une des deux reçoit une pièce de monnaie.

- La fille qui a la pièce la cache dans une de ses mains. Pendant ce temps, le groupe chante une chanson : "Cachez la pièce, cachez la pièce, cachez la pièce, cachez-la bien, cachez-la dans la tirelire pour ne pas la dépenser, celui qui la dépense n'aura pas d'économies. »

- À la fin de la chanson, la partenaire de la fille doit deviner dans quelle main la fille a mis la pièce de monnaie. Si elle devine correctement, c'est à son tour de cacher la pièce, sinon elle continuera à deviner jusqu'à ce qu'elle réussisse.

\section{Variante :}

Toutes les filles sont placées dans un cercle avec leurs mains fermées. La pièce est placée dans la main d'une des participantes, qui doivent la passer de main en main, mais sans ouvrir entièrement leurs mains, en s'assurant que personne ne voit jamais la pièce. Le jeu se termine lorsque la pièce de monnaie a été remise à toutes les participantes.

\section{Réflexion :}

- Pourquoi est-il important que la pièce soit bien conservée?

- Que se passerait-il si nous la voyions tout le temps ou si nous ne l'avions pas dans un endroit sûr?

- Quelles stratégies en matière d'épargne allezvous commencer à mettre en pratique? 


\section{JEUX ET EXERCICES PARTICIPATIFS (suite)}

Jeux destinés à faciliter l'éducation financière

\section{LE FEU DE CIRCULATION}

Objectif : Évaluer le degré de communication qu'existe parmi le groupe de participantes.

\section{Instructions :}

- Une fille mènera le jeu tandis que les autres formeront des paires. Elle dirigera le jeu en chantant «Vert », « Jaune » ou «Rouge » et les paires devront suivre ses instructions :

-Quand elle dit «VERT », les paires se tournent face à face.

-Quand elle dit «JAUNE », les paires se mettent dos à dos.

-Quand elle dit «ROUGE », tout le monde change de partenaire et celle qui reste seule cherche un nouveau partenaire.

- Celle qui reste seule supervise le jeu.

\section{Réflexion :}

- Selon les différentes couleurs, qu'est-ce que la communication entre les personnes ? Le vert indique une bonne communication, le jaune une communication à améliorer et le rouge une communication rompue.

- Comment se passe la communication entre nous?

- Comment résolvons-nous nos conflits?

- Qu'est-ce qui nous unit en tant que groupe?

- Savons-nous comment demander de l'aide lorsque nous en avons besoin? Vers qui nous tournonsnous?

- Que puis-je apporter au groupe pour améliorer nos relations?

\section{LA CHANSON DE LA TIRELIRE}

Objectif : Réfléchir aux bons usages de l'épargne et motiver les participantes à continuer à épargner.

« J'ai une tirelire, une tirelire pour l'épargne; quand j'ai de l'argent, j'y mets un sou.

C'est ce que ma main me fait faire. Ma main me fait faire cela.

J'ai une tirelire, une tirelire pour l'épargne; quand j'ai de l'argent, j'y mets de plus en plus.

Quand elle est bien remplie, je dois acheter: des fruits et des légumes pour manger sainement ».

Donc, petit à petit, je veux épargner Pour que dans mon avenir, je ne cesse jamais d'apprendre. »

\section{LA PIÈCE}

Objectif : Reconnaître que l'épargne exige des efforts, mais que c'est possible.

\section{Instructions :}

- Les participantes forment un cercle en se tenant par la main. On demande à un volontaire d'être la pièce de monnaie et de rester en dehors du cercle; son but est de pouvoir entrer dans le cercle quand elle est appelée à la tirelire.

- Les membres du cercle chantent : «Pièce, pièce, entre dans ma tirelire»

- La participante qui se trouve en dehors du cercle pose la question : où?

- Les membres du cercle ouvrent un espace qui se referme rapidement en disant : par ici.

- Cela sera répété plusieurs fois jusqu'à ce que la fille puisse entrer dans le cercle.

- Et le jeu recommence avec de nouveaux volontaires qui sont prêts à jouer le rôle de pièces de monnaie. Il peut y avoir une seule pièce ou plusieurs à la fois.

\section{Réflexion :}

- Qu'ont ressenti les personnes qui étaient la pièce de monnaie?

- Quel est le rapport avec notre vie en ce qui concerne nos propres épargnes? 


\section{CONCENTRÉ DE TOMATES ET SUPER TOMATE}

Objectif : Comprendre la différence entre les produits de première nécessité et les produits de luxe.

\section{Instructions :}

- Formez deux groupes concurrents.

- Chaque groupe reçoit une feuille sur laquelle figure, d'un côté, le mot «Super Tomate » et de l'autre côté, le mot «Concentré de tomates».

- Du côté «Super Tomate », elles écrivent des choses qui sont des envies, des caprices et des luxes. De l'autre côté, elles écrivent des choses qui sont des nécessités. Chaque groupe fait la liste la plus longue possible en fonction du temps imparti.

\section{Réflexion :}

- Comparez les deux listes et analysez les cas où il n'est pas clair si l'article est une nécessité ou un luxe.

- Estimez le coût des articles de luxe et comparez-le avec le coût des produits de première nécessité.

- Prioriser les dépenses en fonction du degré de besoin/utilité des éléments énumérés.

\section{MOTS CROISÉS SUR L’ÉPARGNE}

Objectif : Identifier les principaux éléments de l'épargne.

\section{Instructions :}

- Installez un flip chart avec les mots croisés incomplets et les lettres qui complètent les mots.

- Les participantes sont invitées à résoudre les mots croisés en remplissant les espaces vides avec les lettres dont elles disposent.

\section{Réflexion :}

- $\quad$ En complétant les mots croisés, les participantes doivent faire des phrases en utilisant les mots qu'elles ont formés.

- Les participantes peuvent également écrire les questions qui servent d'indice pour chacun des mots qu'elles ont placés.
Horizontal
Vertical
1. Pièce de monnaie 1. Épargne
2. Épargner 2. Gagner
3. Tirelire 


\title{
JEUX ET EXERCICES PARTICIPATIFS (suite)
}

\author{
Jeux pour promouvoir l'éducation à la santé sexuelle et reproductive
}

Les jeux et les exercices participatifs soutiennent et renforcent également les leçons tirées des cours de santé sexuelle et reproductive. 32

\section{MOTS CACHÉS}

Instructions : Donnez à chaque équipe une affiche de mots cachés contenant les mots suivants :

Vagin

Utérus

Ovaire

Vulve

Anus

Pénis

Femme

Menstruations

- Les mots cachés sur chaque affiche doivent comporter des mots cachés de manière différente.

- $\quad$ Chaque équipe doit trouver les mots cachés. Si un groupe termine en premier, les filles peuvent aider l'autre groupe à trouver les mots. Elles peuvent ensuite revoir l'emplacement, les fonctions et l'hygiène personnelle pour chacun des mots.

\section{LA CHANSON DU « JE VEUX... »}

Je veux chanter,

Je veux voir,

Je veux ressentir

Je veux toucher,

Et connaître mon corps

Ici, je touche (elles pointent une partie de leur corps)

Jouez plusieurs fois jusqu'à ce que le plus grand nombre possible de parties du corps soit cité.

\section{Réflexion :}

Les filles peuvent réfléchir à l'importance de connaître les différentes parties de leur corps, à quoi ça ressemble et prendre conscience de tout ce qui se passe dans leur corps au fur et à mesure qu'elles grandissent.

\section{SPERMATOZOİDES ET OVULES}

(Une adaptation du jeu Rabbits \& Hutches/Les lapins dans leur terrier)

\section{Instructions :}

- Formez des groupes de trois participantes.

- Deux filles dans chaque groupe se tiendront la main et représenteront l'ovule, et la troisième se trouve au milieu représentant le spermatozoïde.

- Il doit y avoir une ou deux filles sans groupe dont le but est de trouver un groupe auquel ils peuvent se joindre.

- Lorsque la mentore appelle « ovule », celles qui se tiennent la main se déplacent pour chercher une fille représentant le spermatozoïde.

- À ce moment, les filles qui n'avaient pas de groupe au début sont intégrées au jeu, laissant d'autres à l'extérieur.

- La mentore dira alors «spermatozoïde » et seules les filles qui représentent les spermatozoïdes vont se déplacer pour trouver un autre ovule pour les recevoir.

- Les filles qui sont laissées hors-jeu peuvent poser à leurs camarades des questions sur ce qu'elles savent à propos des ovules, des ovaires, du sperme, de la fécondation, de la grossesse, etc.

\section{TUNNEL DE JAMBES}

\section{Instructions:}

- Les filles forment une longue file ayant leurs jambes écartées pour former un tunnel. La dernière de la file doit passer par le tunnel jusqu'à ce qu'elle sorte devant, en faisant attention à ne pas heurter un partenaire et à ne pas se faire heurter elle-même.

- Elles continuent ainsi jusqu'à ce que toutes les filles soient passées.

- Cette activité leur permet de développer la confiance entre elles pendant qu'ils sont dans un espace sûr. 
"C'EST MA MAISON"

Ce jeu peut être joué avec un nombre illimité de participantes, qui forment un cercle.

\section{Instructions :}

Les participantes chantent la chanson suivante qui mentionne des parties d'une maison, et chacun d'entre elles doit pointer du doigt une partie du corps qui ressemble à la partie de la maison qui est citée.

C'est ma maison et c'est la maison du voisin.

C'est ma porte et c'est la porte du voisin.

C'est ma fenêtre et c'est la fenêtre du voisin.

... et ainsi de suite avec les parties du corps.

\section{Réflexion :}

- Le jeu offre un espace pour réfléchir au fait qu'il y a les parties du corps que nous permettons de toucher et d'autres que nous ne le permettons pas.

- L'un de nos droits sexuels est que personne ne peut nous toucher si nous ne le voulons pas.

- $\quad$ Si une fille ne veut pas jouer, elle en a le droit, jusqu'à ce qu'elle décide peu à peu de participer.

- Après le jeu, expliquez que chaque partie du corps a son propre nom et qu'après l'explication, elles doivent rejouer en utilisant les noms corrects.

Maison : corps entier

Porte : bouche

Les fenêtres : les yeux

Oreillers : seins

Cuisine : l'estomac

Ventilateur: fesses et anus

Robinet : urètre

\section{LOTERIE DU CORPS}

Objectif : Ce jeu a pour but de renforcer l'identification des parties du corps, de voir à quoi elles ressemblent et de voir comment elles sont écrites dans les différentes langues qu'une fille pourrait utiliser dans sa vie quotidienne.

\section{Instructions :}

- La loterie du corps se joue avec une carte qui présente des dessins de parties du corps avec leur nom écrit dans la langue locale (si pertinent) et dans la langue dominante.

- La mentore peut également appeler «loterie!», en utilisant des cartes des parties du corps.

\section{PASSEZ LE JETON}

Objectif : Le groupe est divisé en deux équipes alignées sur deux rangées. Le but du jeu est de passer de la compétition à la coopération et à la confiance.

\section{Instructions :}

- Dans la première phase du jeu, les filles doivent donner un jeton à la première personne de chaque rangée. Le jeton doit être mis entre leurs vêtements (commençant par le col du chemisier et jusqu'à sortir de la jambe du pantalon). Une fois fini, on passe le jeton au joueur suivant.

- L'équipe qui parvient à faire passer le jeton au dernier joueur de la rangée et qui le fait revenir au premier joueur de la rangée en premier gagne.

- Dans la deuxième phase du jeu, on demande aux filles de se regarder et de se donner la main pour former un pont.

- Un volontaire du groupe se couchera sur le dessus des bras avec l'intention d'atteindre le bout du pont uniquement par le mouvement des bras des filles. Son partenaire peut l'attendre à la fin du pont pour l'aider à descendre. On peut jouer ce jeu à plusieurs reprises s'il y a plus d'un volontaire.

- À la fin, demandez aux filles ce qu'elles ont ressenti à chaque phase, quel type de contact physique y a-t-il eu, comment se sont-elles senties et pourquoi se sont-elles senties ainsi ? Faites la différence avec les autres formes de contact physique qui les mettent mal à l'aise et qu'elles ont le droit de rejeter et de dénoncer pour y mettre fin. L'élimination de la concurrence entre elles permet aux filles de voir l'importance du soutien entre femmes. 


\section{JEUX ET EXERCICES PARTICIPATIFS (suite)}

Jeux pour promouvoir l'éducation à la santé sexuelle et reproductive

\section{JEU SUR LES RÔLES FAMILIAUX}

\section{Instructions :}

- Les filles forment deux groupes. Le groupe 1 est une famille de deux parents avec trois enfants, et le groupe 2 est une famille de deux parents et six enfants.

- La mentore distribue un morceau de tortilla (ou tout autre produit alimentaire local) à chaque groupe et leur demande de le partager entre les membres de la famille. Ensuite, elle donne les informations sur les morceaux de nourriture de chaque groupe.

- Les groupes réfléchissent à la question de savoir comment le nombre d'enfants qu'ils ont affecte les ressources qui peuvent être allouées à chacun d'entre eux.

- Ils considèrent que le planning familial ce sont les questions liées au nombre d'enfants, et au moyen de subvenir aux besoins de ses enfants. Avec les adolescentes, cela peut être une introduction au sujet sur les méthodes de planification familiale.

\section{CHASSE AU PAPIER}

\section{Instructions :}

- La mentore prépare sept papiers en forme de parties du corps d'une fille.

- Chaque papier est caché dans l'espace sûr.

- Formés en sept groupes, les participantes cherchent les papiers et les rassemblent pour assembler la silhouette d'un corps.

- Lorsqu'elles auront fini, elles nommeront les parties du corps, leurs fonctions, leurs besoins en soins, si elles peuvent être touchées ou non, entre autres commentaires.

\section{DÉS}

\section{Instructions :}

- Les mentores doivent préparer un dé en écrivant sur chaque côté une question liée au corps.

- Les filles forment un cercle et attendent que La mentore lance le dé. La participante qui l'attrape doit répondre à l'une des questions qui se trouvent sur le dé, puis elle le passe à une autre fille dans le cercle.

- Certaines questions peuvent être : quels sont les noms donnés aux différentes parties du corps?

\section{CHARADES}

Instructions :

- Formez deux groupes. Un groupe quitte la salle et l'autre reste à l'intérieur.

- La mentore explique que le groupe qui est sorti va présenter des charades au groupe à l'intérieur afin qu'il puisse les deviner.

- Les thèmes des charades peuvent varier; certains peuvent se rapporter aux droits sexuels et reproductifs, aux étapes de la vie reproductive, aux méthodes de planification familiale.

- La mentore précise le sujet et le groupe se met d'accord avant d'entrer pour le présenter à l'autre groupe.

- Quand le groupe est prêt, les filles entrent et disent «Nous arrivons! » L'autre groupe répond: "Quel message nous apportez-vous?"

- La charade est lancée, et les filles peuvent faire quatre propositions.

- $\quad$ Si elles ne le devinent pas, le groupe explique ce qu'il essayait de présenter. 


\section{L'HYGIÈNE DU CORPS}

\section{Objectif :}

Chanter une chanson qui incite les participantes à apprendre comment nettoyer les parties de leur corps.

\section{Instructions :}

La mentore assigne à chaque fille une partie du corps et lui demande de réfléchir à la façon dont elle la nettoie. La chanson peut être en français ou dans une langue locale.

Regardez comment je fais,

Tout le monde le fait de cette façon.

Faites le tour de chacune des filles, en mentionnant différentes parties du corps. Réfléchissez au fait qu'il est important de garder toutes les parties de son corps propre afin de ne pas tomber malade ou de ne pas rendre d'autres personnes malades.

Mains : frotter avec de l'eau et du savon.

Cheveux : laver, peigner et démêler.

Dents : se brosser les dents avec du dentifrice et une brosse à dents.

Vulve : se laver à l'eau et au savon.

Aisselles: se laver à l'eau et au savon.

\section{Réflexion :}

- Qu'est-ce que la menstruation?

- Comment ressentez-vous le fait que vous allez avoir vos menstruations?

- Mentionnez un droit sexuel que vous connaissez.

- Mentionnez un droit reproductif que vous connaissez.

\section{PILE OU FACE}

\section{Instructions :}

- La mentore apporte un jeton et le jette dans la l'air.

- Elle demande aux filles de choisir pile ou face.

- Celles qui ont donné la bonne réponse se rassemblent pour répondre à la question que la mentore leur posera.

- Ensemble, elles en discutent et répondent en groupe.

- La mentore dispose d'une liste de questions sur les parties du corps. Les questions peuvent être :

Qu'est-ce qu'un vagin?

Qu'est-ce que la menstruation?

Quelle est la fonction des ovaires?

Quelle est la fonction des trompes de Fallope?

Quelle est la fonction de l'utérus?

\section{STATUES EN MARBRE}

Instructions :

- Les filles sont en cercle et la mentore chante : les statues en marbre bougent ici, bougent là...

- Puis la mentore prononce le nom d'une partie du corps.

- À chaque fois qu'une partie est mentionnée, les filles doivent se positionner dans la forme de cette partie du corps.

\section{NOMMEZ UN CORPS}

\section{Instructions :}

- Formez deux équipes, et chaque équipe propose un volontaire.

- La mentore donne à chaque groupe des étiquettes avec les noms des parties du corps d'une fille à placer sur la fille au bon endroit.

- Le groupe qui finit le premier, gagne.

\section{CHANSON}

\section{Instructions :}

- Les participantes forment un cercle et la mentore lance une chanson qui nomme les parties du corps qui sont visibles.

- Les filles montrent les parties du corps.

- Ensuite, la mentore commence à citer les parties du corps qui ne sont pas visibles, de sorte que les filles reconnaissent qu'il y a des organes externes et des organes internes.

Dans ce monde, il y a beaucoup de petites filles (3 répétitions)

Qui ne connaissent pas leur corps (2 répétitions)

Je vais maintenant vous dire

Tête, épaules, genoux et orteils

Mon corps bouge, bouge comme je veux.

... dire la partie du corps 


\section{INITIATIVE POUR LES ADOLESCENTES-KENYA : ADAPTATION DU PROGRAMME POUR LES MENTORES FAIBLEMENT ALPHABÉTISÉES}

\begin{abstract}
L'Initiative pour les adolescentes au Kenya (AGI-K) a aidé les jeunes adolescentes vulnérables à se constituer un capital social, sanitaire et économique. L'AGI-K a testé des combinaisons d'interventions en matière de santé, de prévention de la violence, de création de richesse et d'éducation afin de déterminer quel ensemble d'interventions a le plus d'impact et à quel coût. Le programme a touché plus de 6000 filles âgées de 11 à 14 ans sur une période de deux ans à Kibera, un quartier urbain informel de Nairobi, et dans la région rurale de Wajir, une zone isolée et sous-développée du Kenya, le long de la frontière nord-est avec la Somalie.
\end{abstract}

\section{En raison des faibles taux}

d'alphabétisation et des variations dans I'expérience avec la facilitation sur le site AGI-K de Wajir, au nord du Kenya, un site rural isolé et mal desservi, le Population Council et ses partenaires d'exécution ont eu des difficultés à recruter et à retenir des mentores capables de faciliter le curriculum des espaces sûrs. Dans les six mois qui ont suivi le lancement de l'intervention, l'équipe du programme a reconnu qu'elle devait adapter le programme de Wajir pour répondre aux besoins des mentores faiblement alphabétisées.

Tout d'abord, ils ont transposé en scripts audio le curriculum écrit en anglais de Wajir Health and Life Skills et Financial Education, qui ont ensuite été traduits en langue somalienne. Puis, ils ont fait des enregistrements audio de chaque session, ont mis les sessions sur des clés USB et ont fourni à chaque mentore à Wajir une simple radio avec un port USB. Le coût de chaque radio et USB était d'environ 30 dollars par mentore.

Les sessions audio ont aidé les mentores à animer efficacement les séances, même si leur niveau d'alphabétisation était faible. Au-delà de résoudre la question de l'analphabétisme, les sessions audio ont permis d'ouvrir le débat entre les mentores et les participantes sur les questions liée à la SDSR, qui sont souvent évitées dans la communauté plus réservée de Wajir. De plus, les sessions audios ont permis de standardiser le contenu délivré puisque l'information était enregistrée sur un support fixe et qu'il ne revenait pas à la mentore individuelle de décider quoi dire ou ne pas dire lors de l'animation des sessions.

Bien qu'il y ait encore eu quelques variations d'une mentore à l'autre au cours des activités et des discussions, les enregistrements ont contribué à rendre la diffusion des informations plus uniforme. De plus, certains adultes de la communauté écoutaient les sessions audio après les sessions dans l'espace sûr, ce qui a contribué à dissiper certains mythes et suspicions sur ce dont les mentores et les filles discutaient dans les groupes.

Bien que l'utilisation de sessions audio n'ait pas été prévue au début de l'AGI-K, cette innovation s'est avérée être un outil efficace qui pourrait être utile à d'autres programmes communautaires dirigés par des mentores.

Pour plus d'informations: Austrian, K., E. Soler-Hampejsek, J. Mumah, B. Kangwana, Y.Wado, B. Abuya, V.Shah, and J. Maluccio. 2018. "Adolescent Girls Initiative-Kenya: Midline Results Report." Nairobi: Population Council. 


\section{CHAPITRE 4}

\section{Suivi et évaluation}

$\begin{array}{llr}\text { OUTIL } & \text { Suivi hebdomadaire } & \text { Page } 89 \\ \text { OUTIL } & \text { Suivi mensuel } & 90 \\ \text { OUTIL } & \text { Guide d'accompagnement } & 91 \\ \text { OUTIL } & \text { Liste de contrôle des observations } & 95 \\ \text { OUTIL } & \text { Formulaire d'évaluation des participantes } & 100 \\ & \end{array}$


Les mentores jouent un rôle central dans les programmes. Il est donc essentiel de suivre et d'évaluer les mentores individuelles et leurs systèmes de soutien et de gestion. Si le suivi et l'évaluation sont de plus en plus acceptés comme une nécessité plutôt que comme un complément facultatif, il est également important de souligner la nature responsabilisante de cette pratique. Le processus de suivi et d'évaluation est une occasion importante pour les mentores de renforcer ce qui fonctionne bien et de se développer de manière spécifique dans l'exercice de leurs fonctions.

Le suivi et l'évaluation des mentores prend de nombreuses formes, de l'évaluation quantitative à l'évaluation qualitative des processus de changement, en passant par des contrôles mensuels avec le personnel du programme pour discuter des questions émergentes. Certaines mentores et superviseurs de mentores utilisent des formulaires papier ou des méthodes visuelles pour suivre les présences, identifier les personnes nécessitant une attention particulière et d'autres questions ; les mentores de certains programmes utilisent des outils basés sur le téléphone portable. Ces outils doivent utiliser la méthode de collecte d'informations la plus simple et la plus facile à utiliser, qui correspond aux compétences des mentores.

Les retours d'information sur les performances des mentores proviennent de nombreuses sources - y compris des collègues mentores, des superviseurs, des observateurs extérieurs et des participantes au programme. Un principe clé est de ne pas collecter plus d'informations que le programme n'en utilisera.

Bien que les mentores soient généralement chargées de la mise en œuvre des programmes, l'expérience du mentorat enrichit souvent leur propre vie, renforce leurs atouts et leurs compétences, et peut même affecter leur bien-être psychologique; de cette façon, les mentores sont aussi des bénéficiaires du programme. Voici quelques exemples d'outils permettant d'évaluer la façon dont le mentorat change la vie des mentores.

Cette section comprend des outils et des guides pour suivre et soutenir les mentores et évaluer les performances. 


\section{SUIVI HEBDOMADAIRE}

Cet outil peut être utilisé par les superviseurs des mentores pour noter si un rencontre a eu lieu, si des données ont été soumises par chaque mentore, si la session concernée a été animée et si le curriculum a été suivi. Il peut également être utilisé pour déterminer si un faible taux de participation a été observé, et si un nombre élevé de filles non inscrites au programme était présent. Les mentores peuvent ainsi utiliser ces informations pour faire un suivi auprès des participantes afin de déterminer ce qui les empêche de participer aux sessions.

\begin{tabular}{|c|c|c|c|c|c|c|c|c|c|c|}
\hline $\begin{array}{l}\text { SE- } \\
\text { MAINE } \\
\text { DU }\end{array}$ & $\begin{array}{l}\text { Nom de la } \\
\text { mentore et } \\
\text { numéro du } \\
\text { groupe }\end{array}$ & $\begin{array}{l}\text { Données de } \\
\text { la rencontre } \\
\text { soumis }\end{array}$ & $\begin{array}{l}\text { Ren- } \\
\text { contre a } \\
\text { eu lieu }\end{array}$ & $\begin{array}{l}\text { Session } \\
\text { perti- } \\
\text { nente } \\
\text { facilitée }\end{array}$ & $\begin{array}{l}\text { Curriculum } \\
\text { suivi }\end{array}$ & $\begin{array}{l}\text { Faible taux } \\
\text { de partici- } \\
\text { pation } \\
\text { observé }\end{array}$ & $\begin{array}{l}\text { Nombre } \\
\text { élevé de } \\
\text { filles ne } \\
\text { participant } \\
\text { pas au } \\
\text { programme }\end{array}$ & Commentaires & $\begin{array}{l}\text { Besoins } \\
\text { de suivi }\end{array}$ & $\begin{array}{l}\text { Mesures } \\
\text { prises } \\
\text { après le } \\
\text { suivi }\end{array}$ \\
\hline [Date] & & & & & & & & & & \\
\hline [Date] & & & & & & & & & & \\
\hline [Date] & & & & & & & & & & \\
\hline [Date] & & & & & & & & & & \\
\hline [Date] & & & & & & & & & & \\
\hline [Date] & & & & & & & & & & \\
\hline [Date] & & & & & & & & & & \\
\hline [Date] & & & & & & & & & & \\
\hline [Date] & & & & & & & & & & \\
\hline [Date] & & & & & & & & & & \\
\hline [Date] & & & & & & & & & & \\
\hline [Date] & & & & & & & & & & \\
\hline [Date] & & & & & & & & & & \\
\hline [Date] & & & & & & & & & & \\
\hline [Date] & & & & & & & & & & \\
\hline
\end{tabular}




\section{SUIVI MENSUEL}

L'outil ci-dessous doit être rempli par les mentores et permet de réfléchir aux sessions animées au cours du mois précédent. Cet outil est issu d'un programme qui comprenait des bons de santé, des comptes d'épargne et qui mettait l'accent sur la violence basée sur le genre, mais d'autres activités pertinentes au programme peuvent être ajoutées selon les besoins.

\begin{tabular}{|c|c|}
\hline \multicolumn{2}{|l|}{ Nom } \\
\hline Site & \\
\hline Mois de référence & \\
\hline $\begin{array}{l}\text { SESSIONS COUVERTES DANS LES RENCONTRES D’ESPACES SÛRS (ES) } \\
\text { PENDANT LE MOIS DE REFÉRENCE }\end{array}$ & \\
\hline Réunion 1 & \\
\hline Réunion 2 & \\
\hline Réunion 3 & \\
\hline Réunion 4 & \\
\hline Ce que les filles ont le plus et le moins aimé dans les sessions de ce mois? & \\
\hline $\begin{array}{l}\text { Défis rencontrés lors de l'animation des sessions ou de la conduite des } \\
\text { réunions ES du mois }\end{array}$ & \\
\hline Réussites/réalisations des réunions ES de ce mois & \\
\hline BON DE SANTÉ & \\
\hline Nombre de bons de santé additionnels distribués ce mois-ci & \\
\hline Nombre de bons de santé déclarés perdus par les filles ce mois-ci & \\
\hline $\begin{array}{l}\text { Nombre de fois où la mentore a accompagné une fille à la clinique pour } \\
\text { utiliser un bon de santé }\end{array}$ & \\
\hline $\begin{array}{l}\text { Retour d'expérience sur l'accompagnement des jeunes filles à la clinique } \\
\text { pour l'utilisation de leurs bons de santé }\end{array}$ & \\
\hline $\begin{array}{l}\text { Commentaires des filles sur leur expérience de l'utilisation des bons de santé } \\
\text { (positifs et négatifs) }\end{array}$ & \\
\hline COMPTE D'ÉPARGNE & \\
\hline $\begin{array}{l}\text { Nombre de nouveaux comptes d'épargne ouverts au cours du mois de } \\
\text { référence }\end{array}$ & \\
\hline $\begin{array}{l}\text { Mise à jour sur les mesures prises pour résoudre les problèmes liés aux } \\
\text { comptes d'épargne en suspens }\end{array}$ & \\
\hline $\begin{array}{l}\text { Nombre de fois où la mentore a accompagné une fille à la banque pour } \\
\text { effectuer une transaction }\end{array}$ & \\
\hline $\begin{array}{l}\text { Retour d'expérience sur l'accompagnement des filles à la banque pour } \\
\text { l'utilisation de leurs comptes }\end{array}$ & \\
\hline $\begin{array}{l}\text { Commentaires des filles sur leur expérience dans l'utilisation de leur compte } \\
\text { d'épargne }\end{array}$ & \\
\hline Visites à domicile effectuées, y compris les résultats des visites & \\
\hline Nombre de cas de violence basée sur le genre (VBG) signalés & \\
\hline Détails des cas de VBG signalés et des mesures prises & \\
\hline Nombre de cartes d'identification du programme distribuées ce mois-ci & \\
\hline Nombre de cartes d'identification du programme perdues ce mois-ci & \\
\hline
\end{tabular}

Témoignages de filles pour le mois de référence (positifs et négatifs)

Qui (y compris son âge) :

Que s'est-il passé ? Qu'est-ce qui a conduit à cette situation (si vous le savez)?

Où la situation s'est-elle produite?

Comment la situation est-elle/a-t-elle été gérée ? Quel en a été le résultat ?

Des informations supplémentaires à partager concernant cette situation? 


\section{GUIDE D’ACCOMPAGNEMENT ${ }^{33}$}

Dans certains programmes, l'accompagnement de la mentore se fait lors de visites mensuelles dans les sessions dans l'espace sûr de la mentore et offre un retour d'information opportun pour améliorer la qualité. Les performances sont évaluées à l'aide d'indicateurs établis dans le guide d'accompagnement, suivi d'un dialogue avec la mentore pour réfléchir aux aspects les plus pertinents de ses performances, dans un processus qui offre l'opportunité d'apprendre.

Le guide d'accompagnement comporte quatre sections qui mettent l'accent sur ce que les participantes considèrent comme les meilleurs atouts du programme :

1. Gagner en confiance pour participer;

2. Augmenter l'estime de soi ;

3. Acquérir de nouvelles connaissances; et

4. Élargir le réseau d'amis.

Chaque section est divisée en plusieurs indicateurs qui sont classés par le « système de feux de circulation », décrivant le niveau de performance par des couleurs : vert, jaune ou rouge.

\section{Avant la visite}

- Confirmez la date et l'heure de la session avec la mentore.

- Identifiez le type de session qui aura lieu.

- S'il s'agit d'une session thématique, lisez les activités proposées dans le guide du programme.

- Informez la mentore de la communauté que vous allez visiter. (Vous pouvez également effectuer des visites surprises sans notification préalable.)

- Si ce n'est pas la première fois que vous rendez visite à la mentore, identifiez ses principales forces et faiblesses.

- Remplissez les données générales dans le formulaire du Guide d’accompagnement.

\section{Pendant la visite}

Il est important de limiter l'impact de la visite sur les activités habituelles et en cours. En arrivant dans la communauté :

- Saluez la mentore et les adolescentes de la communauté.

- Expliquez le but de la visite de suivi.

- Renforcez la confiance de la mentore de la communauté.

\section{Pendant la session}

- Saluez le groupe de participantes et dites que vous êtes encouragé à voir comment elles travaillent, jouent et parlent pendant la session.

- Prenez place dans l'espace sûr dans un endroit où vous pouvez voir le groupe et la mentore communautaire, sans interrompre les activités.

- Remplissez le formulaire du guide d'accompagnement en traçant un X dans la case de la colonne des feux de circulation qui décrit le mieux ce qui s'est passé pendant la séance.

- Prenez des notes, notez des suggestions, des questions, des idées et/ou des félicitations à ;a mentore dans la dernière colonne.

- II n'est pas nécessaire de remplir le formulaire dans l'ordre dans lequel il est présenté. Remplissez-le en fonction du moment où les situations pertinentes se présentent dans la session.

33 https://knowledgecommons.popcouncil.org/departments_sbsr-pgy/593/ 


\section{GUIDE D'ACCOMPAGNEMENT (suite)}

\section{Après la session}

- Félicitez la mentore en soulignant un aspect positif de sa mis en œuvre.

- Développez un dialogue avec la mentore sur la base du guide d'accompagnement.

- Demandez à la mentore : qu'est-ce que vous trouvez utile dans cette session ? Quels ont été les résultats des participantes? Quels objectifs restent en suspens?

- Partagez les résultats et les notes dans le formulaire du Guide d'accompagnement.

- Convenez de stratégies pour améliorer la qualité de la session, en abordant les aspects qui sont médiocres.

- Reconnaîssez l'ouverture d'esprit de la mentore et sa volonté de continuer à soutenir les adolescentes, en appréciant un autre aspect positif de sa performance.

\section{Après la visite}

- Il est important de noter les résultats dans les instruments de suivi immédiatement après la visite afin de ne pas perdre des informations précieuses, d'identifier toute lacune qui doit être examinée dès que possible avec la mentore, et de traiter avec le coordinateur du site toute question pertinente qui mérite d'être prise en compte.

- Saisissez les résultats dans la base de données de suivi appropriée.

- Transcrivez les commentaires en suspens de la visite.

- Déposez le formulaire dans les dossiers de la mentore.

- Communiquez à la partie concernée toute situation inhabituelle qui requiert une attention particulière. 


\begin{tabular}{|l|l|}
\hline Nom de la mentore & \\
\hline Date de la visite & \\
\hline Communauté visitée & \\
\hline Nombre de participantes présentes à la session & \\
\hline Activité prévue pour la journée & \\
\hline Heure de début de la session: & Heure de fin : \\
\hline État de l'espace sûr & \\
\hline
\end{tabular}

\section{La mentore mène les sessions.}

\begin{tabular}{|c|c|c|}
\hline $\begin{array}{l}\text { Donne des } \\
\text { instructions } \\
\text { claires et } \\
\text { guide les } \\
\text { activités }\end{array}$ & $\begin{array}{l}\text { Vert: Elle a dit avec assurance ce que le groupe devait faire et } \\
\text { les participantes l'ont compris. } \\
\text { Jaune: Elle a dit ce qu'il fallait faire, mais elle s'est } \\
\text { embrouillée et a donné des instructions à plusieurs reprises. } \\
\text { Rouge: Elle n'a pas dit ce qu'il fallait faire, le groupe ne l'a pas } \\
\text { comprise et elle a lu les activités directement du guide du } \\
\text { programme. }\end{array}$ & Notes: \\
\hline $\begin{array}{l}\text { Effectue les } \\
\text { activités } \\
\text { nécessaires } \\
\text { pour attein- } \\
\text { dre les } \\
\text { objectifs }\end{array}$ & $\begin{array}{l}\text { Vert: Elle a effectué les activités nécessaires pour atteindre les } \\
\text { objectifs. } \\
\text { Jaune: Elle a réalisé certaines activités, atteint certains objectifs, } \\
\text { mais pas tous. } \\
\text { Rouge: Elle n'a pas mené d'activités qui auraient permis } \\
\text { d'atteindre les objectifs de la session. }\end{array}$ & \\
\hline $\begin{array}{l}\text { Se présente à } \\
\text { la session avec } \\
\text { le matériel } \\
\text { nécessaire } \\
\text { déjà préparé }\end{array}$ & $\begin{array}{l}\text { Vert: Elle s’est présentée à la session avec le matériel nécessaire } \\
\text { déjà préparé. } \\
\text { Jaune: Elle est venue avec une partie du matériel préparé, mais il } \\
\text { manquait certaines choses. } \\
\text { Rouge: Elle est arrivée sans le matériel préparé pour la session. }\end{array}$ & \\
\hline $\begin{array}{l}\text { Comprend le } \\
\text { sujet sur } \\
\text { lequel le } \\
\text { groupe } \\
\text { travaille }\end{array}$ & $\begin{array}{l}\text { Vert: Elle a expliqué le sujet avec des informations correctes et a } \\
\text { su répondre aux questions. } \\
\text { Jaune: Elle a maîtrisé certaines informations, mais pas assez. } \\
\text { Rouge: Elle a fourni des informations erronées par rapport au } \\
\text { sujet et n'a pas su répondre aux questions. }\end{array}$ & \\
\hline \multicolumn{3}{|c|}{ 2. La mentore encourage la participation. } \\
\hline $\begin{array}{l}\text { Pose des } \\
\text { questions pour } \\
\text { identifier les } \\
\text { connaissances } \\
\text { préalables des } \\
\text { participantes }\end{array}$ & $\begin{array}{l}\text { Vert: Elle a posé des questions qui ont incité les participantes à } \\
\text { s'exprimer et à partager leurs idées sur le sujet. } \\
\text { Jaune: Les questions qu'elle a posées n'ont pas encouragé les } \\
\text { participantes à s'exprimer sur le sujet. } \\
\text { Rouge: Elle n'a pas posé de questions sur le sujet. }\end{array}$ & \\
\hline $\begin{array}{l}\text { Permet aux } \\
\text { participantes } \\
\text { de guider les } \\
\text { activités et de } \\
\text { prendre des } \\
\text { initiatives }\end{array}$ & $\begin{array}{l}\text { Vert: Elle a permis aux participantes de mener des activités pendant } \\
\text { la session. } \\
\text { Jaune: Les adolescentes ont participé aux activités, mais n'ont pas } \\
\text { animé d'activités. } \\
\text { Rouge: Elle n'a pas mené d'activités où les jeunes pouvaient } \\
\text { participer et être des leaders. }\end{array}$ & \\
\hline
\end{tabular}




\section{GUIDE D'ACCOMPAGNEMENT (suite)}

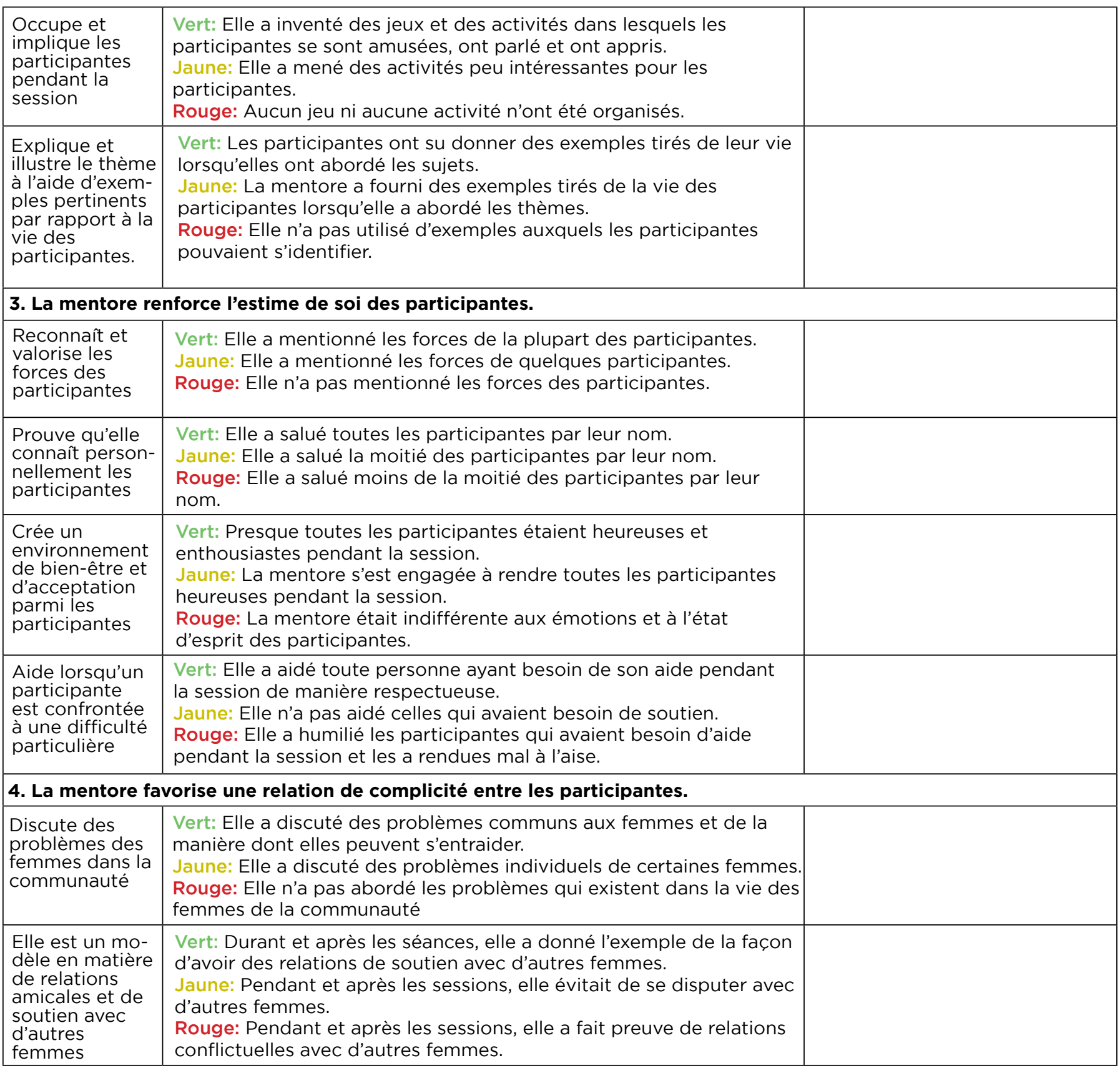




\section{LISTE DE CONTRÔLE DES OBSERVATIONS}

Cet outil mesure les performances des mentores en matière de compétences de facilitation, de création d'un environnement convivial, de communication, d'établissement de relations, de connaissances et de leadership. Les observateurs ( $y$ compris les assistants de recherche externes ou autres) effectuent une visite sur place et remplissent le formulaire, en indiquant le niveau de compétence sur la base du guide de notation décrit sous la liste de contrôle. ${ }^{34}$

Code de la mentore Site Date de la visite

Numéro du groupe Nombre de filles inscrites au groupe

Sujet/activité prévu(e) pour la journée Nombre de filles présentes

Session prévue de commencer à Session a commencé à Session s'est clôturé à

Lieu de l'espace sûr Évaluation réalisée par

Instructions : Cochez le niveau de compétence qui décrit le mieux les performances de la mentore.

\begin{tabular}{|c|c|c|c|c|c|c|}
\hline \multicolumn{2}{|r|}{ Facilitation } & Niveau 1 & Niveau 2 & Niveau 3 & N/A & Notes \\
\hline 1 & Patience & & & & & \\
\hline 2 & S'amuse avec les filles, est motivée & & & & & \\
\hline 3 & $\begin{array}{l}\text { Utilise des exemples pratiques pertinents } \\
\text { à la vie des filles }\end{array}$ & & & & & \\
\hline 4 & $\begin{array}{l}\text { Est empathique, attentionnée et sen- } \\
\text { sible : pose des questions afin d'identifier } \\
\text { les connaissances et les expériences des } \\
\text { filles sur le sujet de la session }\end{array}$ & & & & & \\
\hline 5 & $\begin{array}{l}\text { Crée un espace physique favorable et } \\
\text { propice à l'apprentissage }\end{array}$ & & & & & \\
\hline 6 & $\begin{array}{l}\text { Facilitation, gestion du temps : Utilise } \\
\text { le guide des sessions }\end{array}$ & & & & & \\
\hline 7 & $\begin{array}{l}\text { Préparation, gestion du temps: la } \\
\text { mentore atteint les objectifs fixés pour } \\
\text { la session en réalisant toutes les } \\
\text { activités }\end{array}$ & & & & & \\
\hline 8 & $\begin{array}{l}\text { Préparation, gestion } \\
\text { administrative : La mentore se } \\
\text { présente à la session préparée }\end{array}$ & & & & & \\
\hline
\end{tabular}




\section{LISTE DE CONTRÔLE DES OBSERVATIONS (suite)}

Instructions : Cochez le niveau de compétence qui décrit le mieux les performances de la mentore.

\begin{tabular}{|c|c|c|c|c|c|c|}
\hline \multicolumn{2}{|c|}{ Environment favorable } & \multirow[t]{2}{*}{ Niveau 1} & \multirow[t]{2}{*}{ Niveau 2} & \multirow[t]{2}{*}{ Niveau 3} & \multirow[t]{2}{*}{ N/A } & \multirow[t]{2}{*}{ Notes } \\
\hline 9 & $\begin{array}{l}\text { Création d'un espace émotionnellement } \\
\text { sûr, établissement de relations : Crée } \\
\text { une atmosphère de bien-être et } \\
\text { d'acceptation parmi les filles }\end{array}$ & & & & & \\
\hline 10 & $\begin{array}{l}\text { Création d'un espace émotionnellement } \\
\text { sûr, établissement de relations : Prouve } \\
\text { qu'elle connaît les filles personnellement }\end{array}$ & & & & & \\
\hline 11 & $\begin{array}{l}\text { Création d' un espace émotionnellement } \\
\text { sûr, ne pas porter de jugement, con- } \\
\text { struire des relations : discute des pro- } \\
\text { blèmes communs aux filles du groupe }\end{array}$ & & & & & \\
\hline \multicolumn{2}{|c|}{ Communication } & Niveau 1 & Niveau 2 & Niveau 3 & N/A & Notes \\
\hline 12 & $\begin{array}{l}\text { Communication/facilitation : } \\
\text { Recours au langage corporel pour } \\
\text { renforcer l'engagement des filles }\end{array}$ & & & & & \\
\hline 13 & $\begin{array}{l}\text { Communication/facilitation : Simplifie } \\
\text { le langage pour que les filles le } \\
\text { comprennent mieux }\end{array}$ & & & & & \\
\hline 14 & $\begin{array}{l}\text { Communication/facilitation : Donne des } \\
\text { instructions claires pendant la session }\end{array}$ & & & & & \\
\hline 15 & $\begin{array}{l}\text { Çommunication/facilitation: } \\
\text { Écoute active }\end{array}$ & & & & & \\
\hline \multicolumn{2}{|c|}{ Création de relations } & Niveau 1 & Niveau 2 & Niveau 3 & N/A & Notes \\
\hline 16 & Gestion de la colère & & & & & \\
\hline 17 & Résolution de conflits & & & & & \\
\hline 18 & $\begin{array}{l}\text { Travail d'équipe, établissement de } \\
\text { relations: Encourage le travail d'équipe } \\
\text { entre les filles }\end{array}$ & & & & & \\
\hline \multicolumn{2}{|c|}{ Connaissances } & Niveau 1 & Niveau 2 & Niveau 3 & N/A & Notes \\
\hline 19 & $\begin{array}{l}\text { Connaissance : Démontre une } \\
\text { compréhension du matériel }\end{array}$ & & & & & \\
\hline 20 & $\begin{array}{l}\text { Connaissance, développement des } \\
\text { talents des filles: Niveau de compré- } \\
\text { hension du matériel par les filles }\end{array}$ & & & & & \\
\hline \multicolumn{2}{|c|}{ Leadership } & Niveau 1 & Niveau 2 & Niveau 3 & N/A & Notes \\
\hline 21 & $\begin{array}{l}\text { Promouvoir le leadership : permet } \\
\text { aux filles de participer, de guider } \\
\text { des activités et d'assumer le rôle } \\
\text { de leadership }\end{array}$ & & & & & \\
\hline
\end{tabular}




\section{Guide d'évaluation}

\begin{tabular}{|c|c|c|c|c|}
\hline & & Niveau 1 & Niveau 2 & Niveau 3 \\
\hline \multicolumn{5}{|c|}{ Facilitation } \\
\hline 1 & Patience & $\begin{array}{l}\text { La mentore ne répond pas } \\
\text { aux questions des filles et } \\
\text { ne respecte pas leur rythme } \\
\text { de travail. }\end{array}$ & $\begin{array}{l}\text { La mentore s'adapte parfois à } \\
\text { leur rythme de travail et } \\
\text { répond soigneusement aux } \\
\text { questions des filles. }\end{array}$ & $\begin{array}{l}\text { La mentore répond toujours } \\
\text { attentivement aux questions/ } \\
\text { difficultés des filles et } \\
\text { s'adapte à leur rythme de } \\
\text { travail, ce qui leur permet de } \\
\text { se sentir bien dans leur peau. }\end{array}$ \\
\hline 3 & $\begin{array}{l}\text { Utilise des } \\
\text { exemples } \\
\text { pratiques } \\
\text { pertinents à la vie } \\
\text { des filles }\end{array}$ & $\begin{array}{l}\text { La mentore n'utilise pas } \\
\text { d'exemples lorsqu'elle } \\
\text { transmet des } \\
\text { informations. }\end{array}$ & $\begin{array}{l}\text { La mentore donne des } \\
\text { exemples concrets lors des } \\
\text { discussions sur un sujet. }\end{array}$ & $\begin{array}{l}\text { La mentore fournit des } \\
\text { exemples concrets et les } \\
\text { filles peuvent donner des } \\
\text { exemples de leur propre } \\
\text { vie tout en discutant du } \\
\text { sujet. }\end{array}$ \\
\hline 4 & $\begin{array}{l}\text { Est empathique, } \\
\text { attentionnée et } \\
\text { sensible: pose } \\
\text { des questions afin } \\
\text { de cerner les } \\
\text { connaissances et } \\
\text { les expériences } \\
\text { des filles sur le } \\
\text { sujet de la séance }\end{array}$ & $\begin{array}{l}\text { La mentore ne pose pas } \\
\text { de questions aux filles. }\end{array}$ & $\begin{array}{l}\text { La mentore pose des } \\
\text { questions qui ne stimulent } \\
\text { pas la participation et la } \\
\text { discussion. }\end{array}$ & $\begin{array}{l}\text { La mentore pose des } \\
\text { questions provocantes qui } \\
\text { stimulent la participation et } \\
\text { la discussion pour la } \\
\text { majorité des filles. }\end{array}$ \\
\hline 5 & $\begin{array}{l}\text { Crée un espace } \\
\text { physique } \\
\text { favorable et } \\
\text { propice à } \\
\text { l'apprentissage }\end{array}$ & $\begin{array}{l}\text { La mentore n'a apporté } \\
\text { aucune modification à } \\
\text { l'espace physique. }\end{array}$ & $\begin{array}{l}\text { La mentore a apporté des } \\
\text { modifications mineures à } \\
\text { l'espace physique - par } \\
\text { exemple en réorganisant } \\
\text { certains meubles pour créer } \\
\text { un environnement propice à } \\
\text { l'apprentissage en groupe. }\end{array}$ & $\begin{array}{l}\text { La mentore a entièrement } \\
\text { réorganisé l'espace } \\
\text { physique et son } \\
\text { emplacement physique, et a } \\
\text { pleinement mis à profit ses } \\
\text { mouvements dans l'espace. }\end{array}$ \\
\hline 7 & $\begin{array}{l}\text { Préparation, } \\
\text { gestion du temps: } \\
\text { La mentore atteint } \\
\text { les objectifs fixés } \\
\text { pour les sessions } \\
\text { de rencontre en } \\
\text { réalisant toutes les } \\
\text { activités }\end{array}$ & $\begin{array}{l}\text { La mentore n'atteint aucun } \\
\text { des objectifs de la session } \\
\text { de réunion. }\end{array}$ & $\begin{array}{l}\text { La mentore atteint certains } \\
\text { des objectifs généraux de la } \\
\text { session, mais les autres ne } \\
\text { sont pas atteints. }\end{array}$ & $\begin{array}{l}\text { La mentore atteint tous les } \\
\text { objectifs d'apprentissage } \\
\text { généraux de la session. }\end{array}$ \\
\hline
\end{tabular}




\section{LISTE DE CONTRÔLE DES OBSERVATIONS (suite)}

\section{Guide d'évaluation}

\begin{tabular}{|c|c|c|c|c|}
\hline 8 & $\begin{array}{l}\text { Préparation, } \\
\text { gestion } \\
\text { administrative : La } \\
\text { mentore se } \\
\text { présente à la } \\
\text { session préparée }\end{array}$ & $\begin{array}{l}\text { La mentore est venue sans } \\
\text { matériel pour la session et } \\
\text { n'a pas planifié la session à } \\
\text { l'avance. }\end{array}$ & $\begin{array}{l}\text { La mentore est venue avec } \\
\text { une partie du matériel et une } \\
\text { partie de la session planifiée. }\end{array}$ & $\begin{array}{l}\text { La mentore est venue avec } \\
\text { tout le matériel prêt et } \\
\text { semblait avoir planifié sa } \\
\text { session à l'avance. }\end{array}$ \\
\hline & & Niveau 1 & Niveau 2 & Niveau 3 \\
\hline \multicolumn{5}{|c|}{ Environnement favorable } \\
\hline 9 & \begin{tabular}{|l|} 
Création d'un \\
espace émotion- \\
nellement sûr, \\
création de \\
relations: Crée \\
une atmosphère \\
de bien-être et \\
d'acceptation
\end{tabular} & $\begin{array}{l}\text { La mentore mène la séance } \\
\text { sans tenir compte de } \\
\text { l'humeur ou des sentiments } \\
\text { des filles. }\end{array}$ & $\begin{array}{l}\text { La mentore reconnaît les } \\
\text { filles qui manquent } \\
\text { d'intérêt, mais ne cherche } \\
\text { pas à les aider. }\end{array}$ & $\begin{array}{l}\text { La mentore reconnaît les filles } \\
\text { qui ne sont pas intéressées } \\
\text { par la session et prend des } \\
\text { mesures pour les faire } \\
\text { participer. La majorité des } \\
\text { filles sont intéressées à } \\
\text { assister à la session et à } \\
\text { participer aux activités. }\end{array}$ \\
\hline 10 & $\begin{array}{l}\text { Création d'un } \\
\text { espace émotion- } \\
\text { nellement sûr, et } \\
\text { de relations: } \\
\text { Prouve qu'elle } \\
\text { connaît les filles } \\
\text { personnellement }\end{array}$ & $\begin{array}{l}\text { La mentore mentionne le } \\
\text { nom de moins de la moitié } \\
\text { des filles. }\end{array}$ & $\begin{array}{l}\text { La mentore prononce au } \\
\text { moins la moitié des noms } \\
\text { des filles. }\end{array}$ & $\begin{array}{l}\text { La mentore prononce le } \\
\text { nom de toutes les filles. }\end{array}$ \\
\hline 11 & $\begin{array}{l}\text { Création d'un } \\
\text { espace émotion- } \\
\text { nellement sûr, sans } \\
\text { jugement, propice } \\
\text { à la création de } \\
\text { relations: Discute } \\
\text { des problèmes } \\
\text { communs }\end{array}$ & $\begin{array}{l}\text { Les filles ne réfléchissent } \\
\text { pas aux problèmes } \\
\text { communs à leur vie. }\end{array}$ & $\begin{array}{l}\text { Les filles réfléchissent aux } \\
\text { problèmes qu'elles } \\
\text { rencontrent en tant } \\
\text { qu'individus, ou que des filles } \\
\text { comme elles rencontrent } \\
\text { dans la communauté. }\end{array}$ & $\begin{array}{l}\text { Les filles réfléchissent à leurs } \\
\text { problèmes en tant } \\
\text { qu'individus, ou à ceux des } \\
\text { filles de la communauté en } \\
\text { général, et partagent des } \\
\text { idées sur la manière de se } \\
\text { soutenir mutuellement. }\end{array}$ \\
\hline \multicolumn{5}{|c|}{ Communication } \\
\hline 12 & $\begin{array}{l}\text { Communication/ } \\
\text { Facilitation : Utili- } \\
\text { sation du langage } \\
\text { corporel pour } \\
\text { renforcer l'engage- } \\
\text { ment des filles }\end{array}$ & $\begin{array}{l}\text { La mentore n'a procédé à } \\
\text { aucun ajustement de son } \\
\text { langage corporel. }\end{array}$ & $\begin{array}{l}\text { La mentore a utilisé des } \\
\text { expressions faciales } \\
\text { engageantes et a modifié sa } \\
\text { posture. }\end{array}$ & $\begin{array}{l}\text { La mentore a ajusté ses } \\
\text { expressions faciales, sa } \\
\text { posture, ses gestes et le ton } \\
\text { de sa voix - tout cela pour } \\
\text { encourager la participation } \\
\text { des filles. }\end{array}$ \\
\hline 13 & $\begin{array}{l}\text { Communication/ } \\
\text { Facilitation : Sim- } \\
\text { plifie le langage } \\
\text { pour que les filles } \\
\text { comprennent }\end{array}$ & $\begin{array}{l}\text { La mentore n'a pas } \\
\text { cherché à simplifier la } \\
\text { langue utilisée dans le } \\
\text { programme. }\end{array}$ & $\begin{array}{l}\text { La mentore a parfois } \\
\text { simplifié le langage du } \\
\text { programme et a modifié le } \\
\text { ton de sa voix. }\end{array}$ & $\begin{array}{l}\text { La mentore a toujours } \\
\text { simplifié le langage du } \\
\text { programme et a modifié le } \\
\text { ton de sa voix afin } \\
\text { d'améliorer la participation } \\
\text { des filles. }\end{array}$ \\
\hline 14 & $\begin{array}{l}\text { Communication/ } \\
\text { Facilitation: Donne } \\
\text { des instructions } \\
\text { claires pendant la } \\
\text { session }\end{array}$ & $\begin{array}{l}\text { La mentore n'a pas } \\
\text { donné d'instructions } \\
\text { claires et n'a pas } \\
\text { expliqué davantage. }\end{array}$ & $\begin{array}{l}\text { La mentore a donné des } \\
\text { instructions peu claires, mais } \\
\text { a finalement réussi à faire } \\
\text { comprendre aux filles en } \\
\text { élaborant. }\end{array}$ & $\begin{array}{l}\text { La mentore a guidé avec } \\
\text { assurance le groupe à travers } \\
\text { les activités et les filles ont } \\
\text { compris. }\end{array}$ \\
\hline 15 & $\begin{array}{l}\text { Communication/ } \\
\text { Facilitation: } \\
\text { Écoute active }\end{array}$ & $\begin{array}{l}\text { La mentore n'a pas utilisé } \\
\text { de techniques d'«écoute } \\
\text { active ». }\end{array}$ & $\begin{array}{l}\text { La mentore a parfois utilisé } \\
\text { des expressions courtes et } \\
\text { encourageantes pour montrer } \\
\text { sa compréhension, et a } \\
\text { résumé les informations } \\
\text { apprises par les filles. }\end{array}$ & $\begin{array}{l}\text { La mentore a constamment } \\
\text { utilisé des expressions } \\
\text { encourageantes pour } \\
\text { résumer les sujets, a posé } \\
\text { des questions et a écouté } \\
\text { attentivement. }\end{array}$ \\
\hline
\end{tabular}




\begin{tabular}{|c|c|c|c|c|}
\hline & & Niveau 1 & Niveau 2 & Niveau 3 \\
\hline \multicolumn{5}{|c|}{ Création de relations } \\
\hline 16 & $\begin{array}{l}\text { Gestion de la } \\
\text { colère }\end{array}$ & $\begin{array}{l}\text { La mentore a répondu } \\
\text { violemment (verbalement } \\
\text { ou physiquement) et a } \\
\text { humilié les filles. }\end{array}$ & $\begin{array}{l}\text { La mentore n'a pas été } \\
\text { violent envers les filles, mais } \\
\text { a crié de façon inappropriée } \\
\text { ou leur a manqué de respect. }\end{array}$ & $\begin{array}{l}\text { La mentore a réagi d'une } \\
\text { manière qui a permis de } \\
\text { maîtriser la situation, tout } \\
\text { en respectant et en gardant } \\
\text { à l'esprit l'intérêt des filles. }\end{array}$ \\
\hline 18 & $\begin{array}{l}\text { Travail d'équipe, } \\
\text { renforcement } \\
\text { des relations: } \\
\text { Stimule le travail } \\
\text { d'équipe chez les } \\
\text { filles }\end{array}$ & $\begin{array}{l}\text { La mentore a encouragé } \\
\text { une compétition malsaine } \\
\text { entre les filles. }\end{array}$ & $\begin{array}{l}\text { La mentore n'a pas } \\
\text { encouragé les filles à } \\
\text { travailler ensemble. }\end{array}$ & $\begin{array}{l}\text { La mentore a encouragé } \\
\text { les filles à travailler } \\
\text { ensemble. }\end{array}$ \\
\hline \multicolumn{5}{|c|}{ Connaissances } \\
\hline 19 & $\begin{array}{l}\text { Connaissances: } \\
\text { Démontre une } \\
\text { compréhension } \\
\text { du matériel }\end{array}$ & $\begin{array}{l}\text { La mentore ne comprenait } \\
\text { pas le programme et ne } \\
\text { savait pas répondre aux } \\
\text { questions des filles. }\end{array}$ & $\begin{array}{l}\text { La mentore comprenait } \\
\text { partiellement le programme } \\
\text { et pouvait répondre aux } \\
\text { questions les plus } \\
\text { importantes des filles. }\end{array}$ & $\begin{array}{l}\text { La mentore a parfaitement } \\
\text { compris le programme, et a } \\
\text { su transmettre les } \\
\text { informations et répondre aux } \\
\text { questions des filles. }\end{array}$ \\
\hline 20 & $\begin{array}{l}\text { Connaissance, } \\
\text { développement } \\
\text { des talents des } \\
\text { filles: Niveau de } \\
\text { compréhension } \\
\text { du matériel par les } \\
\text { filles }\end{array}$ & $\begin{array}{l}\text { Les filles ne semblaient pas } \\
\text { comprendre le programme. }\end{array}$ & $\begin{array}{l}\text { Les filles ont partiellement } \\
\text { compris le programme et ont } \\
\text { su répondre aux questions les } \\
\text { plus importantes. }\end{array}$ & $\begin{array}{l}\text { Les filles ont parfaitement } \\
\text { compris le programme, et ont } \\
\text { été capables de communi- } \\
\text { quer avec les mentores et ont } \\
\text { su répondre aux questions de } \\
\text { manière claire et précise. }\end{array}$ \\
\hline \multicolumn{5}{|c|}{ Leadership } \\
\hline
\end{tabular}




\section{FORMULAIRE D’ÉVALUATION DES PARTICIPANTES}

Cet outil doit être rempli par les participantes au programme afin de donner aux filles la possibilité de donner leur avis sur leur mentore. II doit être clair que la mentore ne saura pas quelle fille a donné quelles réponses, afin que les filles puissent répondre sans craindre que cela ne change leur relation avec la mentore.

Site: Identifiant de la fille :
Nom et code de la mentore: Date de l'entretien :

\begin{tabular}{|c|c|c|c|c|}
\hline Questions & $\begin{array}{l}\text { D'AC- } \\
\text { CORD }\end{array}$ & $\begin{array}{l}\text { PAS D'AC- } \\
\text { CORD }\end{array}$ & $\begin{array}{l}\text { NE SAIS } \\
\text { PAS }\end{array}$ & $\begin{array}{l}\text { PAS } \\
\text { APPLICABLE }\end{array}$ \\
\hline \multicolumn{5}{|l|}{ Ma mentore se met parfois en colère contre les filles du groupe. } \\
\hline \multicolumn{5}{|l|}{$\begin{array}{l}\text { Ma mentore prend toujours le temps de répondre à toutes les } \\
\text { questions des filles. }\end{array}$} \\
\hline \multicolumn{5}{|l|}{$\begin{array}{l}\text { Lorsque j'arrive aux réunions de l'espace sûr, ma mentore est } \\
\text { généralement déjà là. }\end{array}$} \\
\hline \multicolumn{5}{|l|}{ J'aimerais être comme ma mentore quand je serai plus âgée. } \\
\hline \multicolumn{5}{|l|}{ Ma mentore n'est pas respecté dans la communauté. } \\
\hline \multicolumn{5}{|l|}{ Je ne fais pas confiance à ma mentore. } \\
\hline \multicolumn{5}{|l|}{ Ma mentore facilite la participation de toutes les filles du groupe. } \\
\hline \multicolumn{5}{|l|}{ Mes réunions dans l'espace sûr sont plaisantes. } \\
\hline \multicolumn{5}{|l|}{ Nous faisons des jeux éducatifs lors des sessions de groupe. } \\
\hline \multicolumn{5}{|l|}{$\begin{array}{l}\text { Parfois, nous nous répartissons en petits groupes pour discuter } \\
\text { de sujets lors de réunions de l'espace sûr. }\end{array}$} \\
\hline \multicolumn{5}{|l|}{ Ma mentore invite toujours les mêmes filles à participer. } \\
\hline \multicolumn{5}{|l|}{$\begin{array}{l}\text { Mon groupe d'espace sûr est un endroit où les filles se sentent } \\
\text { libres de poser toutes sortes de questions. }\end{array}$} \\
\hline \multicolumn{5}{|l|}{$\begin{array}{l}\text { Si j'avais un problème, ma mentore est l'une des premières } \\
\text { personnes à qui je le dirais. }\end{array}$} \\
\hline \multicolumn{5}{|l|}{$\begin{array}{l}\text { Lorsqu'il y a des problèmes entre filles dans le groupe de l'espace } \\
\text { sûr, ma mentore les aide à les résoudre. }\end{array}$} \\
\hline \multicolumn{5}{|l|}{$\begin{array}{l}\text { Ma mentore vient me voir après avoir manqué quelques réunions } \\
\text { de l'espace sûr. }\end{array}$} \\
\hline \multicolumn{5}{|l|}{$\begin{array}{l}\text { Lorsqu'il y a des problèmes avec les parents, ma mentore est } \\
\text { généralement en mesure d'aider à les résoudre. }\end{array}$} \\
\hline \multicolumn{5}{|l|}{$\begin{array}{l}\text { Ma mentore sait généralement répondre aux questions sur les } \\
\text { sujets qu'elle enseigne. }\end{array}$} \\
\hline \multicolumn{5}{|l|}{$\begin{array}{l}\text { Lorsque les filles posent des questions personnelles au sein du } \\
\text { groupe, les autres filles se moquent d'elles. }\end{array}$} \\
\hline \multicolumn{5}{|l|}{$\begin{array}{l}\text { Ma mentore aime certaines filles de notre groupe plus que } \\
\text { d'autres. }\end{array}$} \\
\hline \multicolumn{5}{|l|}{$\begin{array}{l}\text { Ma mentore dit que le planning familial est réservé aux filles } \\
\text { mariées. }\end{array}$} \\
\hline $\begin{array}{l}\text { Si je disais à ma mentore quelque chose de privé, elle le dirait à } \\
\text { quelqu'un d'autre }\end{array}$ & & & & \\
\hline
\end{tabular}




\section{Annexes}

$\begin{array}{ll}\text { 1. Foire aux questions } & \text { Page } 102\end{array}$

2. Description des programmes du Population Council 105

3. Liens vers les curriculums complets des programmes inclus dans 109 la boîte à outils de la mentore

4. Ressources et lectures supplémentaires 


\section{ANNEXE 1. FOIRE AUX QUESTIONS}

Nous avons interrogé le personnel du Population Council dans le monde entier qui gère les programmes pour les filles dirigés par des mentores et leur avons demandé de nous indiquer les questions fréquemment posées. Ces questions et leurs réponses sont les ci-dessous.

\section{Rémunération \\ Pourquoi payez-vous les mentores?}

Parce que nous leur demandons de faire un travail et qu'il n'est ni réaliste ni raisonnable, d'attendre une participation régulière et de qualité de la part d'une personne qui fait don de son temps. Vous devez payer les mentores pour obtenir un engagement de qualité dans le programme. La rémunération est un facteur de motivation pour que les mentores obtiennent de bons résultats. De plus, cela aide les mentores à surmonter la pression qu'elles peuvent ressentir pour se marier ou s'engager dans un travail dangereux pour soutenir leur famille.

\section{Comment décidez-vous du montant à payer?}

Les mentores doivent recevoir un salaire comparable à celui des postes ayant des exigences et des fonctions similaires sur le marché local, par exemple un agent de santé communautaire.

\section{Les mentores reçoivent-elles toutes le même salaire?} Les mentores qui effectuent les mêmes tâches reçoivent le même salaire. Dans certains cas, les mentores qui prennent en charge un plus grand nombre de groupes que la moyenne ou qui effectuent des tâches supplémentaires sont mieux rémunérés. Les mentores peuvent bénéficier d'une augmentation après un an de mentorat. Le fait que les mentores puissent ou non s'attendre à recevoir une augmentation de salaire peut faire partie du contrat de mentorat.

\section{Que pouvez-vous donner aux mentores en plus de leur rémunération?}

$\mathrm{Si}$ vous souhaitez accorder une rémunération supplémentaire aux mentores, vous pouvez envisager un soutien qui les aiderait à mieux faire leur travail, comme des vélos pour faciliter le déplacement entre les rencontres, des badges à montrer aux membres de la communauté lorsqu'elles recrutent des filles ou organisent des événements, des bottes de pluie pour la saison des pluies, etc. Certains programmes fournissent des uniformes ou une carte d'identité, le remboursement des frais de transport, des crédits pour le téléphone portable, des certificats d'achèvement et d'autres incitations. II appartient à chaque programme de décider ce qui sera utile et faisable.
Trouver de bons mentores Comment recruter des mentores?

Le personnel du programme crée une annonce d'emploi et l'affiche/diffuse dans la communauté, la transmet aux directeurs d'école et aux ONG de la communauté, et fait de la publicité dans des endroits comme le journal local. Ensuite, ils interviewent les candidates et en forment un plus grand nombre que requis afin que, si des problèmes apparaissent avec certaines candidates au cours de la formation, ces personnes puissent être éliminées.

\section{Quelles qualifications recherchez-vous?}

II n'y a pas de critère unique, car chaque programme et chaque environnement est unique. Le personnel du programme essaie de recruter des mentores qui ont terminé leurs études secondaires, qui savent lire et écrire et qui ont une certaine expérience avec les questions liées à la SDSR et à la facilitation. Idéalement, elles ont des attitudes "progressistes » sur la sexualité des adolescents, mais cela est souvent difficile à évaluer dans un entretien. Les mentores doivent être motivées et s'engager pour les filles. Certains programmes recherchent des mentores particulièrement dynamiques ou dotées d'une bonne maîtrise technologique. Les mentores doivent avoir le temps nécessaire à consacrer au programme, et ne doivent pas être susceptibles de quitter la communauté pendant la durée du programme.

Quel est le profil requis pour les mentores? Jeunes femmes âgées de 18 à 30 ans (en général), résidant dans la communauté dans laquelle elles assureront le mentorat (ou à proximité), diplômées de l'enseignement secondaire (si possible), maîtrisant la langue locale et disposées à voyager (dans les communautés et dans d'autres villes pour le programme de formation) pendant la durée du programme.

\section{Quelles sont les parties les plus importantes des} critères de sélection?

Selon les compétences requises par le programme, le niveau d'éducation peut être important, et la plupart des programmes estiment que la capacité et la motivation à travailler avec les filles sont plus importantes que l'expérience préalable. 
Que faire si vous ne pouvez pas trouver de mentores qui remplissent les conditions de qualification? Dans la plupart des communautés, vous trouverez des filles ou des jeunes femmes qui remplissent les conditions les plus importantes pour être mentores, bien qu'il faille parfois travailler davantage pour les trouver dans certains endroits. Dans certaines situations particulièrement difficiles, les exigences peuvent être étendues ou le programme peut être modifié en changeant le contenu pour l'adapter au contexte.

Je suis au-dessus de la tranche d'âge indiquée pour les mentores, mais je suis passionnée par le travail avec les adolescentes; puis-je être considérée comme une mentore?

Oui, vous pouvez être pris en considération, mais votre candidature sera examinée en fonction de votre expérience antérieure dans ce type de programme, ou dans une situation où nous n'avons pas suffisamment d'autres candidates appropriées.

\section{Que se passe-t-il si une mentore tombe enceinte?}

Le fait de tomber enceinte n'est pas un problème en soi, et cela ne signifie pas que la mentore doit être expulsée du programme ou puni. Les mentores peuvent choisir de rester dans le programme, et le personnel du programme peut les aider en leur apportant le soutien supplémentaire nécessaire pour poursuivre le mentorat. L'important est de maintenir la discussion avec la mentore sur la grossesse et d'avoir un cadre basé sur les droits pour faire ses propres choix. À la fin de la grossesse et juste après la naissance, il est préférable qu'une autre mentore prenne en charge les rencontres hebdomadaires du groupe afin que le programme ne soit pas interrompu.

\section{Formation}

\section{Quelle formation offrez-vous?}

La plupart des programmes prévoient une formation initiale des mentores, généralement d'une à deux semaines, suivie de rencontres mensuell des mentores qui servent de bilan et offre la possibilité de suivre une mini-formation de perfectionnement sur des sujets difficiles. Les possibilités de simulations de sessions de formation où les mentores utilisent des jeux de rôle pour apprendre les unes des autres sont une composante importante de la formation. La formation des mentores doit comporter des instructions spécifiques, étape par étape, sur la manière de mener les activités. Les évaluations des connaissances avant et après la formation sont d'excellents outils pour permettre aux mentores de voir tout ce qu'elles peuvent apprendre en une seule formation, ce qui peut renforcer leur estime de soi et leur motivation.
Quelle doit être la durée d'un atelier de formation? N'en faites pas trop; les ateliers de formation doivent être suffisamment longs pour couvrir les objectifs et le contenu, mais suffisamment courts pour que les mentores restent engagées et préparées jusqu'au prochain rencontre. Les formations doivent être amusantes, intéressantes et engageantes. La formation doit être un modèle du type de techniques d'animation participative attendues des mentores lorsqu'elles organisent leurs sessions de groupes de filles. Les mentores auront également besoin de temps pour se reposer et s'échanger lors de leur temps libre. Au début, elles peuvent avoir besoin d'activités prédéfinies pour interagir socialement. Au Guatemala, par exemple, les ateliers durent entre trois et quatre jours et comprennent des films éducatifs, des orateurs invités, des visites de musées, etc. Au cours de la première formation, ils couvrent le contenu des premiers mois et étudient le contenu pendant au moins les trois mois de sessions suivantes. Les mentores se réunissent après quelques mois pour un rencontre local au cours duquel elles étudient le contenu des sessions jusqu'à la prochaine formation.

J'ai suivi une formation similaire dans le cadre de mon précédent travail bénévole; dois-je encore assister toutes les sessions de formation des mentores?

Oui, vous devez assister à toutes les sessions inscrites à l'ordre du jour. Même si les programmes peuvent sembler similaires, certains éléments seront différents. Vous devez vous assurer que vous êtes bien équipé pour le travail.

Contexte culturel

Qu'en est-il de l'adaptation aux différents contextes sociaux et culturels?

Les mentores doivent comprendre les objectifs de chaque session et la pertinence de chaque activité suggérée, c'est-à-dire non seulement ce que la session vise à réaliser, mais aussi comment elle est pertinente par rapport à la vie des filles. Une étape pour les mentores consiste à planifier les sessions de manière indépendante, comme elles le feront dans leurs communautés, de les adapter aux besoins locaux, de les traduire en langues locales, de les illustrer par des exemples, etc. 


\section{Est-ce que parler de la santé sexuelle et reproductive est un tabou pour les mentores?}

Oui, souvent au début. Généralement, les sujets relatifs à la santé sexuelle et reproductive sont abordés dès la première session de formation par le biais de diverses activités et ressources. II est essentiel que les mentores se sentent à l'aise pour aborder ces sujets avant de pouvoir en parler à des groupes de filles. (Il est également crucial que les personnes qui dirigent la formation soient à l'aise.) L'attitude des mentores sur le contenu est importante : étant donné que tout le contenu du programme passe par la mentore avant d'atteindre les filles, si les mentores n'y croient pas, même le meilleur programme ne sera pas utile. Les questions de santé sexuelle et reproductive peuvent être difficiles à comprendre, il est donc important de les revoir plusieurs fois au cours du programme.

\section{Assurer l'engagement des mentores}

Quels sont les éléments importants pour maintenir les mentores dans le programme?

La rémunération est un élément clé, car elle permet d'alléger la pression que les mentores reçoivent des familles s'ils contribuent moins aux tâches ménagères en raison de leur travail de mentore. Les mentores doivent également savoir que leur travail est reconnu et apprécié. La formation continue peut permettre aux mentores de rester engagées dans leur communauté, et les autres mentores constituent un réseau amical, ce qui permet de maintenir la motivation des membres. Une approche flexible fondée sur les droits peut également être utile. Par exemple, si les mentores sont enceintes ou ont des enfants, elles devraient être autorisées à les emmener en formation. Certains mentores vont inévitablement changer d'emploi ou abandonner le programme parce que leur famille déménage ou qu'elles se marient. Il est bon d'avoir un groupe de mentores formées qui peuvent prendre la relève au cas où les mentores quitteraient le programme, afin d'éviter de perturber le programme.
Suivi, évaluation, supervision et soutien Comment puis-je suivre le travail des mentores? Les mentores peuvent être suivies de différentes manières pour atteindre différents objectifs. Des outils de suivi et des contrôles réguliers peuvent déterminer si les filles assistent aux rencontres, si l' "espace sûr » est perçu comme tel et si la mentore suit le programme d'études comme prévu. Les filles peuvent également fournir un retour d'information sur leurs mentores. Lors des rencontres périodiques, les mentores peuvent partager leurs difficultés et leurs succès. Même les programmes faisant l'objet d'un suivi intensif ne peuvent pas fournir un retour d'information constant. Les programmes peuvent compenser en créant des opportunités pour les mentores d'apprendre les unes des autres.

Comment les programmes peuvent-ils garantir que les mentores bénéficient du soutien dont elles ont besoin? Les mentores ont besoin d'une supervision continue pour bien faire leur travail. L'une des clés est de mettre en place un système de référencement solide afin que les mentores sachent quand elles doivent demander l'aide d'un superviseur ou se mettre en contact avec d'autres services disponibles dans la communauté pour les participantes ou pour elles-mêmes. Lors de la formation initiale, les mentores peuvent établir une liste des services de soutien disponibles dans leur communauté, tels que les services de santé sexuelle et reproductive de la clinique locale, le soutien psychosocial, les unités de soutien aux victimes de la police et les systèmes d'orientation en cas de violence basée sur le genre. Les informations sur les services disponibles et leur localisation sont essentielles tant pour les mentores que pour les filles des groupes. Tout le travail ne doit pas être laissé à la mentore - dans les situations difficiles (comme les conflits avec les parents ou employeurs), ils peuvent avoir besoin de la contribution du personnel du programme.

\section{Est-il injuste de rendre visite aux mentores alors} qu'elles ne s'y attendent pas?

Le but d'une visite non annoncée n'est pas de surprendre la mentore, mais d'évaluer ses performances lors d'une session typique, afin que les superviseurs puissent lui fournir des conseils de soutien basés sur ce qui va bien et sur la manière de s'améliorer. Si les mentores savent que le personnel du programme vient, elles peuvent se préparer plus que d'habitude, de sorte que la session ne serait pas le reflet fidèle de leur préparation habituelle et de leurs compétences d'animation. Le personnel du programme doit savoir si les mentores se préparent aux réunions et comment elles mènent leurs activités afin de fournir un retour d'information pour l'amélioration des performances. 


\section{ANNEXE 2. DESCRIPTION DES PROGRAMMES DE POPULATION COUNCIL}

\begin{abstract}
Abriendo Oportunidades (Ouvrir des opportunités)
Le Population Council, en collaboration avec des partenaires locaux et internationaux, a lancé Abriendo Oportunidades au Guatemala en 2004. Le programme s'adresse aux jeunes filles indigènes mayas de 8-9 ans et leur offre des réseaux de soutien social, des modèles et des mentores, des conseils sur la vie et le leadership, ainsi qu'une formation et une expérience

professionnelles pratiques pour les aider à réussir les transitions vers l'adolescence. Le programme fait appel à des leaders communautaires et forme des filles à la gestion de groupes communautaires de filles : des espaces sûrs où elles acquièrent des compétences pratiques et assument des rôles de leadership.
\end{abstract}

Les évaluations réalisées en 2007 et 2011 ont révélé des résultats positifs tant pour les mentores que pour les filles participantes, notamment:

- Atteinte de niveaux d'éducation plus élevés chez les mentores par rapport aux statistiques nationale

- Les mentores sont plus susceptibles de rester célibataires et sans enfant par rapport à la moyenne nationale des filles de leur âge

- Plus d'autonomie et d'auto-efficacité chez les filles participantes

Le programme a débuté dans une poignée de communautés rurales au Guatemala et s'est depuis élargi à l'échelle nationale, ainsi qu'au Belize et au Mexique (où le programme est appelé Abriendo Futuros). Le programme s'est développé pour inclure le tutorat et est en cours d'adaptation pour les filles des zones urbaines et pour les garçons. Le programme travaille également avec des partenaires dans un cercle d'apprentissage d'Amérique centrale pour promouvoir et adapter le modèle d'Abriendo Oportunidades à d'autres populations de filles vulnérables au Costa Rica, au Salvador, en Haiti, au Honduras et au Nicaragua.

Le programme d'autonomisation des adolescentes (PAA)

Mis en œuvre en partenariat avec YWCA Zambie de 2013 à 2016, le programme a atteint plus de 10000 filles vulnérables âgées de 10-19 ans dans cinq sites ruraux répartis dans quatre provinces de Zambie. Les filles ont été séparées en groupes de 10-14 ans, de 15-19 ans et de filles mariées/mères. Le programme s'est efforcé d'améliorer les conditions sociales, sanitaires et économiques des filles, en leur fournissant des compétences clés et des opportunités pour les aider à passer de l'adolescence à l'âge adulte de manière saine. Le PAA était un essai aléatoire et contrôlé en grappe qui testait trois combinaisons d'interventions :
1. Les groupes des espaces sûrs, animés par une jeune mentore locale, dans lesquels les filles se rencontrent une fois par semaine pendant deux ans pour une formation sur la santé sexuelle et reproductive, sur les compétences de la vie courante et sur l'éducation financière.

2. Des espaces sûrs et un bon de santé que les filles peuvent utiliser dans des établissements privés et publics conventionnés pour des services de bienêtre général et de santé sexuelle et reproductive ; et

3. Des espaces sûrs, un bon de santé et un compte d'épargne conçu pour les filles.

Il y avait également un site de contrôle dans lequel les filles ne bénéficiaient d'aucune intervention.

\section{Initiatives pour les adolescentes-Kenya (AGI-K)}

L'AGI-K était un essai randomisé mis en œuvre sur deux sites: dans la zone urbaine de Kibera, un quartier informel de Nairobi ; et dans la zone rurale de Wajir, une région isolée et sous-développée du Kenya, le long de la frontière nord-est avec la Somalie. L'AGI-K a bénéficié à plus de 6000 filles âgées de 11 à 15 ans entre 2015 et 2017, et les travaux de recherche étaient encore en cours au moment de la rédaction du présent document.

L'AGI-K a testé les quatre modules suivants :

1. La prévention de la violence, qui comprend des dialogues communautaires sur la prévalence et la persistance de la violence à l'égard des filles, associés à des projets communautaires financés pour relever ces défis.

2. Prévention de la violence + éducation, qui comprend également un transfert d'argent conditionné à l'inscription à l'école au début de chaque trimestre et à la fréquentation régulière tout au long du trimestre.

3. Prévention de la violence + éducation + santé, où les filles se réunissent une fois par semaine dans des groupes d'espace sûr avec une mentore qui dispense un programme de formation sur la santé, les compétences de la vie courante et la nutrition.

4. Prévention de la violence + éducation + santé + création de richesses, où les filles participent également à des sessions supplémentaires d'éducation financière intégrées dans les programmes de l'espace sûr et ouvrent des comptes d'épargne ou des banques à domicile.

Les résultats intermédiaires de I'AGI-K révèlent des 
impacts positifs pour les filles sur un large éventail d'indicateurs sanitaires, sociaux, éducatifs et financiers, tant à Kibera qu'à Wajir.

- À Kibera, I'AGI-K a eu des effets significatifs sur la réduction de la violence, le taux d'achèvement des études primaires et l'auto-efficacité scolaire, les connaissances en matière de santé sexuelle et reproductive, les dispositifs de sécurité sociale, les connaissances financières et le recours à l'épargne, et la situation économique des ménages.

- À Wajir, I'AGI-K a eu des effets statistiquement significatifs sur la scolarisation dans le primaire, les normes positives en matière de genre et l'autoefficacité, l'éducation financière et le recours à l'épargne.

- Les programmes communautaires AGI-K et l'épargne des filles se poursuivent à ce jour, et le Population Council explore des partenariats possibles pour étendre le programme.

\section{BALIKA (Association bangladeshi pour les compétences essentielles de la vie, les revenus et les connaissances des adolescents)}

Le programme BALIKA était un essai contrôlé et randomisé à quatre volets qui a évalué si trois approches de renforcement des compétences visant à autonomiser les filles peuvent effectivement retarder l'âge du mariage chez les filles âgées de 12 à 18 ans dans certaines régions du sud-ouest du Bangladesh où les taux de mariage des enfants sont les plus élevés. Plus de 9000 filles dans 72 communautés ont participé au programme BALIKA en 2014 et 2015. Les communautés ont été assignées à l'un des trois volets d'intervention :

- Soutien dans le domaine de l'éducation : Les filles ont bénéficié d'un tutorat en mathématiques et en anglais (filles scolarisées) et d'une formation en informatique ou en finances (filles non scolarisées).

- Formation aux compétences essentielles : Les filles ont reçu une formation sur les droits des femmes et la négociation, la pensée critique et la prise de décision.

- Formation aux activités productives : Les filles ont reçu une formation à l'entrepreneuriat, à l'entretien des téléphones portables, à la photographie et aux premiers secours.

- 24 autres communautés ont servi de groupe de contrôle dans le cadre de cette étude : aucun service n'a été fourni dans ces communautés.
Toutes les filles participant au programme BALIKA se sont réunies chaque semaine avec des mentores et des pairs dans des lieux sûrs réservés aux filles, ce qui a permis aux filles de nouer des amitiés, de recevoir une formation sur les nouvelles technologies, d'emprunter des livres et d'acquérir les compétences dont elles ont besoin pour passer de l'adolescence à l'âge adulte.

Les résultats ont montré que les filles qui étaient célibataires au début de l'étude avaient moins de chances d'être mariées à la fin de l'étude dans chaque version étudiée. Par rapport aux filles hors des communautés BALIKA, les filles participant au programme avaient plus de chances d'être scolarisées, d'avoir amélioré leurs compétences en mathématiques et de gagner un revenu. À partir de 2017, le Population Council a établi des partenariats avec le FNUAP, I'UNICEF et le Ministère des affaires féminines et de l'enfance du Bangladesh pour étendre le programme BALIKA à de nouveaux districts au Bangladesh.

\section{Biruh Tesfa (Brillant Avenir)}

Le programme Biruh Tesfa cible les adolescentes non scolarisées des bidonvilles urbains d'Éthiopie, en les mettant en contact avec des mentores adultes et en leur offrant une éducation non formelle et un enseignement sur le VIH et les compétences essentielles, et crée des liens avec les services de santé. Le Population Council a conceptualisé ce programme en se basant sur des recherches menées auprès d'adolescents éthiopiens et collabore avec le Ministère éthiopien de la femme, de I'enfance et de la jeunesse et ses bureaux régionaux à Amhara, Addis-Abeba et Tigray pour le gérer.

Le programme Biruh Tesfa emploie des mentores adultes formées qui vont de maison en maison pour trouver des filles non scolarisées éligibles entre 7 et 24 ans et les inviter à participer. Cette approche permet aux mentores de trouver des filles qui pourraient autrement ne pas être incluses, comme les enfants travailleurs domestiques qui sont en grande partie confinés à la maison, les filles handicapées et les enfants travailleurs du sexe. Une fois qu'elles ont été autorisées à s'inscrire, les filles rencontrent régulièrement les mentores et d'autres filles dans des lieux sûrs, publics et réservés aux filles, mis à disposition par le kebele (administration locale). Lors de ces rencontres, les mentores leur enseignent les bases de l'alphabétisation, les compétences essentielles, les connaissances financières et sur l'épargne, et les sensibilisent au VIH et à la santé génésique.

Une évaluation récente du programme a révélé que les filles de la région où le programme Biruh Tesfa a été mis en œuvre étaient plus de deux fois plus susceptibles de déclarer avoir un soutien social que les filles d'une région de comparaison où le programme Biruh Tesfa n'a pas été 
mis en œuvre. Elles avaient également deux fois plus de chances d'obtenir de bons résultats concernant leurs connaissances sur le VIH, de savoir où obtenir des conseils et des tests volontaires, et de vouloir se faire tester. Une autre évaluation a révélé que parmi les filles qui n'avaient jamais fréquenté l'école formelle et qui vivaient dans la région où le programme Biruh Tesfa opérait, les scores moyens aux tests de lecture et de calcul ont augmenté de manière significative. Des améliorations similaires des résultats aux tests n'ont pas été constatées chez les filles qui n'avaient jamais fréquenté l'école formelle et qui vivaient dans une zone de comparaison.

Le programme Biruh Tesfa a atteint plus de 75000 filles non scolarisées dans 18 villes d'Éthiopie et, en 2019, il sera étendu à 10500 filles supplémentaires.

\section{Filles Eveillées}

Le programme Filles Eveillées est un programme de 30 sessions destiné aux adolescentes migrantes employées de maison dans les villes du Burkina Faso. Il a touché 375 filles âgées de 11 à 19 ans employées dans le service domestique à Bobo-Dioulasso et Ouagadougou entre 2011-2013. Le programme Filles Eveillées visait à accroître les opportunités des travailleuses domestiques et à réduire leur vulnérabilité en les mettant en contact avec des services, en renforçant les compétences essentielles, leurs capacités financières et leurs connaissances en matière de santé (y compris la santé sexuelle et reproductive) et en élargissant leur système de sécurité sociale (y compris en leur donnant la possibilité de rencontrer des pairs et des mentores). Pour atteindre cette population isolée, le programme a eu recours à plusieurs strategies:

- faire du porte-à-porte pour atteindre les filles socialement isolées et les recruter pour le programme ;

- tenir régulièrement de rencontres de groupes de filles pour réduire l'isolement social des filles;

- utiliser les recontres de groupe pour renforcer les compétences et les connaissances des filles en matière de protection, en leur conférant des atouts qui ne peuvent plus leur être enlevés ;

- la mise en relation des filles avec les services sanitaires, financiers et psychosociaux disponibles dans leurs communautés

- $\quad$ organiser des séances de sensibilisation et des visites à domicile dans chaque communauté du programme afin d'obtenir le soutien nécessaire.
Une évaluation du programme, le premier programme de développement des ressources pour les filles domestiques au Burkina Faso, a révélé une augmentation significative du pourcentage de filles qui ont déclaré :

- avoir un compte d'épargne ;

- avoir des d'attitudes soucieuses d'équité entre les sexes (par exemple, ne pas être d'accord avec le fait que les hommes ont le droit de battre leurs femmes);

- disposer d'un solide réseau de sécurité sociale (y compris des amis et des personnes à qui s'adresser pour obtenir des conseils); et

- connaître les méthodes efficaces de planification familiale et savoir où les obtenir.

Le programme Filles Eveillées a fourni des outils en français pour le suivi et le rapportage de routine des mentores, élaborés par les mentores:

- Canevas d'Appréciation des Sessions

- Tableau de Présence des Filles aux Sessions

- Fiche Individuelle des Visites à Domicile

- $\quad$ Fiche de Supervision des Activités des Mentors.

Elles sont disponibles en annexe de https:// www.popcouncil.org/uploads/pdfs/2012PGY_ ProcessEval-FillesEveillees.pdf

Le programme a eu un impact important sur les politiques et programmes. Le Ministère de la protection sociale du Burkina Faso utilise l'approche et les preuves obtenues grâce à ce programme pour élaborer une stratégie visant à mieux répondre aux besoins des adolescents domestiques. Au Niger, le FNUAP utilise cette approche pour atteindre des centaines de milliers de filles.

\section{GirlsRead! (Les filles lisent!)}

En 2016, avec le Forum for African Women Educationalists en Zambie (FAWEZA), une ONG locale qui travaille à l'amélioration de l'égalité des sexes dans l'éducation, et Worldreader, une ONG internationale fondée pour remédier à la pénurie chronique de livres dans les pays en développement ; le Population Council a lancé le programme GirlsRead ! Un programme d'éducation et d'autonomisation qui est en cours d'évaluation par le biais d'un essai contrôlé et randomisé.

GirlsRead! combine trois interventions : 
- groupes d'espaces sûrs pour les filles

- des lecteurs électroniques contenant environ 100 livres, pour la plupart de la fiction écrite par des auteurs africains, et chargés à l'aide de panneaux solaires locaux, et

- l'engagement de la communauté pour améliorer les résultats d'apprentissage et réduire la probabilité que les filles quittent l'école prématurément. Des groupes de filles dirigés par des mentores qui favorisent l'établissement de liens sociaux non familiaux, incitent les filles à réfléchir de manière critique sur les préjugés sexistes et les aident à développer les atouts sociaux et la confiance nécessaires pour progresser vers l'école secondaire.

Afin d'évaluer l'efficacité des interventions pour améliorer les compétences en matière

d'alphabétisation, les résultats à l'examen de fin d'études primaires et encourager la progression vers l'école secondaire, le Population Council mène un essai en grappes randomisé à trois volets sur environ 1200 filles en septième année d'études dans 36 communautés des districts de Lusaka, Ndola et Chingola. Les enquêtes sont menées avant et après I'intervention auprès des adolescentes de la septième année, la dernière année de l'école primaire. Les filles sont réparties au hasard dans 3 groupes d'étude :

- Groupe A : un espace sûr + un lecteur électronique + I'engagement communautaire

- Groupe B : un espace sûr + engagement communautaire

- Groupe C: groupe contrôle

Les trois groupes recevront l'ensemble des interventions au cours de la deuxième phase.

\section{NISITU (Écoutez-moi, progressons ensemble)}

Le programme NISITU est une approche centrée sur les filles qui vise à inclure à la fois les filles et les garçons dans un effort communautaire pour promouvoir des normes égalitaires en matière de genre et réduire la violence sexuelle et basée sur le genre chez les adolescentes et les adolescents. Le programme NISITU inclut les filles et les garçons âgés de 10-19 dans les bidonvilles urbains du Kenya. Le programme teste l'idée que les garçons et les jeunes hommes sont une partie importante de l'écologie dans laquelle vivent les filles, et que tant les filles que les garçons bénéficieront d'une transformation des attentes en matière de genre. Les filles et les garçons sont regroupés en groupes unisexes d'environ 20 à 30 jeunes, sous la direction d'une mentore issu de la communauté. Au cours de rencontres hebdomadaires dans un espace sûr, le programme couvre une variété de sujets liés à la santé et droits sexuels et reproductifs, les normes et le pouvoir liés au genre, et le développement économique. Quelques mois après la création des groupes, les groupes d'hommes et de femmes se réunissent tous les mois pour discuter de ce qu'ils ont appris dans leurs groupes respectifs. Le programme NISITU a recours à une conception quasi-expérimentale dans trois communautés : une dans laquelle les garçons et les filles suivent des programmes, une dans laquelle il n'y a qu'un programme pour les filles et un groupe contrôle.

\section{L'épargne sûre et intelligente}

L'épargne sûre et intelligente est un programme développé par le Population Council pour aider les adolescentes vulnérables à se constituer des ressources, des réseaux de soutien et à faire des économies afin de sortir de leur situation de pauvreté. Le programme combine des réunions de groupe hebdomadaires animées par une femme, l'éducation financière, l'éducation à la santé et aux compétences essentielles, et des comptes d'épargne individuels officiels.

Le programme, qui a été piloté et évalué à Nairobi, au Kenya, et à Kampala, en Ouganda, a permis d'accroître les ressources sociales, sanitaires et économiques des filles. Les filles qui participent à ce programme sont beaucoup plus susceptibles que les autres d'avoir des objectifs financiers et des connaissances financières précises, de connaître le VIH et la santé reproductive, et de discuter de questions financières avec leurs parents. Elles sont également moins susceptibles d'être harcelés sexuellement.

La phase pilote du programme a atteint plus de 12000 filles au Kenya et en Ouganda entre 2008 et 2012. Le programme a ensuite été étendu entre 2012 et 2015 à plus de 8000 filles au Kenya, dont la majorité vivent en dehors de Nairobi. 


\section{ANNEXE 3. LIENS VERS LES CURRICULUMS COMPLETS DES PROGRAMMES INCLUS DANS LA BOÎTE À OUTILS DE LA MENTORE}

Programmation centrée sur les filles

- Outils et ressources sur la programmation centrée sur les filles, https://www.popcouncil.org/girlcentered-program-resources

- Conception de programmes centrés sur les filles : Boîte à outils pour développer, renforcer et étendre les programmes centrés sur les filles (2010), http://www.ungei.org/ files/ 2010PGY_AdolGirlToolkitComplete.pdf

- Les compétences de protection des filles : Une collection d'outils pour la conception de programmes (2016), http:// knowledgecommons.popcouncil.org/ departments_sbsr-pgy/559/

\section{Abriendo Oportunidades}

- Matériel didactique pour les séances de travail (2018): https:// knowledgecommons.popcouncil.org/ departments_sbsr-pgy/620/

- Ouvrir des opportunités pour réaliser mon projet de vie : programme de 5 mois (2016):

- 8 à 13 ans: https://knowledgecommons. popcouncil.org/departments_sbsr-pgy/549/

- 14 à 18 ans: https://knowledgecommons. popcouncil.org/departments_sbsr-pgy/548/

- Programme Abriendo Oportunidades : Guide intégré des curriculums ( 8 à 17 ans) - Cycle de deux ans (2015):

- Anglais : https://knowledgecommons. popcouncil.org/departments_sbsr-pgy/592/

- Espagnol : https://knowledgecommons. popcouncil.org/departments_sbsr-pgy/615/

- Adapté pour le Belize (anglais): https:// knowledgecommons.popcouncil.org/ departments_sbsr-pgy/517/

- Outils pour la formation des mentores: https:/ /knowledgecommons.popcouncil.org/ departments_sbsr-pgy/593/

- Ouvrir des opportunités pour les enfants : https:// knowledgecommons.popcouncil.org/ departments_sbsr-pgy/616/
- Ouvrir des opportunités : session « Plan de vie» (2016):

- Brochure Q'eqchi: https:// knowledgecommons.popcouncil.org/ departments_sbsr-pgy/617/

- Brochure en espagnol: https:// knowledgecommons.popcouncil.org/ departments_sbsr-pgy/618/

- Session «Plan de vie » : https:// knowledgecommons.popcouncil.org/ departments_sbsr-pgy/619/

AGEP

- Programme d'études sur les services de santé adaptés aux adolescents et les mécanismes de bons de santé : Manuel de formation des facilitateurs (2015), https:// knowledgecommons.popcouncil.org/departments_ sbsr-pgy/587/

- Curriculum sur la nutrition pour le programme d'autonomisation des adolescentes (2014), https:// knowledgecommons.popcouncil.org/departments_ sbsr-pgy/586/

- Curriculum sur l'éducation financière pour le programme d'autonomisation des adolescentes (2013), https://

knowledgecommons.popcouncil.org/departments_ sbsr-pgy/585/

- Curriculum sur la santé et sur les compétences essentielles pour le programme d'autonomisation des adolescentes (2013), https:// knowledgecommons.popcouncil.org/ departments_sbsr-pgy/584/

AGI-K

- Initiative pour les adolescentes - Kenya : Curriculum sur la santé et les compétences essentielles à la vie courante, Wajir (2015), https:// knowledgecommons.popcouncil.org/departments_ sbsr-pgy/591/

- Initiative pour les adolescentes - Kenya : Curriculum sur l'éducation financière, Wajir (2015), https:// knowledgecommons.popcouncil.org/ departments_sbsr-pgy/590/ 
- Initiative pour les adolescentes - Kenya :

Curriculum sur la santé et les compétences

essentielles à la vie courante, Kibera (2015), https://

knowledgecommons.popcouncil.org/departments_ sbsr-pgy/589/

- Initiative pour les adolescentes - Kenya :

Curriculum sur l'éducation financière, Kibera

(2015), https://

knowledgecommons.popcouncil.org/departments_ sbsr-pgy/588/

Filles Eveillées

- Filles Eveillées: Programme pour les Adolescentes Employées de Maison-Guide du mentor (2011), https://knowledgecommons.popcouncil.org/ departments_sbsr-pgy/582/

\section{GirlsRead!}

- Girls Rights: An Empowerment Curriculum (2018), https://knowledgecommons.popcouncil.org/ departments_sbsr-pgy/456/

\section{NISITU}

- NISITU (Nisikilize Tujengane): Listen to Me, Let's Grow Together Fact Sheet (2018), https:// knowledgecommons.popcouncil.org/departments_ sbsr-pgy/464/

\section{Safe and Smart Savings}

- Young Women: Your Future, Your MoneyWorkbook for Girls Ages 10-14 in Kenya (2011), https://knowledgecommons.popcouncil.org/ departments_sbsr-pgy/574/

- Young Women: Your Future, Your MoneyWorkbook for Girls Ages 15-19 in Kenya (2011), https:/ /knowledgecommons.popcouncil.org/ departments_sbsr-pgy/575/

- Young Women: Your Future, Your MoneyWorkbook for Girls Ages 10-14 in Uganda (2011), https://knowledgecommons.popcouncil.org/ departments_sbsr-pgy/576/

- Young Women: Your Future, Your MoneyWorkbook for Girls Ages 15-19 in Uganda (2011), https://knowledgecommons.popcouncil.org/ departments_sbsr-pgy/577/ 


\section{RESSOURCES ET LECTURES SUPPLÉMENTAIRES}

Amin, S., J.A. Ahmed, M. Saha, M. Hossain, and E. Haque. 2016. Delaying child marriage through community-based skills-development programs for girls: Results from a randomized controlled study in rural Bangladesh. New York and Dhaka, Bangladesh: Population Council. https://knowledgecommons. popcouncil.org/departments_sbsr-pgy/557/.

Austrian, K., E. Muthengi, A. Wambugu, D. Ghati, and E. Kariuki. 2012. Safe and smart savings for vulnerable girls in Kenya and Uganda: The evolving model, lessons learned, and recommendations. New York: Population Council. https://knowledgecommons.popcouncil.org/ departments_sbsr-pgy/578/.

Jarvis, L. and G. Kabore. 2012. Process evaluation: The Filles Eveillées (Girls Awakened) program for migrant adolescent girls in domestic service in urban Burkina Faso. Ouagadougou: Population Council. https:// knowledgecommons.popcouncil.org/departments_ sbsr-pgy/581/.

Population Council. 2018. NISITU (Nisikilize Tujengane): Listen to Me, Let's Grow Together. https:// knowledgecommons.popcouncil.org/departments_ sbsr-pgy/464/.

Salem, R., B. Ibrahim, and M. Brady. 2003. Negotiating leadership roles: Young women's experience in rural Egypt. Women's Studies Quarterly 31(3/4): 174-191. www.jstor.org/stable/40003326. 


\section{population pomatia \\ Ideas. Evidence. Impact.}

Le Population Council aide à renforcer la capacité de différents acteurs à concevoir, mettre en ouvre et évaluer des programmes efficaces et évolutifs qui vise à améliorer les conditions sanitaires, sociales, économiques et cognitives des adolescentes. Pour télécharger ta série d'outils et de ressources pratiques sur la programmation centrée sur les filles, visitez le site:

popcouncil.org/girl-centered-program-resources

\section{GIRL * Center

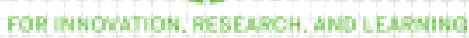

Le Girl Innovation, Research, and Learning (GIRL) Center gënère, synthétise et traduit des données pour transformer la vie des adolescentes.

popcouncil.org/girlcenter 